Chemical and Isotopic Variations

of Precipitation in the Los Alamos Region, New Mexico
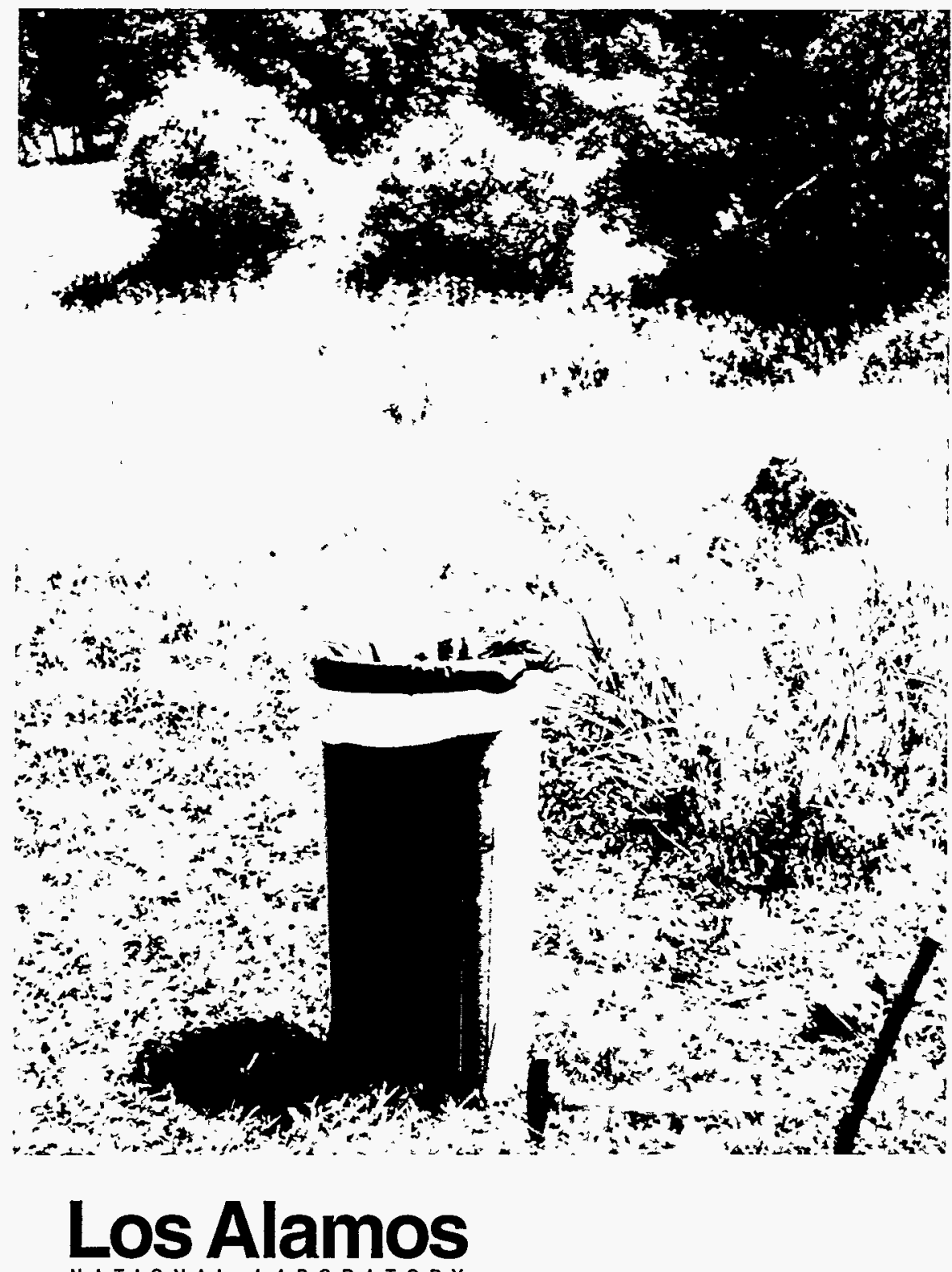

NATIONAL LABORATORY

Los Alamos National Laboratory is operated by the University of California for the United States Department of Energy under contract W-7405-ENG-36. 
Cover photo: Los Alamos National Laboratory photograph of the precipitation collector at the site near VC-2B, Sulphur Springs.

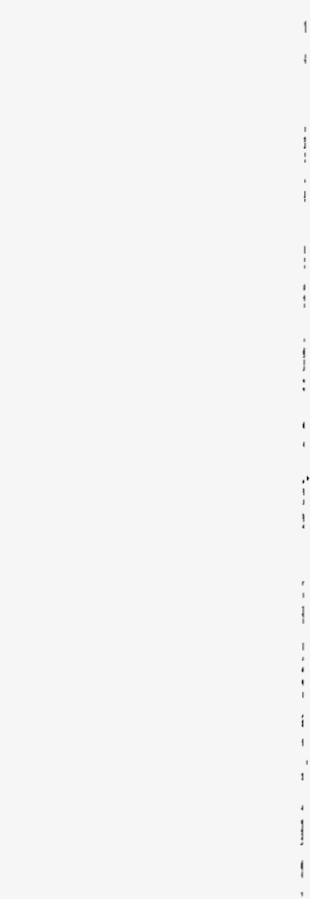

An Affirmative Action/Equal Opportunity Employer

This report was prepared as an account of work sponsored by an agency of the United States Government. Neither The Regents of the University of California, the United States Government nor any agency thereof, nor any of their employees, makes any warranty, express or implied, or assumes any legal liability or responsibility for the accuracy, completeness, or usefulness of any information, apparatus, product, or process disclosed, or represents that its use would not infringe privately owned rights. Reference herein to any specific commercial product, process, or service by trade name, trademark, manufacturer, or otherwise, does not necessarily constitute or imply its endorsement, recommendation, or favoring by The Regents of the University of California, the United States Government, or any agency thereof. The views and opinions of authors expressed herein do not necessarily state or reflect those of The Regents of the University of Californin, the United States Government, or any agency thereof. 
Chemical and Isotopic Variations

of Precipitation in the Los Alamos Region,

New Mexico

Andrew I. Adams

Fraser Goff

Dale Counce 


\section{DISCLAIMER}

Portions of this document may be illegible in electronic image products. Images are produced from the best available original document. 


\title{
CHEMICAL AND ISOTOPIC VARIATIONS OF PRECIPITATION IN THE LOS ALAMOS REGION, NEW MEXICO
}

by

\author{
Andrew I. Adams, Fraser Goff, and Dale Counce
}

\begin{abstract}
Precipitation collectors were installed at 14 locations on the Pajarito Plateau and surrounding areas to study variations in chemistry, stable isotopes and tritium for the years 1990 to 1993 . The volume of precipitation was measured and samples were collected and analyzed every three to four months. All precipitation samples contain $<2.50 \mathrm{mg} / \mathrm{kg} \mathrm{Cl}$ and have $\mathrm{pH}$ values ranging from 5.4 to 6.7 . The stable isotope $\left(\delta \mathrm{D} / \delta^{18} \mathrm{O}\right)$ results record seasonal variations in precipitation as the weather patterns shift from sources in the Pacific Ocean to sources in the Gulf of Mexico. The stable isotope results also show isotopic variations due to elevation differences among the collection points. The tritium contents $\left({ }^{3} \mathrm{H}\right)$ in rain samples vary from 6.54 T.U. to 141 T.U. Contouring of high tritium values (e.g. $>20$ T.U.) from each collection period clearly shows that Laboratory activities release some tritium to the atmosphere. The effect of these releases are well below the limits set by the Environmental Protection Agency for drinking water (about 6200 T.U.). The magnitude of the releases is apparently greatest during the summer months. However, anomalous tritium values are detected as far north as Española, New Mexico for many collection periods. Tritium releases by the Laboratory are not constant; thus, the actual amount of tritium in each release has been diluted in the composite samples of our three to four month collection periods.
\end{abstract}

\section{INTRODUCTION}

In February of 1990 the Geology and Geochemistry group of the Earth and Environmental Sciences Division began a regional study of the background levels of tritium, stable isotopes, and chemistry in precipitation for the Los Alamos region of New Mexico. This investigation is part of the framework studies supporting the Environmental Restoration Program at Los Alamos National Laboratory. The data presented herein include major element, trace element, and isotopic analyses for 155 samples collected from 14 precipitation stations during 1990 to 1993. The major element analyses include 13 elements and the trace element analyses include 28 elements. The isotopic analyses consist of oxygen-18, deuterium, and tritium (the radioactive isotope of hydrogen; half-life $=12.4$ years). This study was not intended to measure the amount of precipitation but we have calculated the approximate amount of precipitation falling at each station by dividing the volume of the water collected by the area of the mouth of the collector (Table 1).

Los Alamos is located on the Pajarito Plateau in north central New Mexico (Fig. 1). The sampling area extends from Española in the north to Santa $F e$ in the south and stretches from the western side of Valles caldera in the Jemez Mountains across the Española Basin to the Santa Fe ski basin in the Sangre de Cristo Range.

\section{FIELD WORK AND ANALYTICAL METHODS}

Precipitation was obtained from 14 sites (Table 1) using collectors modified from the design of White et al. (1990). The collectors are made from large metal trash cans containing collection bags. Large, doubled, plastic trash bags were installed in each collector, and the bags were necked down to less than $10 \mathrm{~mm}$ and taped to prevent evaporation of precipitation after entering the bags. The doubled bags were taped to the rim of the trash can to hold them in place (Figs. 2 and 3). Samples were obtained from the collectors at each site every three to four months. During each collection the precipitated water was emptied from the collector, the volume of water was measured, samples were collected, and the bags replaced. The chemistry samples were analyzed according to procedures described in Trujillo et al. (1987). The stable isotope samples were collected unfiltered in $30 \mathrm{ml}$ glass bottles with polyseal caps and filled brimful leaving no head space. These 
TABLE 1. Field Data for Precipitation Collectors in the Los Alamos Region, New Mexico(a)

\begin{tabular}{|c|c|c|c|c|c|c|}
\hline $\begin{array}{c}\text { Sample } \\
\text { No. }\end{array}$ & Description & $\begin{array}{l}\text { Date } \\
\text { In }\end{array}$ & $\begin{array}{l}\text { Date } \\
\text { Out }\end{array}$ & $\begin{array}{l}\text { Volume (b) } \\
\text { (liters) }\end{array}$ & $\begin{array}{c}\text { Rainfall } \\
(\mathrm{mm})\end{array}$ & $\begin{array}{l}\text { Rainfall } \\
\text { (inches) }\end{array}$ \\
\hline WC-1 & Santa Fe Airport & $2 / 90$ & $05 / 23 / 90$ & 6.5 & 54.39 & 2.14 \\
\hline WC-9 & Santa Fe Airport & $05 / 23 / 90$ & $08 / 09 / 90$ & 7.5 & 62.76 & 2.47 \\
\hline WC-28 & Santa Fe Airport & $08 / 09 / 90$ & $11 / 28 / 90$ & 10 & 83.68 & 3.29 \\
\hline WC-46 & Santa Fe Airport & $11 / 28 / 90$ & $04 / 25 / 91$ & 5.75 & 48.12 & 1.89 \\
\hline WC-51 & Santa Fe Airport & $04 / 25 / 91$ & $07 / 29 / 91$ & 24 & 200.83 & 7.91 \\
\hline WC-67 & Santa Fe Airport & $07 / 29 / 91$ & $12 / 04 / 91$ & 20 & 167.36 & 6.59 \\
\hline WC-80 & Santa Fe Airport & $12 / 04 / 91$ & $04 / 20 / 92$ & 9.5 & 79.50 & 3.13 \\
\hline WC-97 & Santa Fe Airport & $04 / 20 / 92$ & $08 / 14 / 92$ & 15.5 & 129.71 & 5.11 \\
\hline WC-113 & Santa Fe Airport & $08 / 14 / 92$ & $12 / 04 / 92$ & 8.9 & 74.48 & 2.93 \\
\hline WC-146 & Santa Fe Airport & $08 / 04 / 93$ & $11 / 18 / 93$ & 12 & 100.42 & 3.95 \\
\hline WC-2 & Tech Area 33 & $2 / 90$ & $05 / 23 / 90$ & 11.75 & 98.32 & 3.87 \\
\hline WC-8 & Tech Area 33 & $05 / 23 / 90$ & $08 / 09 / 90$ & 14 & 117.15 & 4.61 \\
\hline WC-25 & Tech Area 33 & $08 / 09 / 90$ & $11 / 27 / 90$ & 15 & 125.52 & 4.94 \\
\hline WC-37 & Tech Area 33 & $11 / 27 / 90$ & $04 / 23 / 91$ & 15 & 125.52 & 4.94 \\
\hline WC-54 & Tech Area 33 & $04 / 23 / 91$ & $08 / 01 / 91$ & 20 & 167.32 & 6.59 \\
\hline WC-70 & Tech Area 33 & $08 / 01 / 91$ & $12 / 09 / 91$ & 28.5 & 238.49 & 9.39 \\
\hline WC-83 & Tech Area 33 & $12 / 09 / 91$ & $04 / 20 / 92$ & 12.3 & 102.93 & 4.05 \\
\hline WC-102 & Tech Area 33 & $04 / 20 / 92$ & $08 / 20 / 92$ & 21 & 175.73 & 6.92 \\
\hline WC- 110 & Tech Area 33 & $08 / 20 / 92$ & $12 / 03 / 92$ & 9.1 & 76.15 & 3.00 \\
\hline WC-125 & Tech Area 33 & $12 / 03 / 92$ & $04 / 28 / 93$ & 18 & 150.62 & 5.93 \\
\hline WC-138 & Tech Area 33 & $04 / 28 / 93$ & $08 / 09 / 93$ & 13.5 & 112.97 & 4.45 \\
\hline WC-149 & Tech Area 33 & $08 / 09 / 93$ & $12 / 03 / 93$ & 18 & 150.62 & 5.93 \\
\hline WC-3 & Philomena's & $2 / 90$ & $05 / 23 / 90$ & 7 & 58.58 & 2.31 \\
\hline WC-19 & Philomena's & $05 / 23 / 90$ & $09 / 07 / 90$ & 25 & 209.02 & 8.24 \\
\hline WC-33 & Philomena's & $09 / 07 / 90$ & $12 / 04 / 90$ & 15 & 125.52 & 4.94 \\
\hline WC-42 & Philomena's & $12 / 04 / 90$ & $04 / 24 / 91$ & 11.1 & 92.88 & 3.66 \\
\hline WC-57 & Philomena's & $04 / 24 / 91$ & $08 / 05 / 91$ & 25 & 209.20 & 8.24 \\
\hline WC-77 & Philomena's & $08 / 05 / 91$ & $12 / 19 / 91$ & 26.5 & 221.75 & 8.73 \\
\hline WC-79 & Philomena's & $12 / 19 / 91$ & $03 / 31 / 92$ & $13.5(\mathrm{c})$ & 112.97 & 4.45 \\
\hline WC-99 & Philomena's & $03 / 31 / 92$ & $08 / 14 / 92$ & 20 & 167.63 & 6.59 \\
\hline WC-107 & Philomena's & $08 / 14 / 92$ & $12 / 03 / 92$ & 9.8 & 82.01 & 3.23 \\
\hline WC-127 & Philomena's & $12 / 03 / 92$ & $04 / 29 / 93$ & 16 & 133.89 & 5.27 \\
\hline WC- 140 & Philomena's & $04 / 29 / 93$ & $08 / 13 / 93$ & 10.5 & 87.86 & 3.46 \\
\hline WC-152 & Philomena's & $08 / 13 / 93$ & $11 / 29 / 93$ & 13.75 & 115.06 & 4.53 \\
\hline WC-4 & Pajarito Mountain & $2 / 90$ & $05 / 23 / 90$ & 15 & 125.52 & 4.94 \\
\hline WC-20 & Pajarito Mountain & $05 / 23 / 90$ & $09 / 07 / 90$ & 26.1 & 218.41 & 8.60 \\
\hline WC-34 & Pajarito Mountain & $09 / 07 / 90$ & $12 / 07 / 90$ & 15 & 125.52 & 4.94 \\
\hline WC-45 & Pajarito Mountain & $12 / 07 / 90$ & $04 / 25 / 91$ & 8 & 66.95 & 2.64 \\
\hline WC-61 & Pajarito Mountain & $04 / 25 / 91$ & $08 / 06 / 91$ & 35 & 292.89 & 11.53 \\
\hline WC-69 & Pajarito Mountain & $08 / 06 / 91$ & $12 / 08 / 91$ & 33 & 276.15 & 10.87 \\
\hline WC-90 & Pajarito Mountain & $12 / 08 / 91$ & $05 / 07 / 92$ & 25.5 & 213.39 & 8.40 \\
\hline WC-93 & Pajarito Mountain & $05 / 07 / 92$ & $08 / 10 / 92$ & 28.5 & 238.48 & 9.39 \\
\hline WC-116 & Pajarito Mountain & $08 / 10 / 92$ & $12 / 16 / 92$ & 17.4 & 145.61 & 5.73 \\
\hline WC-130 & Pajarito Mountain & $12 / 16 / 92$ & $05 / 04 / 93$ & 23 & 192.47 & 7.58 \\
\hline WC-136 & Pajarito Mountain & $05 / 04 / 93$ & $08 / 09 / 93$ & 13 & 108.79 & 4.28 \\
\hline WC-155 & Pajarito Mountain & $08 / 09 / 93$ & $02 / 02 / 94$ & 22.5 & 188.28 & 7.41 \\
\hline WC-5 & "S" Site & $2 / 90$ & $05 / 30 / 90$ & 15.1 & 126.36 & 4.97 \\
\hline WC-15 & "S" Site & $05 / 30 / 90$ & $09 / 05 / 90$ & 2 & 16.76 & 0.66 \\
\hline WC-31 & "S" Site & $09 / 05 / 90$ & $12 / 04 / 90$ & 22 & 184.14 & 7.25 \\
\hline WC-41 & "S" Site & $12 / 04 / 90$ & $04 / 24 / 91$ & 14.1 & 117.99 & 4.65 \\
\hline WC-56 & "S" Site & $04 / 24 / 91$ & $08 / 05 / 91$ & 29.5 & 246.86 & 9.72 \\
\hline WC-78 & "S"Site & $08 / 05 / 91$ & $12 / 19 / 91$ & 33 & 276.15 & 10.87 \\
\hline WC-87 & "S" Site & $12 / 19 / 91$ & $04 / 29 / 92$ & 11.5 & 96.23 & 3.79 \\
\hline
\end{tabular}




\begin{tabular}{|c|c|c|c|c|c|c|}
\hline $\begin{array}{c}\text { Sample } \\
\text { No. }\end{array}$ & Description & $\begin{array}{l}\text { Date } \\
\text { In }\end{array}$ & $\begin{array}{l}\text { Date } \\
\text { Out }\end{array}$ & $\begin{array}{c}\text { Volume (b) } \\
\text { (liters) }\end{array}$ & $\begin{array}{l}\text { Rainfall } \\
\text { (mm) }\end{array}$ & $\begin{array}{l}\text { Rainfall } \\
\text { (inches) }\end{array}$ \\
\hline WC-103 & "S" Site & $04 / 29 / 92$ & $08 / 20 / 92$ & 25.5 & 213.39 & 8.40 \\
\hline WC-115 & "S" Site & $08 / 20 / 92$ & $12 / 16 / 92$ & 9.1 & 76.15 & 3.00 \\
\hline WC-129 & "S" Site & $12 / 16 / 92$ & 04/30/93 & 24 & 200.83 & 7.91 \\
\hline WC-134 & "S" Site & $04 / 30 / 93$ & 08/05/93 & 15.5 & 129.71 & 5.11 \\
\hline WC-153 & "S" Site & $08 / 05 / 93$ & $11 / 30 / 93$ & 28 & 238.48 & 9.39 \\
\hline WC-6 & White Rock "Y" & $2 / 90$ & $05 / 30 / 90$ & 7 & 58.58 & 2.31 \\
\hline WC-10 & White Rock "Y" & $05 / 30 / 90$ & 08/09/90 & 19.5 & 163.18 & 6.42 \\
\hline WC-32 & White Rock "Y" & $08 / 09 / 90$ & $12 / 04 / 90$ & 12 & 100.42 & 3.95 \\
\hline WC-43 & White Rock "Y" & $12 / 04 / 90$ & $04 / 24 / 91$ & 11.3 & 94.56 & 3.72 \\
\hline WC-55 & White Rock "Y" & $04 / 24 / 91$ & $08 / 01 / 91$ & 26.25 & 219.66 & 8.65 \\
\hline WC-72 & White Rock "Y" & $08 / 01 / 91$ & $12 / 10 / 91$ & 21 & 175.73 & 6.92 \\
\hline WC-84 & White Rock "Y" & $12 / 10 / 91$ & 04/28/92 & 14.5 & 121.34 & 4.78 \\
\hline WC-98 & White Rock "Y" & $04 / 28 / 92$ & $08 / 14 / 92$ & 15.5 & 129.70 & 5.11 \\
\hline WC-108 & White Rock "Y" & $08 / 14 / 92$ & $12 / 03 / 92$ & 5.8 & 48.53 & 1.91 \\
\hline WC-126 & White Rock "Y" & $12 / 03 / 92$ & $04 / 29 / 93$ & 18.5 & 154.81 & 6.09 \\
\hline WC-137 & White Rock "Y" & 04/29/93 & 08/09/93 & 19 & 158.99 & 6.26 \\
\hline WC-148 & White Rock "Y" & $08 / 09 / 93$ & $11 / 29 / 93$ & 10.5 & 87.86 & 3.46 \\
\hline WC-7 & Espanola Ranger Station & $2 / 90$ & $05 / 30 / 90$ & 5.1 & 42.68 & 1.68 \\
\hline WC-14 & Espanola Ranger Station & $05 / 30 / 90$ & 09/05/90 & 1.75 & 14.64 & 0.58 \\
\hline WC-30 & Espanola Ranger Station & 09/05/90 & $11 / 28 / 90$ & 12 & 100.42 & 3.95 \\
\hline WC-44 & Espanola Ranger Station & $11 / 28 / 90$ & $04 / 24 / 91$ & 6.5 & 54.34 & 2.14 \\
\hline WC-58 & Espanola Ranger Station & $04 / 24 / 91$ & $08 / 05 / 91$ & 15.5 & 129.71 & 5.11 \\
\hline WC-71 & Espanola Ranger Station & 08/05/91 & $12 / 10 / 91$ & 21 & 175.73 & 6.92 \\
\hline WC-86 & Espanola Ranger Station & $12 / 10 / 91$ & 04/29/92 & 10.5 & 87.87 & 3.46 \\
\hline WC-101 & Espanola Ranger Station & 04/29/92 & 08/20/92 & 17 & 142.46 & 5.60 \\
\hline WC-114 & Espanola Ranger Station & $08 / 20 / 92$ & $12 / 16 / 92$ & 8.2 & 68.62 & 2.70 \\
\hline WC-128 & Espanola Ranger Station & $12 / 16 / 92$ & $04 / 30 / 93$ & 11 & 92.05 & 3.62 \\
\hline WC-133 & Espanola Ranger Station & $04 / 30 / 93$ & $08 / 05 / 93$ & 7 & 58.58 & 2.31 \\
\hline WC-147 & Espanola Ranger Station & $08 / 05 / 93$ & $11 / 29 / 93$ & 9 & 75.31 & 2.96 \\
\hline WC-11 & Boundary Peak Area & $07 / 26 / 90$ & 09/04/90 & 7 & 58.58 & 2.31 \\
\hline WC-23 & Boundary Peak Area & 09/04/90 & $11 / 26 / 90$ & 20.5 & 171.55 & 6.75 \\
\hline WC-36 & Boundary Peak Area & $11 / 26 / 90$ & $04 / 23 / 91$ & 4.7 & 39.33 & 1.55 \\
\hline WC-49 & Boundary Peak Area & $04 / 23 / 91$ & 07/29/91 & 19.3 & 161.51 & 6.36 \\
\hline WC-94 & Boundary Peak Area & $05 / 07 / 92$ & $08 / 10 / 92$ & 22.25 & 186.19 & 7.33 \\
\hline WC-124 & Boundary Peak Area & 08/10/92 & $04 / 28 / 93$ & 20 & 167.36 & 6.59 \\
\hline WC-131 & Boundary Peak Area & $04 / 28 / 93$ & 08/03/93 & 4 & 33.47 & 1.32 \\
\hline WC-143 & Boundary Peak Area & 08/03/93 & $11 / 16 / 93$ & 11.75 & 98.33 & 3.87 \\
\hline WC-12 & West of Guaje Mtn. & 07/26/90 & 09/04/90 & 11 & 92.05 & 3.62 \\
\hline WC-24 & West of Guaje Mtn. & 09/04/90 & $11 / 27 / 90$ & 22 & 184.40 & 7.25 \\
\hline WC-39 & West of Guaje Mtn. & $11 / 27 / 90$ & 04/23/91 & 13.5 & 112.97 & 4.45 \\
\hline WC-50 & West of Guaje Mtn. & $04 / 23 / 91$ & $07 / 29 / 91$ & 24 & 200.83 & 7.91 \\
\hline WC-76 & West of Guaje Mtn. & $07 / 29 / 91$ & $12 / 18 / 91$ & 25 & 209.21 & 8.24 \\
\hline WC-85 & West of Guaje Mtn. & $12 / 18 / 91$ & 04/29/92 & 16.5 & 138.08 & 5.44 \\
\hline WC-92 & West of Guaje Mtn. & $04 / 29 / 92$ & $08 / 10 / 92$ & 6 & 50.21 & 1.98 \\
\hline WC-106 & West of Guaje Mtn. & 08/10/92 & 12/02/92 & 12.6 & 105.44 & 4.15 \\
\hline WC-119 & West of Guaje Mtn. & $12 / 02 / 92$ & $04 / 23 / 93$ & 14 & 117.15 & 4.61 \\
\hline WC-135 & West of Guaje Mtn. & $04 / 23 / 93$ & 08/06/93 & 17.25 & 144.35 & 5.68 \\
\hline WC-144 & West of Guaje Mtn. & 08/06/93 & $11 / 17 / 93$ & 23.3 & 194.98 & 7.68 \\
\hline WC-13 & Santa Fe Ski Basin & $05 / 23 / 94$ & 09/05/90 & 15.5 & 129.70 & 5.11 \\
\hline WC-29 & Santa Fe Ski Basin & $09 / 05 / 90$ & $11 / 28 / 90$ & 20 & 167.36 & 6.59 \\
\hline WC-47 & Santa Fe Ski Basin & $11 / 28 / 90$ & $04 / 25 / 91$ & 30 & 251.04 & 9.88 \\
\hline WC-52 & Santa Fe Ski Basin & $04 / 25 / 91$ & $07 / 29 / 91$ & 33 & 276.14 & 10.87 \\
\hline WC-66 & Santa Fe Ski Basin & $07 / 29 / 91$ & $12 / 04 / 91$ & 33.5 & 280.33 & 11.04 \\
\hline
\end{tabular}




\begin{tabular}{|c|c|c|c|c|c|c|}
\hline $\begin{array}{c}\text { Sample } \\
\text { No. }\end{array}$ & Description & $\begin{array}{l}\text { Date } \\
\text { In }\end{array}$ & $\begin{array}{l}\text { Date } \\
\text { Out }\end{array}$ & $\begin{array}{l}\begin{array}{l}\text { Volume (b) } \\
\text { (liters) }\end{array} \\
\text { (lit) }\end{array}$ & $\begin{array}{l}\text { Rainfall } \\
\text { (mm) }\end{array}$ & $\begin{array}{c}\text { Rainfall } \\
\text { (inches) }\end{array}$ \\
\hline WC-81 & Santa Fe Ski Basin & $12 / 04 / 91$ & $04 / 20 / 92$ & 39.5 & 330.54 & 13.01 \\
\hline WC-96 & Santa Fe Ski Basin & $04 / 20 / 92$ & $08 / 14 / 92$ & 31 & 259.41 & 10.21 \\
\hline WC-112 & Santa Fe Ski Basin & $08 / 14 / 92$ & $12 / 04 / 92$ & 20.3 & 169.87 & 6.69 \\
\hline WC-123 & Santa Fe Ski Basin & $12 / 04 / 92$ & $04 / 28 / 93$ & 21 & 175.73 & 6.92 \\
\hline WC-132 & Santa Fe Ski Basin & $04 / 28 / 93$ & $\overline{0} 8 / 04 / 93$ & 14.5 & 121.34 & 4.78 \\
\hline WC-145 & Santa Fe Ski Basin & $08 / 04 / 93$ & $11 / 18 / 93$ & 20 & 167.36 & 6.59 \\
\hline WC-16 & VC-2B, Sulphur Springs & $05 / 24 / 90$ & $09 / 06 / 90$ & 8 & 66.94 & 2.64 \\
\hline WC-21 & VC-2B, Sulphur Springs & $09 / 06 / 90$ & $10 / 24 / 90$ & 11 & 92.05 & 3.62 \\
\hline WC-22 & VC-2B, Sulphur Springs & $10 / 24 / 90$ & $11 / 26 / 90$ & 7 & 58.58 & 2.31 \\
\hline WC-35 & VC-2B, Sulphur Springs & $11 / 26 / 90$ & $04 / 23 / 91$ & 24 & 200.83 & 7.91 \\
\hline WC-48 & VC-2B, Sulphur Springs & $04 / 23 / 91$ & $07 / 29 / 91$ & 18.5 & 154.81 & 6.09 \\
\hline WC-68 & VC-2B, Sulphur Springs & $07 / 29 / 91$ & $12 / 05 / 91$ & 28.5 & 238.49 & 9.39 \\
\hline WC-89 & VC-2B, Sulphur Springs & $12 / 05 / 91$ & $05 / 01 / 92$ & 27.5 & 230.12 & 9.06 \\
\hline WC-105 & VC-2B, Sulphur Springs & $05 / 01 / 92$ & $12 / 02 / 92$ & 12.6 & 105.44 & 4.15 \\
\hline WC-118 & VC-2B, Sulphur Springs & $12 / 02 / 92$ & $04 / 21 / 93$ & 17.5 & 146.44 & 5.77 \\
\hline WC-130a & VC-2B, Sulphur Springs & $04 / 21 / 93$ & 08/03/93 & 9 & 75.31 & 2.97 \\
\hline WC-142 & VC-2B, Sulphur Springs & 08/03/93 & $11 / 16 / 93$ & 21.5 & 179.91 & 7.08 \\
\hline WC-17 & Tech Area 49 & $05 / 24 / 90$ & $09 / 06 / 90$ & 3.5 & 29.29 & 1.15 \\
\hline WC-26 & Tech Area 49 & $09 / 06 / 90$ & $11 / 27 / 90$ & 15 & 125.52 & 4.94 \\
\hline WC-38 & Tech Area 49 & $11 / 27 / 90$ & $04 / 23 / 91$ & 8.75 & 73.22 & 2.88 \\
\hline WC-53 & Tech Area 49 & $04 / 23 / 91$ & $08 / 01 / 91$ & 26 & 217.57 & 8.57 \\
\hline WC-73 & Tech Area 49 & $08 / 01 / 91$ & $12 / 16 / 91$ & 37.5 & 313.80 & 12.35 \\
\hline WC-82 & Tech Area 49 & $12 / 16 / 91$ & $04 / 28 / 92$ & 9.25 & 77.40 & 3.05 \\
\hline WC-95 & Tech Area 49 & $04 / 28 / 92$ & $08 / 10 / 92$ & 20 & 167.36 & 6.59 \\
\hline WC-111 & Tech Area 49 & $08 / 10 / 92$ & $12 / 03 / 92$ & 12.5 & 104.60 & 4.12 \\
\hline WC-121 & Tech Area 49 & $12 / 03 / 92$ & $04 / 23 / 93$ & 13.75 & 115.06 & 4.53 \\
\hline WC-150 & Tech Area 49 & $08 / 09 / 93$ & $11 / 29 / 93$ & 17.5 & 146.44 & 5.77 \\
\hline WC-18 & Tech Area 51 & $05 / 24 / 90$ & $09 / 06 / 90$ & 11.5 & 96.23 & 3.79 \\
\hline WC-27 & Tech Area 51 & $09 / 06 / 90$ & $11 / 27 / 90$ & 16 & 133.89 & 5.27 \\
\hline WC-40 & Tech Area 51 & $11 / 27 / 90$ & $04 / 24 / 91$ & 11.6 & 97.07 & 3.82 \\
\hline WC-59 & Tech Area 51 & $04 / 24 / 91$ & $08 / 05 / 91$ & 34 & 284.51 & 11.20 \\
\hline WC-75 & Tech Area 51 & $08 / 05 / 91$ & $12 / 17 / 91$ & 26.5 & 221.75 & 8.73 \\
\hline WC-88 & Tech Area 51 & $12 / 17 / 91$ & $04 / 29 / 92$ & 11.75 & 98.32 & 3.87 \\
\hline WC-100 & Tech Area 51 & $04 / 29 / 92$ & $08 / 14 / 92$ & 18 & 150.62 & 5.93 \\
\hline WC-109 & Tech Area 51 & $08 / 14 / 92$ & $12 / 03 / 92$ & 12.5 & 104.60 & 4.12 \\
\hline WC-122 & Tech Area 51 & $12 / 03 / 92$ & $04 / 23 / 93$ & 17 & 142.26 & 5.60 \\
\hline WC-139 & Tech Area 51 & $04 / 23 / 93$ & $08 / 13 / 93$ & 19 & 158.99 & 6.26 \\
\hline WC-151 & Tech Area 51 & $08 / 13 / 93$ & $11 / 29 / 93$ & 19.5 & 163.18 & 6.42 \\
\hline WC-60 & Kim Manley's House & $04 / 24 / 91$ & $08 / 05 / 91$ & 24 & 200.83 & 7.91 \\
\hline WC-74 & Kim Manley's House & $08 / 05 / 91$ & $12 / 15 / 91$ & 36.75 & 307.52 & 12.11 \\
\hline WC-91 & Kim Manley's House & $12 / 15 / 91$ & $05 / 27 / 92$ & $13(c)$ & 108.78 & 4.28 \\
\hline WC-104 & Kim Manley's House & $05 / 27 / 92$ & $08 / 20 / 92$ & 23 & 192.46 & 7.58 \\
\hline WC-117 & Kim Manley's House & $08 / 20 / 92$ & $12 / 18 / 92$ & 16.8 & 140.58 & 5.53 \\
\hline WC-120 & Kim Manley's House & $12 / 18 / 92$ & $04 / 23 / 93$ & 13 & 108.78 & 4.28 \\
\hline WC-141 & Kim Manley's House & $04 / 23 / 93$ & $.08 / 13 / 93$ & 13 & 108.78 & 4.28 \\
\hline WC-154 & Kim Manley's House & $08 / 13 / 93$ & $11 / 30 / 93$ & 19 & 158.99 & 6.26 \\
\hline
\end{tabular}

(a) Rainfall is calculated from volume divided by surface area

(b) Volume measurements are of varying accuracy but usually \pm 0.1 liter

(c) Indicates estimated volume 


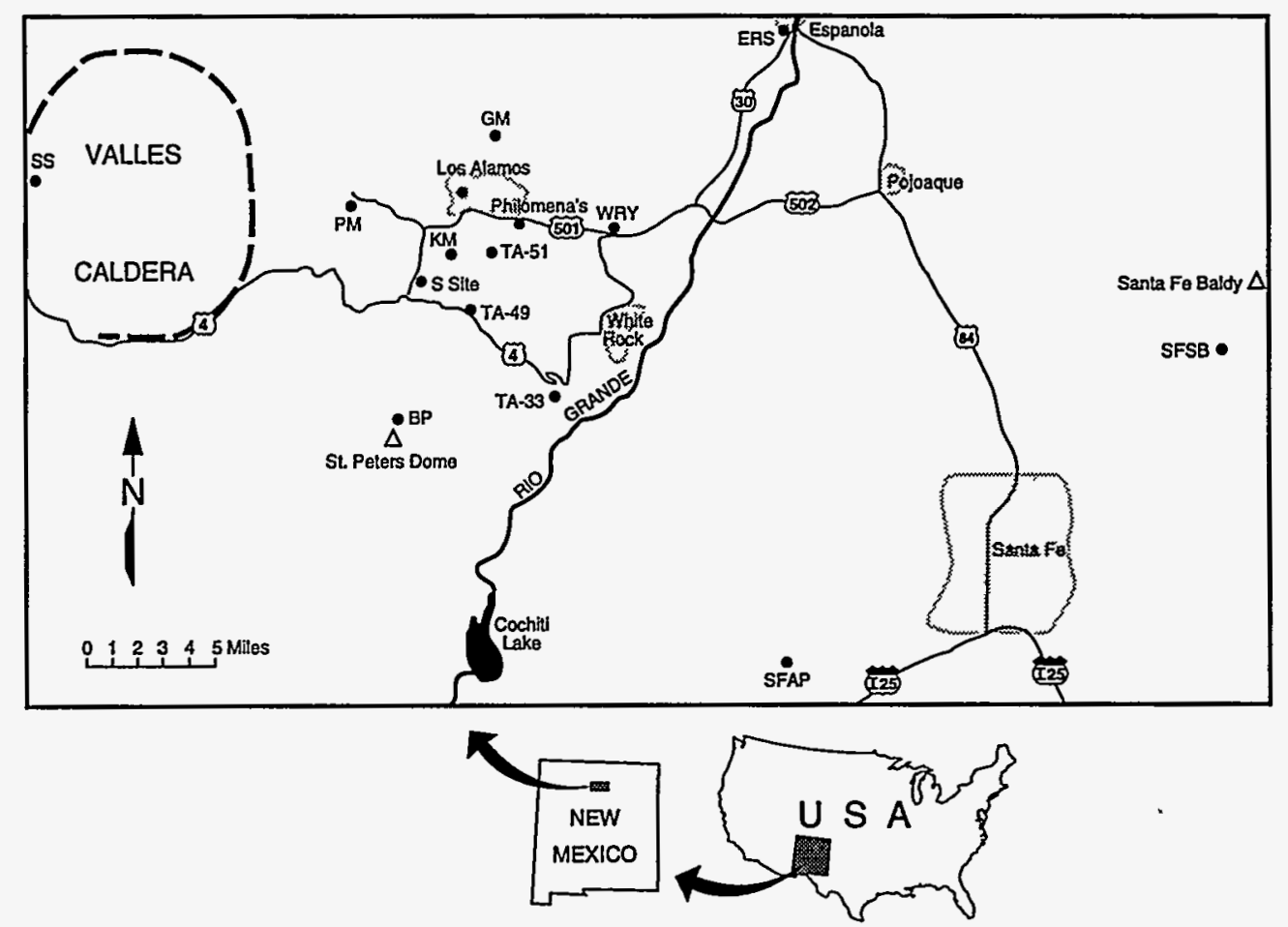

Figure 1: Location map of Los Alamos region, New Mexico showing sites of 14 precipitation collectors; Sites: $G M=$ Guaje Mountain, $K M=$ Kim Manley's, $P M=$ Pajarito Mountain, $B P=$ Boundary Peak, WRY = White Rock "Y", SS = Sulphur Springs, SFAP = Santa Fe airport, SFSB = Santa Fe Ski Basin (see Table 1).

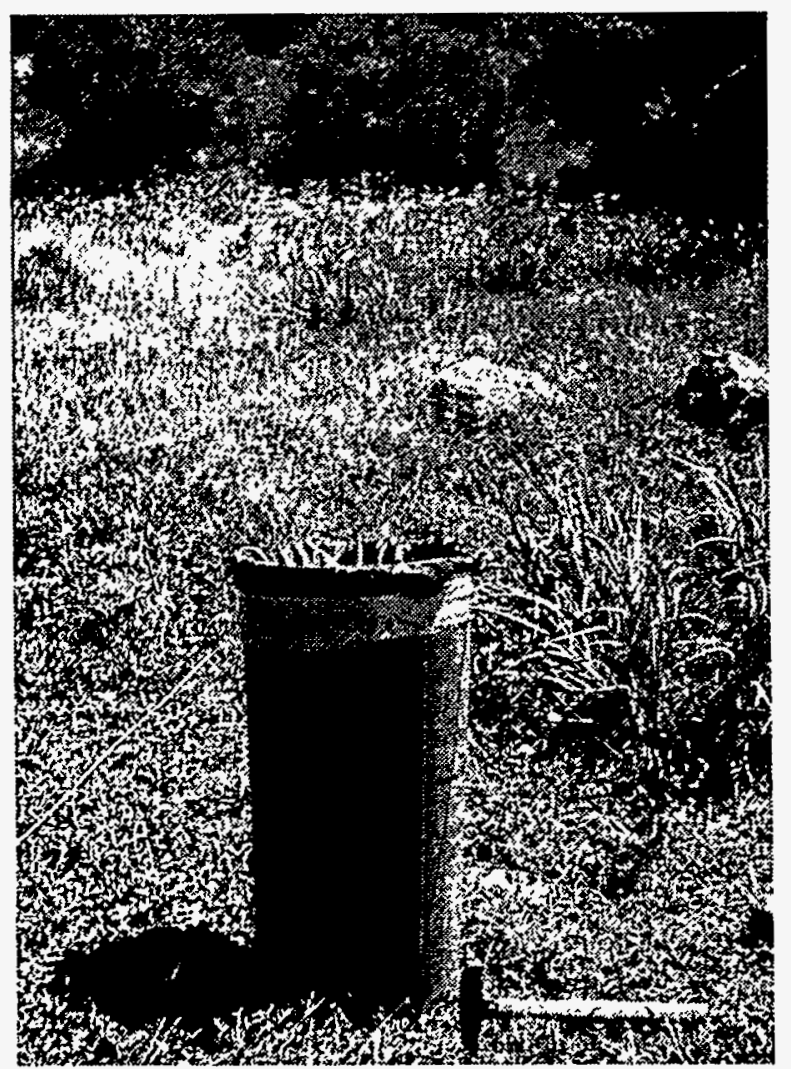

Figure 2: Photograph of the precipitation collector at the site near VC-2B, Sulphur Springs.

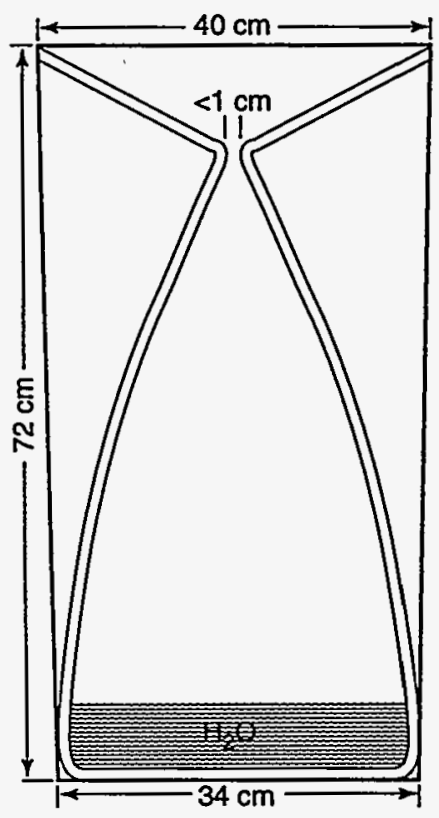

Figure 3: Cutaway diagram of a precipitation collector made from a large trash can, doubled plastic garbage bags, and tape. The bags are necked down with tape wrapped around a pencil to give an entrance diameter of about $1 \mathrm{~cm}$. When the pencil is removed, the plastic expands. Precipitation water (melted snow or rain) can enter but water vapor formed by evaporation primarily condenses inside the bag. 
samples were analyzed by the Stable Isotope Laboratory at Southern Methodist University. Tritium samples were collected in $500 \mathrm{ml}$ plastic bottles with polyseal caps allowing no head space and sent to the Tritium Laboratory at the University of Miami for low level tritium analyses.

Because of difficult weather conditions in winter, some stations were collected only twice a year. Large animals (elk or deer?) created problems at the Boundary Peak site during the dry seasons (late spring and fall) by pushing the collector over and tearing the bags apart to get water. Another problem encountered was occasionally finding trash in the collectors at Santa Fe ski basin and at Pajarito Mountain ski hill. The chemistry of the contaminated samples shows elevated levels of chloride, sodium, and aluminum (Tables 2 and 3; sample WC-45). Contaminated samples were never analyzed for tritium and stable isotopes.

\section{RESULTS AND DISCUSSION}

Chemistry: We found that the major element chemistry of regional precipitation is typical for most precipitation in the United States (Junge and Werby, 1958; Drever, 1982). Tables 2 and 3 list major and trace element chemistry for all samples collected. Chloride concentrations have been corrected (using blanks) for small amounts of chloride adsorbed on the bags during their manufacture. As can be seen in the tables, regional precipitation contains trace amounts of many constitutes and varies from season to season and station to station. When compared to cold groundwaters of the region (Trainer, 1974; Goff and Sayer, 1980; Shevenell et al., 1987; Blake et al., in press), regional precipitation is very dilute. As the moisture in clouds moves away from coastal regions of the continent, the concentrations of sodium and chloride decrease (Junge and Werby, 1958). However, the concentrations we have found are higher than expected for this area. By looking at a plot of sodium versus chloride (Fig. 4), we see a fairly linear trend formed by the data points. The molar ratio of sodium to chloride indicates that these species are contributed from salt in dust, sea salt, or perhaps from sait used on paved roads in the winter months. In contrast, a plot of calcium versus chloride (Fig. 4) shows considerable scatter. In the arid southwest calcium is contributed primarily from dust particles dissolved by precipitation (Junge and Werby, 1958). Sulfate and nitrate are usually indicators of anthropogenic contributions to precipitation (Drever, 1982). As can be seen in Tables 2 and 3 , the sulfate and nitrate levels in regional precipitation are low (usually $<5 \mathrm{mg} / \mathrm{kg}$ sulfate and $<3 \mathrm{mg} / \mathrm{kg}$ nitrate) and indicate little environmental impact (Drever, 1982).

Stable Isotopes: The stable isotopes of hydrogen and oxygen (expressed as $\delta \mathrm{D}$ and $\delta^{18} \mathrm{O}$ in units of per mil) for regional precipitation are listed in Table 4. Most isotope samples plot on or near the world meteoric water line (WMWL; Craig, 1961; Fig. 5) but isotope values vary from season to season and with elevation (Figs. 6 and 7). A few of the sample points plot some distance from the WMWL due probably to storms of unusual isotopic composition or, in some cases, due to evaporation of very small amounts of precipitation in the collectors during dry seasons. The oxygen- 18 values of precipitation at a given station are generally heavier (less depleted) in the summer months and lighter (more depleted) in the winter months. The deuterium values show the same basic trend but are not as pronounced as the oxygen values. The seasonal variations are a result of storm tracks shifting from origins in the Pacific Ocean (winter) to origins in the Gulf of Mexico (summer). Elevation also affects the stable isotope values of precipitation among stations; lighter values at higher elevations and heavier values at lower elevations (Hoefs, 1973).

Tritium: The amount of tritium found in precipitation of the Los Alamos region (Table 4) varies from present background levels of about 10 T.U. (Goff et al., 1985; Shevenell et al., 1987; Shevenell and Goff, in press) to almost two orders of magnitude above expected background levels (Adams and Goff, 1991). By drawing a 20 T.U. (Tritium Units; 1 T.U. = about $3.2 \mathrm{pCi} / \mathrm{kg} \mathrm{H}_{2} \mathrm{O}$ ) contour through the data plotted on maps for different collection periods (Figs. 8-23), the areas where anomalous tritium occurs can be examined. The 20 T.U. contour is always centered over Los Alamos National Laboratory. Stations located inside the Laboratory and near Los Alamos town site show elevated tritium levels in precipitation for almost every collection period. Greater than background levels of tritium occur in precipitation as far north as Española during 1990 and 1991. When correlated with wind directions of the region, we observe that the shape of the 20 T.U. contour is elongated to the northeast in the direction of the prevailing winds (Adams and Goff, 1994). Three stations always show normal background levels of tritium in precipitation (Santa Fe ski basin, Santa Fe airport, and 
TABLE 2. Major Element Chemistry for Precipitation in the Los Alamos Region, New Mexico (a)

\begin{tabular}{|c|c|c|c|c|c|c|c|c|c|c|c|c|c|c|c|c|c|c|c|}
\hline$\underset{\#}{\text { Sample }}$ & Description & Date & $\begin{array}{c}\mathrm{B} \\
\mathrm{mg} / \mathrm{kg}\end{array}$ & $\begin{array}{c}B r \\
m g / k g\end{array}$ & $\begin{array}{c}\mathrm{Ca} \\
\mathrm{mg} / \mathrm{kg}\end{array}$ & $\begin{array}{l}\mathrm{Cl}(\mathrm{b}) \\
\mathrm{mg} / \mathrm{kg}\end{array}$ & $\begin{array}{c}F \\
\mathrm{mg} / \mathrm{kg}\end{array}$ & $\begin{array}{l}\mathrm{HCO3} \\
\mathrm{mgkg}\end{array}$ & $\begin{array}{c}\mathrm{K} \\
\mathrm{mg} / \mathrm{kg}\end{array}$ & $\underset{\mathrm{mg} / \mathrm{kg}}{\mathrm{i}}$ & $\begin{array}{c}\mathrm{Mg} \\
\mathrm{mg} k \mathrm{~kg}\end{array}$ & $\begin{array}{c}\mathrm{Na} \\
\mathrm{mg} / \mathrm{kg}\end{array}$ & $\begin{array}{c}\mathrm{siO2} \\
\mathrm{mg} / \mathrm{kg}\end{array}$ & $\begin{array}{c}\mathrm{SO} 4 \\
\mathrm{mg} / \mathrm{kg}\end{array}$ & $\begin{array}{c}\mathrm{Sr} \\
\mathrm{mg} / \mathrm{kg}\end{array}$ & $\begin{array}{c}\text { TDS } \\
\mathrm{mg} / \mathrm{kg}\end{array}$ & $\begin{array}{l}\text { Cation } \\
\text { Sum }\end{array}$ & $\begin{array}{l}\text { Anion } \\
\text { Sum }\end{array}$ & Balance \\
\hline & $\begin{array}{l}\text { Onnking Waler } \\
\text { Criteria }\end{array}$ & ... & 1 & $\cdots$ & $\ldots$ & & 2 & $240-305$ & $\cdots$ & $\cdots$ & $\ldots$ & $\ldots$ & $\ldots$ & 250 & $\cdots$ & 500 & $\cdots$ & $\cdots$ & $\cdots$ \\
\hline $\begin{array}{l}\text { WC-1 } \\
\text { WC-9 } \\
\text { wC-28 } \\
\text { WC-46 } \\
\text { wC-51 } \\
\text { wC-67 } \\
\text { wC-80 } \\
\text { WC-97 } \\
\text { WC-113 } \\
\text { WC-146 }\end{array}$ & $\begin{array}{l}\text { Santa Fe Airport } \\
\text { Santa Fe Airport } \\
\text { Santa Fe Aimport } \\
\text { Santa Fe Airport } \\
\text { Santa Fe Airport } \\
\text { Santa Fe Airport } \\
\text { Santa Fe Airport } \\
\text { Santa Fe Airport } \\
\text { Santa Fe Airport } \\
\text { Santa Fe Airport }\end{array}$ & $\begin{array}{l}05 / 2390 \\
080990 \\
11 / 2890 \\
042591 \\
07 / 2991 \\
120491 \\
042091 \\
08 / 1492 \\
120492 \\
11 / 1899\end{array}$ & $\begin{array}{l}<0.05 \\
<0.05 \\
<0.02 \\
<0.02 \\
<0.05 \\
<0.05 \\
<0.05 \\
<0.05 \\
<0.01 \\
<0.02\end{array}$ & $\begin{array}{l}<0.05 \\
<0.05 \\
<0.02 \\
<0.01 \\
<0.01 \\
<0.01 \\
<0.02 \\
<0.02 \\
<0.02 \\
0.03\end{array}$ & $\begin{array}{l}\cdots \\
\ldots . . \\
1.60 \\
1.40 \\
1.70 \\
230 \\
2.30 \\
1.88 \\
2.00\end{array}$ & $\begin{array}{l}0.00 \\
1.16 \\
1.41 \\
0.01 \\
0.04 \\
5.15 \\
1.53 \\
0.00 \\
0.71 \\
0.49\end{array}$ & $\begin{array}{l}<0.02 \\
<0.02 \\
<0.02 \\
<0.02 \\
<0.01 \\
0.18 \\
<0.02 \\
<0.02 \\
<0.02 \\
<0.01\end{array}$ & $\begin{array}{r}1 . .5 \\
19.5 \\
13.4 \\
28.1 \\
8.5 \\
28.1 \\
36.4 \\
1.5 \\
39.4 \\
3.9\end{array}$ & $\begin{array}{l}0.30 \\
0.90 \\
1.16 \\
1.70 \\
0.40 \\
3.90 \\
280 \\
0.90 \\
2.90 \\
5.00\end{array}$ & $\begin{array}{l}\cdots \\
<0.01 \\
<0.01 \\
<0.01 \\
0.02 \\
<0.01 \\
<0.01 \\
<0.01 \\
<0.01\end{array}$ & $\begin{array}{l}\ldots \\
\ldots .28 \\
0.31 \\
0.11 \\
0.35 \\
0.33 \\
0.18 \\
0.20 \\
0.37\end{array}$ & $\begin{array}{r}1.40 \\
1.30 \\
1.50 \\
0.53 \\
0.40 \\
13.40 \\
1.75 \\
0.50 \\
1.05 \\
0.35\end{array}$ & $\begin{array}{l}\ldots . \\
<0.20 \\
<0.20 \\
<0.20 \\
<0.20 \\
<0.20 \\
0.20 \\
0.32 \\
0.17\end{array}$ & $\begin{array}{l}1.14 \\
2.37 \\
2.90 \\
1.99 \\
1.59 \\
5.61 \\
3.46 \\
1.89 \\
3.17 \\
1.83\end{array}$ & $\begin{array}{c}\ldots . \\
0.01 \\
0.01 \\
<0.01 \\
0.00 \\
0.01 \\
0.02 \\
<0.01 \\
0.02\end{array}$ & $\begin{array}{l}\ldots \\
\ldots . . \\
28.4 \\
41.8 \\
14.2 \\
61.3 \\
58.2 \\
16.6 \\
77.6 \\
24.2\end{array}$ & $\begin{array}{c}\ldots \\
\ldots . . \\
0.388 \\
0.349 \\
0.122 \\
0.813 \\
0.715 \\
0.239 \\
1.479 \\
0.454\end{array}$ & $\begin{array}{l}\ldots \\
\ldots . \\
0.383 \\
0.624 \\
0.204 \\
0.789 \\
0.749 \\
0.212 \\
0.921 \\
0.262\end{array}$ & $\begin{array}{r}\ldots \\
\ldots . . \\
0.0116 \\
-0.5653 \\
-0.5022 \\
0.0900 \\
-0.0460 \\
0.1224 \\
0.4644 \\
0.5364\end{array}$ \\
\hline $\begin{array}{l}\text { WC-2 } \\
\text { WC-8 } \\
\text { WC-25 } \\
\text { WC-37 } \\
\text { WC-54 } \\
\text { WC-70 } \\
\text { WC-83 } \\
\text { WC-102 } \\
\text { WC-110 } \\
\text { WC-125 } \\
\text { WC-138 } \\
\text { WC-149 }\end{array}$ & $\begin{array}{l}\text { Tech Area } 33 \\
\text { Tech Area } 33 \\
\text { Tech Area } 33 \\
\text { Tech Area } 33 \\
\text { Tech Area } 33 \\
\text { Tech Area } 33 \\
\text { Tech Area } 33 \\
\text { Tech Area } 33 \\
\text { Tech Area } 33 \\
\text { Tech Area } 33 \\
\text { Tech Area } 33 \\
\text { Tech Area } 33\end{array}$ & $\begin{array}{l}05 / 23190 \\
0809190 \\
11 / 2790 \\
0423191 \\
080191 \\
1209191 \\
04 / 20192 \\
0820192 \\
120392 \\
042893 \\
0809193 \\
1203193\end{array}$ & $\begin{array}{l}<0.05 \\
0.19 \\
<0.02 \\
<0.02 \\
<0.05 \\
<0.05 \\
<0.05 \\
<0.05 \\
<0.01 \\
<0.01 \\
<0.01 \\
<0.02\end{array}$ & $\begin{array}{l}<0.05 \\
<0.05 \\
<0.02 \\
<0.01 \\
<0.01 \\
<0.01 \\
<0.02 \\
<0.02 \\
<0.02 \\
<0.02 \\
<0.01 \\
<0.01\end{array}$ & $\begin{array}{l}\ldots \\
\ldots . . \\
0.50 \\
0.50 \\
1.10 \\
1.00 \\
0.70 \\
1.10 \\
0.80 \\
0.32 \\
1.69 \\
0.60\end{array}$ & $\begin{array}{l}0.26 \\
3.30 \\
0.07 \\
0.18 \\
-0.10 \\
0.21 \\
-0.30 \\
0.10 \\
-0.30 \\
0.05 \\
0.09 \\
0.00\end{array}$ & $\begin{array}{l}<0.02 \\
<0.02 \\
<0.02 \\
<0.02 \\
<0.01 \\
<0.01 \\
<0.02 \\
<0.02 \\
<0.02 \\
<0.02 \\
<0.01 \\
<0.01\end{array}$ & $\begin{array}{r}1 . . \\
18.3 \\
13.4 \\
20.7 \\
7.3 \\
2.6 \\
<0.5 \\
1.7 \\
3.5 \\
<0.8 \\
1.1 \\
<1.0\end{array}$ & $\begin{array}{l}0.70 \\
1.00 \\
0.40 \\
0.40 \\
0.10 \\
0.50 \\
0.70 \\
0.40 \\
0.70 \\
0.20 \\
0.60 \\
0.50\end{array}$ & $\begin{array}{l}\ldots . . \\
<0.01 \\
<0.01 \\
<0.01 \\
<0.01 \\
<0.01 \\
<0.01 \\
<0.01 \\
0.01 \\
<0.01 \\
<0.01\end{array}$ & $\begin{array}{l}\ldots \\
\ldots . .11 \\
0.10 \\
0.11 \\
0.03 \\
0.06 \\
0.08 \\
0.09 \\
0.02 \\
0.15 \\
0.05\end{array}$ & $\begin{array}{l}2.60 \\
2.40 \\
0.42 \\
0.69 \\
0.30 \\
0.60 \\
0.34 \\
0.52 \\
0.18 \\
0.50 \\
0.53 \\
0.08\end{array}$ & $\begin{array}{l}\ldots . \\
\ldots . .20 \\
<0.20 \\
<0.20 \\
<0.20 \\
<0.20 \\
<0.20 \\
<0.20 \\
0.17 \\
0.09 \\
<0.02 \\
0.15\end{array}$ & $\begin{array}{l}1.48 \\
1.26 \\
0.99 \\
0.82 \\
1.78 \\
0.69 \\
0.98 \\
1.18 \\
1.13 \\
0.93 \\
1.94 \\
0.79\end{array}$ & $\begin{array}{l}\ldots . . \\
<0.01 \\
<0.01 \\
<0.01 \\
<0.01 \\
<0.01 \\
0.03 \\
<0.01 \\
<0.01 \\
<0.01 \\
<0.01\end{array}$ & $\begin{array}{r}\ldots . . \\
17.6 \\
25.1 \\
12.2 \\
6.9 \\
4.0 \\
7.2 \\
9.3 \\
3.6 \\
9.3 \\
2.5\end{array}$ & $\begin{array}{l}0.084 \\
0.085 \\
0.099 \\
0.098 \\
0.084 \\
0.121 \\
0.156 \\
0.062 \\
0.178 \\
0.064\end{array}$ & $\begin{array}{c}\ldots \\
\ldots . . \\
0.269 \\
0.390 \\
0.180 \\
0.087 \\
0.040 \\
0.085 \\
0.108 \\
0.045 \\
0.106 \\
0.020\end{array}$ & $\begin{array}{c}\ldots . . \\
-1.0449 \\
-1.2856 \\
-0.5764 \\
0.1248 \\
0.7123 \\
0.3526 \\
0.3658 \\
0.3134 \\
0.5025 \\
1.0464\end{array}$ \\
\hline $\begin{array}{l}\text { WC-3 } \\
\text { WC-19 } \\
\text { WC-33 } \\
\text { WC-42 } \\
\text { WC-57 } \\
\text { WC-77 } \\
\text { WC-79 } \\
\text { WC-99 } \\
\text { WC-107 } \\
\text { WC-127 } \\
\text { WC-140 } \\
\text { WC-152 }\end{array}$ & $\begin{array}{l}\text { Philomena's } \\
\text { Philomena's } \\
\text { Philomena's } \\
\text { Philomena's } \\
\text { Philomena's } \\
\text { Philomena's } \\
\text { Philomena's } \\
\text { Philomena's } \\
\text { Philomenan's } \\
\text { Philomena's } \\
\text { Philomena's } \\
\text { Philomena's }\end{array}$ & 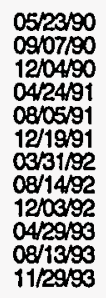 & $\begin{array}{l}<0.05 \\
<0.02 \\
<0.02 \\
<0.02 \\
<0.05 \\
<0.05 \\
<0.05 \\
<0.05 \\
0.01 \\
<0.01 \\
<0.01 \\
<0.02\end{array}$ & $\begin{array}{l}<0.05 \\
<0.05 \\
<0.02 \\
<0.01 \\
<0.01 \\
<0.01 \\
<0.02 \\
<0.02 \\
<0.02 \\
<0.02 \\
<0.01 \\
<0.01\end{array}$ & $\begin{array}{l}\ldots . . \\
0.70 \\
0.40 \\
4.50 \\
0.50 \\
2.30 \\
1.50 \\
0.94 \\
0.89 \\
1.84 \\
0.65\end{array}$ & $\begin{array}{l}0.69 \\
0.00 \\
0.00 \\
0.61 \\
0.27 \\
0.00 \\
3.19 \\
0.00 \\
0.00 \\
0.35 \\
0.14 \\
0.00\end{array}$ & $\begin{array}{l}<0.02 \\
<0.02 \\
<0.02 \\
<0.02 \\
<0.01 \\
<0.01 \\
0.05 \\
<0.02 \\
<0.02 \\
<0.02 \\
<0.01 \\
<0.01\end{array}$ & \begin{tabular}{r|}
19.5 \\
14.6 \\
18.3 \\
8.5 \\
$<0.5$ \\
18.0 \\
1.5 \\
$<0.5$ \\
$<0.8$ \\
3.4 \\
3.4 \\
$<1.0$
\end{tabular} & $\begin{array}{l}0.60 \\
0.20 \\
0.40 \\
0.10 \\
0.60 \\
0.60 \\
0.90 \\
0.60 \\
0.10 \\
0.20 \\
0.60 \\
0.20\end{array}$ & $\begin{array}{l}\cdots \\
<0.01 \\
<0.01 \\
<0.01 \\
<0.01 \\
<0.01 \\
<0.01 \\
<0.01 \\
<0.01 \\
<0.01 \\
<0.01\end{array}$ & $\begin{array}{l}\ldots \\
\ldots . . \\
0.16 \\
0.03 \\
0.15 \\
0.01 \\
0.10 \\
0.09 \\
0.03 \\
0.02 \\
0.17 \\
0.04\end{array}$ & $\begin{array}{l}2.30 \\
0.20 \\
0.38 \\
1.06 \\
0.50 \\
0.60 \\
3.02 \\
0.37 \\
0.11 \\
0.89 \\
0.52 \\
0.18\end{array}$ & $\begin{array}{l}\ldots \\
\ldots 0.20 \\
<0.20 \\
<0.20 \\
<0.20 \\
<0.20 \\
<0.20 \\
0.17 \\
<0.02 \\
<0.02 \\
0.15\end{array}$ & $\begin{array}{l}2.06 \\
1.06 \\
0.95 \\
0.77 \\
1.19 \\
0.89 \\
7.37 \\
1.50 \\
1.00 \\
1.08 \\
1.84 \\
0.87\end{array}$ & $\begin{array}{l}\ldots \ldots \\
<0.01 \\
<0.01 \\
<0.01 \\
<0.01 \\
0.01 \\
<0.01 \\
<0.01 \\
<0.01 \\
<0.01 \\
<0.01\end{array}$ & $\begin{array}{r}\ldots \ldots \\
19.0 \\
19.9 \\
17.1 \\
4.0 \\
37.5 \\
8.1 \\
3.7 \\
5.1 \\
13.3 \\
2.4\end{array}$ & $\begin{array}{l}0.106 \\
0.080 \\
0.289 \\
0.077 \\
0.308 \\
0.159 \\
0.074 \\
0.107 \\
0.238 \\
0.055\end{array}$ & $\begin{array}{l}\ldots . \\
\ldots .284 \\
0.362 \\
0.196 \\
0.044 \\
0.580 \\
0.087 \\
0.040 \\
0.059 \\
0.157 \\
0.025\end{array}$ & $\begin{array}{c}\ldots \\
\ldots . . \\
-0.9091 \\
-1.2761 \\
0.3838 \\
0.5433 \\
-0.6130 \\
0.5848 \\
0.5858 \\
0.5817 \\
0.4072 \\
0.7544\end{array}$ \\
\hline $\begin{array}{l}\text { WC-4 } \\
\text { WC-20 } \\
\text { WC-34 } \\
\text { WC-45 } \\
\text { WC-61 } \\
\text { WC-69 } \\
\text { WC-90 } \\
\text { WC-93 } \\
\text { WC-116 } \\
\text { WC-130 } \\
\text { WC-136 } \\
\text { WC-155 }\end{array}$ & $\begin{array}{l}\text { Pajarito Mountain } \\
\text { Pajarito Mountain } \\
\text { Pajarito Mountain } \\
\text { Pajarito Mountain } \\
\text { Pajanito Mountain } \\
\text { Pajarito Mountain } \\
\text { Pajarito Mountain } \\
\text { Pajarito Mountain } \\
\text { Pajarito Mountain } \\
\text { Pajanto Mountain } \\
\text { Pajarito Mountain } \\
\text { Pajanito Mountain }\end{array}$ & 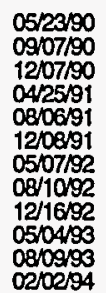 & $\begin{array}{l}<0.05 \\
<0.02 \\
0.02 \\
<0.02 \\
<0.05 \\
<0.05 \\
<0.05 \\
<0.05 \\
<0.01 \\
<0.01 \\
0.01 \\
0.02\end{array}$ & $\begin{array}{l}<0.05 \\
<0.05 \\
<0.02 \\
0.02 \\
<0.01 \\
<0.01 \\
<0.02 \\
<0.02 \\
<0.02 \\
<0.02 \\
<0.01 \\
<0.01\end{array}$ & $\begin{array}{l}\ldots \\
\ldots .70 \\
2.70 \\
0.00 \\
0.60 \\
0.30 \\
1.10 \\
0.77 \\
0.55 \\
1.49 \\
1.90\end{array}$ & $\begin{array}{r}0.00 \\
0.15 \\
1.97 \\
31.70 \\
0.12 \\
0.22 \\
0.00 \\
0.00 \\
0.00 \\
0.00 \\
0.21 \\
2.59\end{array}$ & $\begin{array}{l}<0.02 \\
<0.02 \\
<0.02 \\
0.05 \\
<0.01 \\
<0.01 \\
<0.02 \\
<0.02 \\
<0.02 \\
<0.02 \\
<0.01 \\
0.03\end{array}$ & $\begin{array}{r}20.7 \\
20.7 \\
24.4 \\
26.8 \\
12.2 \\
3.6 \\
<0.5 \\
23 \\
<0.5 \\
<0.8 \\
4.0 \\
2.6\end{array}$ & $\begin{array}{r}0.80 \\
1.10 \\
11.12 \\
10.00 \\
0.60 \\
0.40 \\
0.40 \\
0.40 \\
0.50 \\
0.20 \\
0.90 \\
1.10\end{array}$ & $\begin{array}{l}\ldots . . \\
<0.01 \\
<0.01 \\
<0.01 \\
<0.01 \\
<0.01 \\
<0.01 \\
<0.01 \\
<0.01 \\
<0.01 \\
<0.01\end{array}$ & $\begin{array}{l}\ldots \\
\ldots . . \\
0.67 \\
0.91 \\
0.11 \\
<0.01 \\
0.07 \\
0.08 \\
0.07 \\
0.04 \\
0.19 \\
0.22\end{array}$ & $\begin{array}{r}200 \\
0.40 \\
0.94 \\
21.60 \\
0.20 \\
0.60 \\
0.25 \\
0.36 \\
0.25 \\
0.32 \\
0.62 \\
2.07\end{array}$ & $\begin{array}{c}\ldots . \\
\ldots 0.20 \\
0.20 \\
<0.20 \\
<0.20 \\
<0.20 \\
<0.20 \\
0.15 \\
<0.02 \\
<0.02 \\
<0.20\end{array}$ & $\begin{array}{l}0.93 \\
1.17 \\
5.21 \\
2.73 \\
1.35 \\
0.54 \\
0.85 \\
1.17 \\
0.87 \\
1.23 \\
1.71 \\
2.58\end{array}$ & $\begin{array}{l}\ldots . . \\
<0.01 \\
<0.01 \\
<0.01 \\
<0.01 \\
<0.01 \\
<0.01 \\
<0.01 \\
<0.01 \\
<0.01 \\
0.04\end{array}$ & $\begin{array}{r}\ldots . . \\
49.4 \\
98.3 \\
17.7 \\
7.6 \\
29 \\
7.4 \\
4.2 \\
3.9 \\
14.0 \\
15.9\end{array}$ & $\begin{array}{l}0.541 \\
1.466 \\
0.155 \\
0.111 \\
0.059 \\
0.124 \\
0.096 \\
0.071 \\
0.259 \\
0.267\end{array}$ & $\begin{array}{c}\ldots \\
\ldots . \\
0.634 \\
1.408 \\
0.269 \\
0.095 \\
0.030 \\
0.087 \\
0.039 \\
0.049 \\
0.169 \\
0.211\end{array}$ & $\begin{array}{c}\ldots \\
\ldots . . \\
-0.1577 \\
0.0408 \\
-0.5394 \\
0.1560 \\
0.6358 \\
0.3526 \\
0.8432 \\
0.3703 \\
0.4214 \\
0.2310\end{array}$ \\
\hline
\end{tabular}




\begin{tabular}{|c|c|c|c|c|c|c|c|c|c|c|c|c|c|c|c|c|c|c|c|}
\hline$\underset{\#}{\text { Sample }}$ & Description & Date & $\begin{array}{c}\mathrm{B} \\
\mathrm{mg} / \mathrm{kg}\end{array}$ & $\begin{array}{c}\mathrm{Br} \\
\mathrm{mg} / \mathrm{kg}\end{array}$ & $\begin{array}{c}\mathrm{Ca} \\
\mathrm{mg} / \mathrm{kg}\end{array}$ & $\begin{array}{l}\mathrm{Cl}(\mathrm{b}) \\
\mathrm{mghkg}\end{array}$ & $\begin{array}{c}F \\
\text { mgkg }\end{array}$ & $\begin{array}{l}\mathrm{HCOS} \\
\mathrm{mg} / \mathrm{kg}\end{array}$ & $\begin{array}{c}\mathrm{K} \\
\mathrm{mg} / \mathrm{kg}\end{array}$ & $\begin{array}{c}4 \\
\text { mgkg }\end{array}$ & $\begin{array}{c}\mathrm{Mg} \\
\mathrm{mgkg}\end{array}$ & $\begin{array}{c}\mathrm{Na} \\
\mathrm{mg} / \mathrm{kg}\end{array}$ & $\begin{array}{c}\mathrm{SiO2} \\
\mathrm{mg} / \mathrm{kg}\end{array}$ & $\begin{array}{c}\mathrm{SO}_{4} \\
\mathrm{mgkg}\end{array}$ & $\begin{array}{c}\mathrm{Sr} \\
\mathrm{mg} / \mathrm{kg}\end{array}$ & $\begin{array}{c}\text { TDS } \\
\mathrm{mg} / \mathrm{kg}\end{array}$ & $\begin{array}{l}\text { Cation } \\
\text { Sum }\end{array}$ & $\begin{array}{l}\text { Anion } \\
\text { Sum }\end{array}$ & Balance \\
\hline $\begin{array}{l}\text { WC-5 } \\
\text { WC-15 } \\
\text { WC-31 } \\
\text { WC-41 } \\
\text { WC-56 } \\
\text { WC-78 } \\
\text { WC-87 } \\
\text { WC-103 } \\
\text { WC-115 } \\
\text { WC-129 } \\
\text { WC-134 } \\
\text { WC-153 }\end{array}$ & $\begin{array}{l}\text { "S"Site } \\
\text { "S" Site } \\
\text { "S" Site } \\
\text { "S" Site } \\
\text { "S" Site } \\
\text { "S" Site } \\
\text { "S" Site } \\
\text { "S" Site } \\
\text { "S" Site } \\
\text { "S" Site } \\
\text { "S" Site } \\
\text { "S" Site }\end{array}$ & 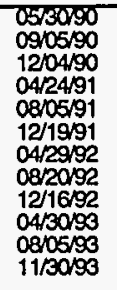 & $\begin{array}{l}<0.05 \\
<0.02 \\
<0.02 \\
<0.02 \\
<0.05 \\
<0.05 \\
<0.05 \\
<0.05 \\
<0.01 \\
<0.01 \\
0.01 \\
<0.02\end{array}$ & $\begin{array}{l}<0.05 \\
<0.05 \\
<0.02 \\
<0.01 \\
<0.01 \\
<0.01 \\
<0.02 \\
<0.02 \\
<0.02 \\
<0.02 \\
<0.01 \\
<0.01\end{array}$ & $\begin{array}{l}\ldots . \\
0.80 \\
0.40 \\
1.20 \\
0.40 \\
0.90 \\
1.20 \\
0.29 \\
0.45 \\
1.20 \\
1.76\end{array}$ & $\begin{array}{l}0.24 \\
2.06 \\
0.00 \\
0.00 \\
0.00 \\
0.00 \\
0.53 \\
0.00 \\
0.00 \\
0.00 \\
0.16 \\
0.42\end{array}$ & $\begin{array}{l}<0.02 \\
0.04 \\
<<.02 \\
<0.02 \\
<<.01 \\
<0.01 \\
<0.02 \\
<0.02 \\
<0.02 \\
<0.02 \\
<0.01 \\
<0.01\end{array}$ & $\begin{array}{r}25.6 \\
19.5 \\
13.4 \\
19.5 \\
7.3 \\
<0.5 \\
1.0 \\
1.2 \\
<0.5 \\
<0.8 \\
<0.8 \\
4.2\end{array}$ & $\begin{array}{l}0.60 \\
4.50 \\
0.30 \\
0.10 \\
0.30 \\
0.30 \\
0.70 \\
0.50 \\
0.10 \\
0.10 \\
0.30 \\
0.40\end{array}$ & $\begin{array}{l}\ldots \ldots \\
\ldots 0.01 \\
<0.01 \\
<0.01 \\
<0.01 \\
<0.01 \\
<0.01 \\
<0.01 \\
0.01 \\
<0.01 \\
<0.01\end{array}$ & $\begin{array}{c}\ldots \\
\cdots . .18 \\
0.05 \\
0.05 \\
0.11 \\
<0.01 \\
0.04 \\
0.06 \\
<0.01 \\
0.01 \\
0.09 \\
0.06\end{array}$ & $\begin{array}{l}0.80 \\
1.60 \\
0.30 \\
0.46 \\
0.30 \\
1.40 \\
1.09 \\
0.48 \\
0.14 \\
0.36 \\
0.53 \\
0.68\end{array}$ & $\begin{array}{l}>-. \\
<0.20 \\
<0.20 \\
<0.20 \\
<0.20 \\
<0.20 \\
<0.20 \\
0.11 \\
<0.02 \\
<0.02 \\
0.11\end{array}$ & $\begin{array}{l}2.20 \\
120 \\
1.64 \\
0.65 \\
1.18 \\
0.75 \\
1.29 \\
1.17 \\
0.20 \\
0.78 \\
1.42 \\
1.03\end{array}$ & $\begin{array}{l}\cdots \\
<0.01 \\
<0.01 \\
<0.01 \\
<0.01 \\
<0.01 \\
0.03 \\
<0.01 \\
<0.01 \\
<0.01 \\
0.01\end{array}$ & $\begin{array}{r}\ldots . \\
\ldots . . \\
19.9 \\
22.7 \\
11.6 \\
4.3 \\
7.6 \\
6.3 \\
1.4 \\
3.2 \\
6.2 \\
10.5\end{array}$ & $\begin{array}{l}0.159 \\
0.056 \\
0.103 \\
0.105 \\
0.136 \\
0.114 \\
0.037 \\
0.061 \\
0.130 \\
0.159\end{array}$ & $\begin{array}{l}\ldots \ldots \\
0.029 \\
0.359 \\
0.165 \\
0.099 \\
0.090 \\
0.070 \\
0.011 \\
0.039 \\
0.073 \\
0.130\end{array}$ & $\begin{array}{c}0 . . \\
-0.5920 \\
-1.4574 \\
-0.4569 \\
0.9134 \\
0.4099 \\
0.4776 \\
1.0755 \\
0.4455 \\
0.5642 \\
0.1985\end{array}$ \\
\hline $\begin{array}{l}\text { WC-6 } \\
\text { WC-10 } \\
\text { WC-32 } \\
\text { WC-43 } \\
\text { WC-55 } \\
\text { WC-72 } \\
\text { WC-84 } \\
\text { WC-98 } \\
\text { WC-108 } \\
\text { WC-126 } \\
\text { WC-137 } \\
\text { WC-148 }\end{array}$ & $\begin{array}{l}\text { White Rock "Y" } \\
\text { White Rock "Y" } \\
\text { White Rock "Y" } \\
\text { White Rock "Y" } \\
\text { White Rock "Y" } \\
\text { White Rock "Y" } \\
\text { White Rock "Y" } \\
\text { White Rock "Y" } \\
\text { White Rock "Y" } \\
\text { White Rock Y" } \\
\text { White Rock "Y" } \\
\text { White Rock "Y" }\end{array}$ & $\begin{array}{l}05 / 3090 \\
080990 \\
120490 \\
04 / 2491 \\
0810191 \\
1211091 \\
04128992 \\
08 / 14192 \\
120392 \\
04 / 29193 \\
0810999 \\
11 / 29193\end{array}$ & $\begin{array}{l}<0.05 \\
<0.05 \\
0.03 \\
<0.02 \\
<0.05 \\
<0.05 \\
<0.05 \\
<0.05 \\
0.01 \\
<0.01 \\
<0.01 \\
<0.02\end{array}$ & $\begin{array}{l}<0.05 \\
<0.05 \\
<0.02 \\
<0.01 \\
<0.01 \\
<0.01 \\
<0.02 \\
<0.02 \\
<0.02 \\
<0.02 \\
<0.01 \\
<0.01\end{array}$ & $\begin{array}{l}\cdots \\
\cdots . . \\
2.50 \\
0.60 \\
0.80 \\
0.30 \\
0.80 \\
1.80 \\
0.98 \\
0.37 \\
1.94 \\
0.69\end{array}$ & $\begin{array}{l}1.55 \\
2.02 \\
2.00 \\
1.09 \\
0.00 \\
0.00 \\
1.02 \\
0.63 \\
0.02 \\
0.61 \\
0.00 \\
0.00\end{array}$ & $\begin{array}{l}<0.02 \\
<0.02 \\
<0.02 \\
<0.02 \\
<0.01 \\
<0.01 \\
<0.02 \\
<0.02 \\
<0.02 \\
<0.02 \\
<0.01 \\
<0.01\end{array}$ & $\begin{array}{r}19.5 \\
18.3 \\
14.6 \\
19.5 \\
6.1 \\
<0.5 \\
<0.5 \\
5.0 \\
<0.5 \\
<0.8 \\
<0.8 \\
<1.0\end{array}$ & $\begin{array}{l}0.30 \\
0.60 \\
1.84 \\
0.10 \\
0.10 \\
0.20 \\
0.70 \\
0.90 \\
0.30 \\
0.20 \\
0.50 \\
0.30\end{array}$ & $\begin{array}{l}\cdots \\
<0.01 \\
<0.01 \\
<0.01 \\
<0.01 \\
<0.01 \\
0.06 \\
0.01 \\
<0.01 \\
<0.01 \\
<0.01\end{array}$ & $\begin{array}{l}\cdots \\
\cdots . .54 \\
0.07 \\
0.07 \\
<0.01 \\
0.05 \\
0.16 \\
0.07 \\
0.01 \\
0.17 \\
0.05\end{array}$ & $\begin{array}{l}2.00 \\
1.60 \\
1.82 \\
1.33 \\
0.30 \\
0.30 \\
1.45 \\
1.17 \\
0.45 \\
0.91 \\
0.40 \\
0.21\end{array}$ & $\begin{array}{l}\cdots \\
<0.20 \\
<0.20 \\
<0.20 \\
<0.20 \\
<0.20 \\
<0.20 \\
0.19 \\
0.02 \\
<0.02 \\
0.09\end{array}$ & $\begin{array}{l}1.91 \\
1.01 \\
6.06 \\
0.89 \\
1.15 \\
0.48 \\
1.23 \\
2.02 \\
1.53 \\
0.90 \\
2.08 \\
1.01\end{array}$ & $\begin{array}{l}\cdots \\
<0.01 \\
<0.01 \\
<0.01 \\
<0.01 \\
<0.01 \\
0.01 \\
<0.01 \\
<0.01 \\
<0.01 \\
<0.01\end{array}$ & $\begin{array}{r}\ldots . . \\
36.9 \\
25.2 \\
9.6 \\
22 \\
6.9 \\
14.5 \\
5.4 \\
4.5 \\
8.3 \\
3.8\end{array}$ & $\begin{array}{l}0.395 \\
0.107 \\
0.074 \\
0.042 \\
0.145 \\
0.240 \\
0.100 \\
0.079 \\
0.166 \\
0.074\end{array}$ & $\begin{array}{c}\ldots . \\
0.535 \\
0.397 \\
0.142 \\
0.027 \\
0.069 \\
0.177 \\
0.063 \\
0.061 \\
0.094 \\
0.043\end{array}$ & $\begin{array}{c}\ldots \\
\ldots . . \\
-0.3001 \\
-1.1499 \\
-0.6329 \\
0.4532 \\
0.5452 \\
0.3039 \\
0.4487 \\
0.2597 \\
0.5559 \\
0.5275\end{array}$ \\
\hline $\begin{array}{l}\text { WC-7 } \\
\text { WC-14 } \\
\text { WC-30 } \\
\text { WC-44 } \\
\text { WC-58 } \\
\text { WC-71 } \\
\text { WC-86 } \\
\text { WC-101 } \\
\text { WC-114 } \\
\text { WC-128 } \\
\text { WC-133 } \\
\text { WC-147 }\end{array}$ & $\begin{array}{l}\text { Espanola Ringer Stn. } \\
\text { Espanola Rnger Stn. } \\
\text { Espanola Rnger Stn. } \\
\text { Espanola Rnger Stn. } \\
\text { Espanola Rnger Stn. } \\
\text { Espanola Rnger Stn. } \\
\text { Espanola Rnger Stn. } \\
\text { Espanola Rnger Stn. } \\
\text { Espanola Rnger Stn. } \\
\text { Espanola Rnger Stn. } \\
\text { Espanola Rnger Stn. } \\
\text { Espanola Rnger Stn. }\end{array}$ & $\begin{array}{l}05 / 20190 \\
09105 / 90 \\
11 / 28 / 90 \\
04 / 24 / 91 \\
08 / 05 / 91 \\
12 / 1091 \\
04 / 2992 \\
08 / 2092 \\
12 / 16 / 92 \\
04 / 30 / 93 \\
08 / 105 / 93 \\
11 / 29 / 93\end{array}$ & $\begin{array}{l}<0.05 \\
<0.02 \\
<0.02 \\
<0.02 \\
<0.05 \\
<0.05 \\
<0.05 \\
<0.05 \\
<0.01 \\
<0.01 \\
0.02 \\
<0.02\end{array}$ & $\begin{array}{l}<0.05 \\
<0.05 \\
<0.02 \\
<0.01 \\
<0.01 \\
<0.01 \\
<0.02 \\
<0.02 \\
<0.02 \\
<0.02 \\
<0.01 \\
<0.01\end{array}$ & $\begin{array}{l}\cdots \\
7 . . \\
1.30 \\
3.10 \\
2.40 \\
0.90 \\
2.00 \\
2.80 \\
1.55 \\
1.75 \\
3.94 \\
1.11\end{array}$ & $\begin{array}{l}0.19 \\
0.00 \\
0.00 \\
0.52 \\
0.05 \\
0.00 \\
0.63 \\
0.00 \\
0.00 \\
0.27 \\
0.61 \\
0.00\end{array}$ & $\begin{array}{l}<0.02 \\
<0.02 \\
<0.02 \\
0.02 \\
<0.01 \\
<0.01 \\
<0.02 \\
<0.02 \\
<0.02 \\
<0.02 \\
<0.01 \\
<0.01\end{array}$ & $\begin{array}{r}20.4 \\
18.3 \\
14.6 \\
23.2 \\
11.0 \\
2.3 \\
5.2 \\
5.2 \\
3.3 \\
<0.8 \\
11.7 \\
<1\end{array}$ & $\begin{array}{l}1.50 \\
0.60 \\
0.24 \\
0.60 \\
0.70 \\
0.20 \\
0.90 \\
0.70 \\
0.40 \\
0.40 \\
1.70 \\
0.20\end{array}$ & $\begin{array}{l}\cdots \\
<0.01 \\
<0.01 \\
<0.01 \\
<0.01 \\
0.01 \\
<0.01 \\
<0.01 \\
<0.01 \\
<0.01 \\
<0.01\end{array}$ & $\begin{array}{c}\ldots \\
\ldots .17 \\
0.21 \\
0.15 \\
<0.01 \\
0.05 \\
0.17 \\
0.05 \\
0.09 \\
0.29 \\
0.06\end{array}$ & $\begin{array}{l}0.80 \\
0.90 \\
0.33 \\
1.03 \\
0.30 \\
0.40 \\
1.15 \\
0.57 \\
0.24 \\
0.68 \\
0.83 \\
0.28\end{array}$ & $\begin{array}{c}\ldots . \\
\ldots 0.20 \\
<0.20 \\
<0.20 \\
<0.20 \\
<0.20 \\
<0.20 \\
0.24 \\
0.19 \\
<0.02 \\
0.15\end{array}$ & $\begin{array}{l}1.94 \\
1.38 \\
0.92 \\
1.75 \\
1.65 \\
0.81 \\
1.40 \\
1.58 \\
0.80 \\
1.31 \\
3.12 \\
0.65\end{array}$ & $\begin{array}{r}\cdots \\
<0.01 \\
<0.01 \\
0.02 \\
<0.01 \\
0.01 \\
0.07 \\
0.01 \\
<0.01 \\
<0.01 \\
<0.01\end{array}$ & $\begin{array}{r}\ldots . \\
19.1 \\
33.5 \\
18.0 \\
6.0 \\
13.5 \\
13.8 \\
8.2 \\
6.9 \\
38.1 \\
3.6\end{array}$ & $\begin{array}{l}0.139 \\
0.247 \\
0.190 \\
0.078 \\
0.209 \\
0.238 \\
0.128 \\
0.152 \\
0.657 \\
0.100\end{array}$ & $\begin{array}{c}\ldots \\
0.286 \\
0.483 \\
0.249 \\
0.078 \\
0.166 \\
0.157 \\
0.093 \\
0.073 \\
0.491 \\
0.030\end{array}$ & $\begin{array}{c}\ldots \\
\ldots . . \\
-0.6905 \\
-0.6455 \\
-0.2720 \\
0.0016 \\
0.1991 \\
0.4125 \\
0.3170 \\
0.7056 \\
0.2893 \\
1.0818\end{array}$ \\
\hline $\begin{array}{l}\text { WC-11 } \\
\text { WC-23 } \\
\text { WC-36 } \\
\text { WC-49 } \\
\text { WC.94 } \\
\text { WC-124 } \\
\text { WC-131 } \\
\text { WC-143 }\end{array}$ & $\begin{array}{l}\text { Boundary Peak Area } \\
\text { Boundary Peak Area } \\
\text { Boundary Peak Area } \\
\text { Boundary Peak Area } \\
\text { Boundary Peak Area } \\
\text { Boundary Peak Area } \\
\text { Boundary Peak Area } \\
\text { Boundary Peak Area }\end{array}$ & $\begin{array}{l}09 / 04 / 90 \\
11 / 26 / 90 \\
04 / 23191 \\
07 / 29191 \\
08 / 1092 \\
04 / 28 / 93 \\
0810393 \\
11 / 16193\end{array}$ & $\begin{array}{l}<0.02 \\
<0.02 \\
<0.02 \\
<0.05 \\
<0.05 \\
0.22 \\
0.02 \\
<0.02\end{array}$ & $\begin{array}{l}<0.05 \\
<0.02 \\
<0.01 \\
<0.01 \\
<0.02 \\
<0.02 \\
<0.01 \\
<0.01\end{array}$ & $\begin{array}{l}\ldots . \\
0.40 \\
0.20 \\
1.20 \\
1.30 \\
0.33 \\
2.74 \\
0.53\end{array}$ & $\begin{array}{l}1.18 \\
0.03 \\
0.00 \\
0.00 \\
0.00 \\
0.00 \\
0.79 \\
0.00\end{array}$ & $\begin{array}{l}<0.02 \\
<0.02 \\
<0.02 \\
<0.01 \\
<0.02 \\
<0.02 \\
<0.01 \\
<0.01\end{array}$ & $\begin{array}{r}22.0 \\
14.6 \\
19.5 \\
6.1 \\
1.9 \\
<0.8 \\
8.8 \\
<1.0\end{array}$ & $\begin{array}{l}0.90 \\
0.50 \\
0.30 \\
0.80 \\
0.60 \\
0.20 \\
3.60 \\
0.20\end{array}$ & $\begin{array}{l}\quad \ldots \\
<0.01 \\
<0.01 \\
<0.01 \\
<0.01 \\
0.03 \\
<0.01 \\
<0.01\end{array}$ & $\begin{array}{l}\ldots . . \\
0.09 \\
0.03 \\
0.14 \\
0.09 \\
0.05 \\
0.42 \\
0.04\end{array}$ & $\begin{array}{l}1.40 \\
0.44 \\
0.33 \\
0.30 \\
0.37 \\
0.34 \\
1.12 \\
0.17\end{array}$ & $\begin{array}{l}<0.20 \\
<0.20 \\
<0.20 \\
<0.20 \\
0.28 \\
<0.02 \\
0.13\end{array}$ & $\begin{array}{l}1.03 \\
0.95 \\
0.37 \\
1.87 \\
1.13 \\
1.10 \\
3.23 \\
0.75\end{array}$ & $\begin{array}{l}<0.01 \\
<0.01 \\
<0.01 \\
<0.01 \\
0.02 \\
<0.01 \\
<0.01\end{array}$ & $\begin{array}{r}18.4 \\
21.6 \\
14.0 \\
7.1 \\
4.0 \\
27.3 \\
3.2\end{array}$ & $\begin{array}{l}0.071 \\
0.040 \\
0.131 \\
0.129 \\
0.071 \\
0.442 \\
0.059\end{array}$ & $\begin{array}{l}0.286 \\
0.342 \\
0.206 \\
0.077 \\
0.052 \\
0.342 \\
0.035\end{array}$ & $\begin{array}{r}-1.2066 \\
-1.5831 \\
-0.4492 \\
0.5055 \\
0.3042 \\
0.2550 \\
0.5221\end{array}$ \\
\hline $\begin{array}{l}\text { WC-12 } \\
\text { wC-24 } \\
\text { wC-39 } \\
\text { WC-50 } \\
\text { wC-76 } \\
\text { WC-85 } \\
\text { WC-92 } \\
\text { WC-106 } \\
\text { WC-119 } \\
\text { WC-135 } \\
\text { WC-144 }\end{array}$ & $\begin{array}{l}\text { West of Guaje Mtn. } \\
\text { West of Guaje Mtn. } \\
\text { West of Guaje Mtn. } \\
\text { West of Guaje Mtn. } \\
\text { West of Guaje Mtn. } \\
\text { West of Guaje Mtn. } \\
\text { West of Guaje Mtn. } \\
\text { West of Guaje Mtn. } \\
\text { West of Guaje Mtn. } \\
\text { West of Guaje Mtn. } \\
\text { West of Guaje Mtn. }\end{array}$ & $\begin{array}{l}09104900 \\
11 / 2790 \\
04 / 2391 \\
07 / 2991 \\
12 / 1891 \\
04129992 \\
08110992 \\
120292 \\
0412393 \\
080693 \\
11 / 1793\end{array}$ & $\begin{array}{l}<0.02 \\
<0.02 \\
<0.02 \\
<0.05 \\
<0.05 \\
<0.05 \\
0.08 \\
0.02 \\
<0.01 \\
0.01 \\
<0.02\end{array}$ & $\begin{array}{l}<0.05 \\
<0.02 \\
<0.01 \\
<0.01 \\
<0.01 \\
<0.02 \\
<0.02 \\
<0.02 \\
<0.02 \\
<0.01 \\
<0.01\end{array}$ & $\begin{array}{l}\ldots . \\
0.40 \\
0.40 \\
0.90 \\
0.80 \\
0.50 \\
2.60 \\
2.18 \\
0.45 \\
120 \\
0.51\end{array}$ & $\begin{array}{l}0.00 \\
0.00 \\
0.36 \\
0.00 \\
0.07 \\
0.00 \\
0.44 \\
0.00 \\
0.00 \\
0.26 \\
0.32\end{array}$ & $\begin{array}{l}<0.02 \\
<0.02 \\
<0.02 \\
<0.01 \\
<0.01 \\
<0.02 \\
<0.02 \\
<0.02 \\
<0.02 \\
<0.01 \\
<0.01\end{array}$ & $\begin{array}{r}19.5 \\
14.6 \\
19.5 \\
8.5 \\
<0.5 \\
<0.5 \\
11.9 \\
1.8 \\
<0.8 \\
11.5 \\
<1.0\end{array}$ & $\begin{array}{l}0.10 \\
0.42 \\
0.50 \\
0.30 \\
0.50 \\
0.70 \\
1.30 \\
0.50 \\
0.20 \\
0.70 \\
0.50\end{array}$ & $\begin{array}{l}<0.01 \\
<0.01 \\
<0.01 \\
<0.01 \\
<0.01 \\
<0.01 \\
<0.01 \\
0.01 \\
<0.01 \\
<0.01\end{array}$ & $\begin{array}{l}\ldots . . \\
0.11 \\
0.08 \\
0.09 \\
0.03 \\
0.06 \\
0.19 \\
0.08 \\
0.05 \\
0.17 \\
0.06\end{array}$ & $\begin{array}{l}0.20 \\
0.28 \\
0.93 \\
0.20 \\
1.30 \\
0.57 \\
0.95 \\
0.29 \\
0.20 \\
0.73 \\
0.60\end{array}$ & $\begin{array}{r}<0.20 \\
<0.20 \\
<0.20 \\
<0.20 \\
<0.20 \\
<0.20 \\
0.19 \\
0.24 \\
<0.02 \\
0.17\end{array}$ & $\begin{array}{l}0.71 \\
0.86 \\
0.74 \\
1.42 \\
1.18 \\
1.04 \\
5.47 \\
1.19 \\
1.01 \\
1.82 \\
0.63\end{array}$ & $\begin{array}{l}<0.01 \\
<0.01 \\
<0.01 \\
<0.01 \\
<0.01 \\
0.05 \\
0.01 \\
0.02 \\
<0.01 \\
<0.01\end{array}$ & $\begin{array}{r}17.9 \\
23.9 \\
12.6 \\
5.6 \\
4.3 \\
26.4 \\
7.9 \\
3.2 \\
9.3 \\
4.3\end{array}$ & $\begin{array}{l}0.058 \\
0.089 \\
0.081 \\
0.123 \\
0.036 \\
0.364 \\
0.149 \\
0.056 \\
0.170 \\
0.009\end{array}$ & $\begin{array}{l}\ldots . . \\
0.280 \\
0.371 \\
0.188 \\
0.057 \\
0.046 \\
0.353 \\
0.084 \\
0.037 \\
0.112 \\
0.046\end{array}$ & $\begin{array}{r}-1.3094 \\
-1.2267 \\
-0.8002 \\
0.7310 \\
0.6022 \\
0.0024 \\
0.5539 \\
0.4156 \\
0.4111 \\
0.6289\end{array}$ \\
\hline
\end{tabular}




\begin{tabular}{|c|c|c|c|c|c|c|c|c|c|c|c|c|c|c|c|c|c|c|c|}
\hline$\underset{\#}{\text { Sample }}$ & Description & Date & $\begin{array}{c}B \\
m g / k g\end{array}$ & $\begin{array}{c}\mathrm{Br} \\
\mathrm{mg} / \mathrm{kg}\end{array}$ & $\begin{array}{c}\mathrm{Ca} \\
\mathrm{mg} / \mathrm{kg}\end{array}$ & $\begin{array}{c}\mathrm{Cl}(\mathrm{b}) \\
\mathrm{mg} / \mathrm{kg}\end{array}$ & $\begin{array}{c}\mathrm{F} \\
\mathrm{mg} / \mathrm{kg}\end{array}$ & $\begin{array}{l}\mathrm{HCOS} \\
\mathrm{mg} / \mathrm{kg}\end{array}$ & $\begin{array}{c}\mathrm{K} \\
\mathrm{mg} / \mathrm{kg}\end{array}$ & $\begin{array}{c}\mathrm{L} \\
\mathrm{mg} / \mathrm{kg}\end{array}$ & $\begin{array}{c}\mathrm{Mg} \\
\mathrm{mg} k \mathrm{~kg}\end{array}$ & $\begin{array}{c}\mathrm{Na} \\
\mathrm{mg} / \mathrm{kg}\end{array}$ & $\begin{array}{c}\mathrm{SiO2} \\
\mathrm{mg} / \mathrm{kg}\end{array}$ & $\begin{array}{c}\mathrm{SO} 4 \\
\mathrm{mg} / \mathrm{kg}\end{array}$ & $\begin{array}{c}\mathrm{Sr} \\
\mathrm{mg} / \mathrm{kg}\end{array}$ & $\begin{array}{c}\text { TDS } \\
\mathrm{mg} / \mathrm{kg}\end{array}$ & $\begin{array}{l}\text { Cation } \\
\text { Sum }\end{array}$ & $\begin{array}{l}\text { Anion } \\
\text { Sum }\end{array}$ & Belance \\
\hline $\begin{array}{l}\text { WC-13 } \\
\text { WC-29 } \\
\text { WC-47 } \\
\text { WC-52 } \\
\text { WC-66 } \\
\text { WC-81 } \\
\text { WC-96 } \\
\text { WC-112 } \\
\text { WC-123 } \\
\text { WC-132 } \\
\text { WC-145 }\end{array}$ & $\begin{array}{l}\text { Santa Fe Skj Basin } \\
\text { Santa Fe Sk Basin } \\
\text { Santa Fe Ski Basin } \\
\text { Santa Fe Sk Basin } \\
\text { Santa Fe Sk Basin } \\
\text { Santa Fe Ski Basin } \\
\text { Santa Fe Sk Basin } \\
\text { Santa Fe Ski Basin } \\
\text { Santa Fe Skj Basin } \\
\text { Santa Fe Sk Basin } \\
\text { Santa Fe Ski Basin }\end{array}$ & $\begin{array}{l}09905190 \\
11 / 2890 \\
04259191 \\
072991 \\
12049191 \\
0420992 \\
081 / 492 \\
120492 \\
042892 \\
080493 \\
11 / 1893\end{array}$ & $\begin{array}{l}<0.02 \\
<0.02 \\
<0.02 \\
<0.05 \\
<0.05 \\
<0.05 \\
<0.05 \\
0.02 \\
<0.01 \\
0.03 \\
<0.02\end{array}$ & $\begin{array}{l}<0.05 \\
<0.02 \\
<0.01 \\
<0.01 \\
<0.01 \\
<0.02 \\
<0.02 \\
<0.02 \\
<0.02 \\
<0.01 \\
<0.01\end{array}$ & $\begin{array}{l}1 . . \\
1.40 \\
0.50 \\
0.70 \\
2.10 \\
0.50 \\
1.00 \\
1.62 \\
0.79 \\
1.05 \\
0.67\end{array}$ & $\begin{array}{l}0.00 \\
0.38 \\
0.00 \\
0.00 \\
0.19 \\
0.00 \\
0.00 \\
0.00 \\
0.48 \\
0.00 \\
0.51\end{array}$ & $\begin{array}{l}<0.02 \\
<0.02 \\
<0.02 \\
<0.01 \\
<0.01 \\
<0.02 \\
<0.02 \\
<0.02 \\
<0.02 \\
<0.01 \\
<0.01\end{array}$ & $\begin{array}{r}20.7 \\
14.6 \\
18.3 \\
66.1 \\
27 \\
<0.5 \\
1.5 \\
4.5 \\
<0.8 \\
<0.8 \\
<1.0\end{array}$ & $\begin{array}{l}0.10 \\
200 \\
0.10 \\
0.30 \\
0.30 \\
<0.02 \\
0.70 \\
0.40 \\
0.50 \\
0.00 \\
0.60\end{array}$ & $\begin{array}{l}<0.01 \\
<0.01 \\
<0.01 \\
<0.01 \\
<0.01 \\
<0.01 \\
<0.01 \\
<0.01 \\
<0.01 \\
0.01\end{array}$ & $\begin{array}{l}0 . .34 \\
0.02 \\
0.08 \\
<0.01 \\
0.04 \\
0.12 \\
0.03 \\
0.26 \\
0.11 \\
0.07\end{array}$ & $\begin{array}{l}0.30 \\
0.27 \\
0.23 \\
0.30 \\
0.50 \\
0.32 \\
0.65 \\
0.17 \\
0.91 \\
0.29 \\
0.66\end{array}$ & $\begin{array}{l}>0.20 \\
<0.20 \\
<0.20 \\
<0.20 \\
<0.20 \\
<0.20 \\
0.17 \\
0.54 \\
<0.02 \\
0.09\end{array}$ & $\begin{array}{l}0.81 \\
1.36 \\
0.13 \\
1.08 \\
0.19 \\
1.16 \\
1.08 \\
0.10 \\
1.85 \\
1.39 \\
0.54\end{array}$ & $\begin{array}{l}>0.01 \\
<0.01 \\
<0.01 \\
<0.01 \\
<0.01 \\
0.01 \\
0.01 \\
<0.01 \\
<0.01 \\
<0.01\end{array}$ & $\begin{array}{r}21.8 \\
20.9 \\
9.6 \\
6.8 \\
3.5 \\
6.9 \\
7.6 \\
7.6 \\
6.1 \\
4.8\end{array}$ & $\begin{array}{l}0.182 \\
0.048 \\
0.073 \\
0.139 \\
0.070 \\
0.136 \\
0.115 \\
0.153 \\
0.134 \\
0.100\end{array}$ & $\begin{array}{l}0.302 \\
0.390 \\
0.141 \\
0.073 \\
0.042 \\
0.072 \\
0.085 \\
0.084 \\
0.066 \\
0.054\end{array}$ & $\begin{array}{l}0.07 \\
-0.4927 \\
-1.4963 \\
-0.6377 \\
0.6231 \\
0.4954 \\
0.6122 \\
0.3009 \\
0.5819 \\
0.6795 \\
0.5877\end{array}$ \\
\hline $\begin{array}{l}\text { WC-16 } \\
\text { WC-21 } \\
\text { WC-22 } \\
\text { WC-35 } \\
\text { WC-48 } \\
\text { WC-68 } \\
\text { WC-89 } \\
\text { WC-105 } \\
\text { WC-118 } \\
\text { WC-130a } \\
\text { WC-142 }\end{array}$ & $\begin{array}{l}\text { VC-2B, Sul. Spgs. } \\
\text { VC-2B, Sul. Spgs. } \\
\text { VC-2B, Sul. Spgs. } \\
\text { VC-2B, Sul. Spgs. } \\
\text { VC-2B, Sul. Spgs. } \\
\text { VC-2B, Sul. Spgs. } \\
\text { VC-2B, Sul. Spgs. } \\
\text { VC-2B, Sul. Spgs. } \\
\text { VC-2B, Sul. Spgs. } \\
\text { vC-2B, Sul. Spgs. } \\
\text { VC-2B, Sul. Spgs. }\end{array}$ & $\begin{array}{l}090690 \\
1024490 \\
112690 \\
042391 \\
07 / 2991 \\
120591 \\
050191 \\
120292 \\
04292 \\
042193 \\
080393 \\
11 / 1693\end{array}$ & $\begin{array}{l}<0.02 \\
0.15 \\
<0.02 \\
<0.02 \\
0.31 \\
<0.05 \\
<0.05 \\
0.03 \\
<0.01 \\
0.04 \\
<0.02\end{array}$ & $\begin{array}{l}<0.05 \\
<0.05 \\
<0.02 \\
<0.01 \\
0.13 \\
<0.01 \\
<0.02 \\
<0.02 \\
<0.02 \\
<0.01 \\
<0.01\end{array}$ & $\begin{array}{l}\ldots . . \\
0.60 \\
0.20 \\
0.30 \\
2.30 \\
0.60 \\
2.00 \\
0.64 \\
0.53 \\
1.31 \\
0.43\end{array}$ & $\begin{array}{r}0.83 \\
0.59 \\
1.14 \\
0.00 \\
44.10 \\
0.29 \\
0.00 \\
0.00 \\
0.00 \\
0.16 \\
0.12\end{array}$ & $\begin{array}{l}<0.02 \\
<0.02 \\
<0.02 \\
0.03 \\
0.02 \\
<0.01 \\
<0.02 \\
<0.02 \\
<0.02 \\
<0.01 \\
<0.01\end{array}$ & $\begin{array}{r}18.3 \\
15.9 \\
14.6 \\
19.5 \\
6.1 \\
6.2 \\
<0.5 \\
4.0 \\
<0.8 \\
26 \\
<1.0\end{array}$ & $\begin{array}{l}200 \\
1.40 \\
0.60 \\
0.09 \\
6.80 \\
0.70 \\
0.50 \\
0.10 \\
0.20 \\
1.40 \\
0.80\end{array}$ & $\begin{array}{l}<-. . \\
<0.01 \\
0.01 \\
<0.01 \\
0.32 \\
<0.01 \\
<0.01 \\
<0.01 \\
0.03 \\
<0.01 \\
<0.01\end{array}$ & $\begin{array}{l}\ldots . . \\
0.11 \\
0.04 \\
<0.02 \\
0.17 \\
0.02 \\
0.03 \\
0.08 \\
0.08 \\
0.21 \\
0.05\end{array}$ & $\begin{array}{r}0.60 \\
0.67 \\
1.26 \\
0.23 \\
25.50 \\
0.30 \\
0.31 \\
0.07 \\
0.32 \\
0.71 \\
0.46\end{array}$ & $\begin{array}{r}\ldots . . \\
<0.20 \\
<0.20 \\
<0.20 \\
1.70 \\
<0.20 \\
<0.20 \\
0.30 \\
1.07 \\
<0.02 \\
0.15\end{array}$ & $\begin{array}{l}1.49 \\
1.70 \\
0.86 \\
0.63 \\
2.51 \\
0.63 \\
1.06 \\
0.11 \\
1.02 \\
2.82 \\
0.80\end{array}$ & $\begin{array}{l}\ldots .01 \\
0.01 \\
<0.01 \\
<0.01 \\
<0.01 \\
<0.01 \\
<0.01 \\
0.02 \\
0.07 \\
<0.01 \\
<0.01\end{array}$ & $\begin{array}{c}2 . .1 \\
20.1 \\
22.0 \\
108.0 \\
11.6 \\
55.2 \\
5.7 \\
4.2 \\
14.0 \\
4.9\end{array}$ & $\begin{array}{l}0.185 \\
0.108 \\
0.040 \\
1.609 \\
0.148 \\
0.160 \\
0.054 \\
0.067 \\
0.248 \\
0.123\end{array}$ & $\begin{array}{l}\ldots . . \\
0.466 \\
0.311 \\
0.352 \\
1.751 \\
0.157 \\
0.036 \\
0.074 \\
0.038 \\
0.175 \\
0.042\end{array}$ & $\begin{array}{c}-0.8649 \\
-0.9692 \\
-1.5932 \\
-0.0849 \\
-0.0587 \\
1.2666 \\
-0.3159 \\
0.5406 \\
0.3434 \\
0.9767\end{array}$ \\
\hline $\begin{array}{l}\text { WC-17 } \\
\text { WC-26 } \\
\text { WC-38 } \\
\text { WC-53 } \\
\text { WC-73 } \\
\text { WC-82 } \\
\text { WC-95 } \\
\text { WC-111 } \\
\text { WC-121 } \\
\text { WC-150 }\end{array}$ & $\begin{array}{l}\text { Tech Area } 49 \\
\text { Tech Area } 49 \\
\text { Tech Area } 49 \\
\text { Tech Area } 49 \\
\text { Tech Area } 49 \\
\text { Tech Area } 49 \\
\text { Tech Area } 49 \\
\text { Tech Area } 49 \\
\text { Tech Area } 49 \\
\text { Tech Area } 49\end{array}$ & 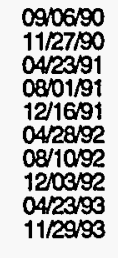 & $\begin{array}{l}<0.02 \\
<0.02 \\
<0.02 \\
<0.05 \\
<0.05 \\
<0.05 \\
<0.05 \\
<0.01 \\
<0.01 \\
<0.02\end{array}$ & $\begin{array}{l}<0.05 \\
<0.02 \\
<0.01 \\
<0.01 \\
<0.01 \\
<0.02 \\
<0.02 \\
<0.02 \\
<0.02 \\
<0.01\end{array}$ & $\begin{array}{l}\ldots . \\
0.60 \\
0.60 \\
0.80 \\
0.90 \\
0.90 \\
1.50 \\
1.07 \\
0.39 \\
0.69\end{array}$ & $\begin{array}{l}0.06 \\
0.00 \\
0.00 \\
0.00 \\
0.55 \\
0.29 \\
0.11 \\
0.13 \\
0.00 \\
0.06\end{array}$ & $\begin{array}{l}<0.02 \\
<0.02 \\
<0.02 \\
<0.01 \\
<0.01 \\
<0.02 \\
<0.02 \\
<0.02 \\
<0.02 \\
<0.01\end{array}$ & $\begin{array}{r}22.0 \\
13.4 \\
19.5 \\
11.0 \\
4.3 \\
3.4 \\
19.9 \\
21.5 \\
<0.8 \\
<1.0\end{array}$ & $\begin{array}{l}1.00 \\
0.63 \\
0.20 \\
0.40 \\
0.80 \\
0.60 \\
1.00 \\
3.20 \\
0.10 \\
1.80\end{array}$ & $\begin{array}{l}\quad \ldots \\
<0.01 \\
<0.01 \\
<0.01 \\
<0.01 \\
<0.01 \\
<0.01 \\
<0.01 \\
0.01 \\
<0.01\end{array}$ & $\begin{array}{l}\ldots . .14 \\
0.14 \\
0.10 \\
0.06 \\
0.07 \\
0.19 \\
0.16 \\
0.01 \\
0.19\end{array}$ & $\begin{array}{l}0.20 \\
0.17 \\
0.26 \\
0.30 \\
0.90 \\
0.86 \\
0.55 \\
0.52 \\
0.28 \\
0.24\end{array}$ & $\begin{array}{l}\ldots 0 . \\
<0.20 \\
<0.20 \\
<0.20 \\
<0.20 \\
<0.20 \\
<0.20 \\
0.24 \\
0.04 \\
0.21\end{array}$ & $\begin{array}{l}1.72 \\
1.19 \\
1.39 \\
1.64 \\
0.96 \\
2.00 \\
2.39 \\
1.78 \\
1.62 \\
1.87\end{array}$ & $\begin{array}{c}<0.01 \\
<0.01 \\
<0.01 \\
<0.01 \\
<0.01 \\
0.01 \\
<0.01 \\
<0.01 \\
0.01\end{array}$ & $\begin{array}{r}18.5 \\
24.9 \\
17.2 \\
11.0 \\
11.2 \\
35.1 \\
42.5 \\
4.6 \\
32.2\end{array}$ & $\begin{array}{l}0.118 \\
0.099 \\
0.147 \\
0.160 \\
0.176 \\
0.452 \\
0.730 \\
0.085 \\
0.807\end{array}$ & $\begin{array}{l}\ldots . . \\
0.277 \\
0.396 \\
0.257 \\
0.143 \\
0.146 \\
0.497 \\
0.518 \\
0.063 \\
0.393\end{array}$ & $\begin{array}{r}-0.8076 \\
-1.1989 \\
-0.5452 \\
0.1145 \\
0.1871 \\
-0.0935 \\
0.3402 \\
0.2950 \\
0.6902\end{array}$ \\
\hline $\begin{array}{l}\text { WC-18 } \\
\text { WC-27 } \\
\text { WC-40 } \\
\text { WC-59. } \\
\text { WC-75 } \\
\text { WC-88 } \\
\text { WC-100 } \\
\text { WC-109 } \\
\text { WC-139 } \\
\text { WC-151 }\end{array}$ & $\begin{array}{l}\text { Tech Area } 51 \\
\text { Tech Area } 51 \\
\text { Tech Area 51 } \\
\text { Tech Area 51 } \\
\text { Tech Area 51 } \\
\text { Tech Area } 51 \\
\text { Tech Area 51 } \\
\text { Tech Area 51 } \\
\text { Tech Area 51 } \\
\text { Tech Area } 51\end{array}$ & $\begin{array}{l}09106900 \\
112790 \\
0424191 \\
0810591 \\
1211791 \\
042992 \\
0814192 \\
120392 \\
0811399 \\
11 / 2999\end{array}$ & $\begin{array}{l}<0.02 \\
<0.02 \\
<0.02 \\
<0.05 \\
<0.05 \\
<0.05 \\
<0.05 \\
<0.01 \\
<0.01 \\
<0.02\end{array}$ & $\begin{array}{l}<0.05 \\
<0.02 \\
<0.01 \\
<0.01 \\
<0.01 \\
<0.02 \\
<0.02 \\
<0.02 \\
<0.01 \\
<0.01\end{array}$ & $\begin{array}{l}\ldots . \\
0.60 \\
0.40 \\
0.80 \\
0.50 \\
0.60 \\
1.30 \\
1.25 \\
1.54 \\
0.41\end{array}$ & $\begin{array}{l}0.00 \\
0.00 \\
0.00 \\
0.00 \\
0.47 \\
0.10 \\
0.00 \\
0.00 \\
0.00 \\
0.00\end{array}$ & $\begin{array}{l}<0.02 \\
<0.02 \\
<0.02 \\
<0.01 \\
<0.01 \\
<0.02 \\
<0.02 \\
<0.02 \\
<0.01 \\
<0.01\end{array}$ & $\begin{array}{r}18.3 \\
14.6 \\
19.5 \\
7.3 \\
0.6 \\
00.5 \\
<0.5 \\
1.8 \\
<0.5 \\
2.8 \\
<1.0\end{array}$ & $\begin{array}{l}0.20 \\
0.12 \\
0.10 \\
0.50 \\
0.40 \\
0.50 \\
0.60 \\
0.30 \\
0.60 \\
0.20\end{array}$ & $\begin{array}{l}\ldots 0 . \\
<0.01 \\
<0.01 \\
<0.01 \\
<0.01 \\
<0.01 \\
<0.01 \\
<0.01 \\
<0.01 \\
0.16\end{array}$ & $\begin{array}{l}\ldots . \\
0.12 \\
0.06 \\
0.08 \\
<0.01 \\
0.04 \\
0.12 \\
0.07 \\
0.13 \\
0.05\end{array}$ & $\begin{array}{l}0.20 \\
0.14 \\
0.53 \\
0.30 \\
1.00 \\
0.67 \\
0.40 \\
0.20 \\
0.37 \\
0.18\end{array}$ & $\begin{array}{l}\ldots . . \\
<0.20 \\
<0.20 \\
<0.20 \\
<0.20 \\
<0.20 \\
<0.20 \\
0.28 \\
<0.02 \\
<0.10\end{array}$ & $\begin{array}{l}0.96 \\
0.85 \\
0.84 \\
1.05 \\
0.76 \\
1.21 \\
1.38 \\
1.73 \\
1.55 \\
0.80\end{array}$ & $\begin{array}{l}<0.01 \\
<0.01 \\
<0.01 \\
<0.01 \\
<0.01 \\
<0.01 \\
<0.01 \\
<0.01 \\
<0.01\end{array}$ & $\begin{array}{r}17.7 \\
23.7 \\
11.2 \\
5.1 \\
5.2 \\
7.7 \\
5.7 \\
12.4 \\
3.5\end{array}$ & $\begin{array}{l}0.071 \\
0.093 \\
0.088 \\
0.091 \\
0.100 \\
0.138 \\
0.107 \\
0.210 \\
0.098\end{array}$ & $\begin{array}{l}0.274 \\
0.369 \\
0.159 \\
0.069 \\
0.059 \\
0.006 \\
0.063 \\
0.149 \\
0.039\end{array}$ & $\begin{array}{r}-1.1739 \\
-1.1916 \\
-0.5741 \\
0.3621 \\
0.5115 \\
0.4609 \\
0.5257 \\
0.3395 \\
0.8614\end{array}$ \\
\hline $\begin{array}{l}\text { WC-60 } \\
\text { WC-74 } \\
\text { WC-91 } \\
\text { WC-104 } \\
\text { WC-117 } \\
\text { WC-120 } \\
\text { WC-141 } \\
\text { WC-154 } \\
\end{array}$ & $\begin{array}{l}\text { K. Manley's House } \\
\text { K. Manleys House } \\
\text { K. Manleys House } \\
\text { K. Manley's House } \\
\text { K. Manley's House } \\
\text { K. Manley's House } \\
\text { K. Manley's House } \\
\text { K. Manley's House } \\
\end{array}$ & $\begin{array}{l}08059191 \\
121591 \\
05127192 \\
0820992 \\
121892 \\
0412393 \\
08 / 1399 \\
1113096 \\
\end{array}$ & $\begin{array}{l}<0.05 \\
<0.05 \\
<0.05 \\
<0.05 \\
<0.01 \\
<0.01 \\
<0.01 \\
<0.02 \\
\end{array}$ & $\begin{array}{l}<0.01 \\
<0.01 \\
<0.02 \\
<0.02 \\
<0.02 \\
<0.02 \\
<0.01 \\
<0.01\end{array}$ & $\begin{array}{l}0.50 \\
0.80 \\
0.60 \\
1.00 \\
0.41 \\
0.44 \\
1.27 \\
0.50 \\
\end{array}$ & $\begin{array}{l}0.00 \\
0.53 \\
1.71 \\
0.11 \\
0.00 \\
0.00 \\
0.00 \\
0.00 \\
\end{array}$ & $\begin{array}{l}<0.01 \\
<0.01 \\
<0.02 \\
<0.02 \\
<0.02 \\
<0.02 \\
<0.01 \\
<0.01 \\
\end{array}$ & $\begin{array}{r}6.1 \\
<0.5 \\
<0.5 \\
23 \\
20.5 \\
<0.8 \\
<0.8 \\
25 \\
<1.0 \\
\end{array}$ & $\begin{array}{l}0.50 \\
0.60 \\
0.90 \\
1.20 \\
0.10 \\
0.10 \\
1.40 \\
0.40 \\
\end{array}$ & $\begin{array}{l}<0.01 \\
<0.01 \\
<0.01 \\
<0.01 \\
<0.01 \\
0.02 \\
<0.01 \\
<0.01 \\
\end{array}$ & $\begin{array}{l}0.04 \\
<0.01 \\
0.07 \\
0.16 \\
0.01 \\
0.03 \\
0.21 \\
0.04 \\
\end{array}$ & $\begin{array}{l}0.30 \\
1.00 \\
1.14 \\
0.62 \\
0.11 \\
0.26 \\
0.36 \\
0.09 \\
\end{array}$ & $\begin{array}{r}<0.20 \\
<0.20 \\
<0.20 \\
<0.20 \\
0.06 \\
0.04 \\
<0.02 \\
<0.10 \\
\end{array}$ & $\begin{array}{l}0.67 \\
0.79 \\
0.97 \\
1.39 \\
0.06 \\
0.85 \\
1.02 \\
0.73 \\
\end{array}$ & $\begin{array}{l}<0.01 \\
<0.01 \\
<0.01 \\
0.04 \\
<0.01 \\
0.01 \\
<0.01 \\
<0.01 \\
\end{array}$ & $\begin{array}{r}9.1 \\
5.1 \\
7.3 \\
9.1 \\
1.1 \\
2.9 \\
10.7 \\
3.0 \\
\end{array}$ & $\begin{array}{l}0.061 \\
0.108 \\
0.129 \\
0.155 \\
0.037 \\
0.056 \\
0.194 \\
0.059 \\
\end{array}$ & $\begin{array}{l}0.133 \\
0.056 \\
0.096 \\
0.106 \\
0.006 \\
0.035 \\
0.123 \\
0.034 \\
\end{array}$ & $\begin{array}{l}-0.7384 \\
0.6409 \\
0.2809 \\
0.3772 \\
1.4612 \\
0.4462 \\
0.4461 \\
0.5469 \\
\end{array}$ \\
\hline
\end{tabular}

(a) Analyses by $D$. Counce and P. E. Trujtllo.
(b) Chioride values corrected for chloride leached from bags (determined from blank analyses). 


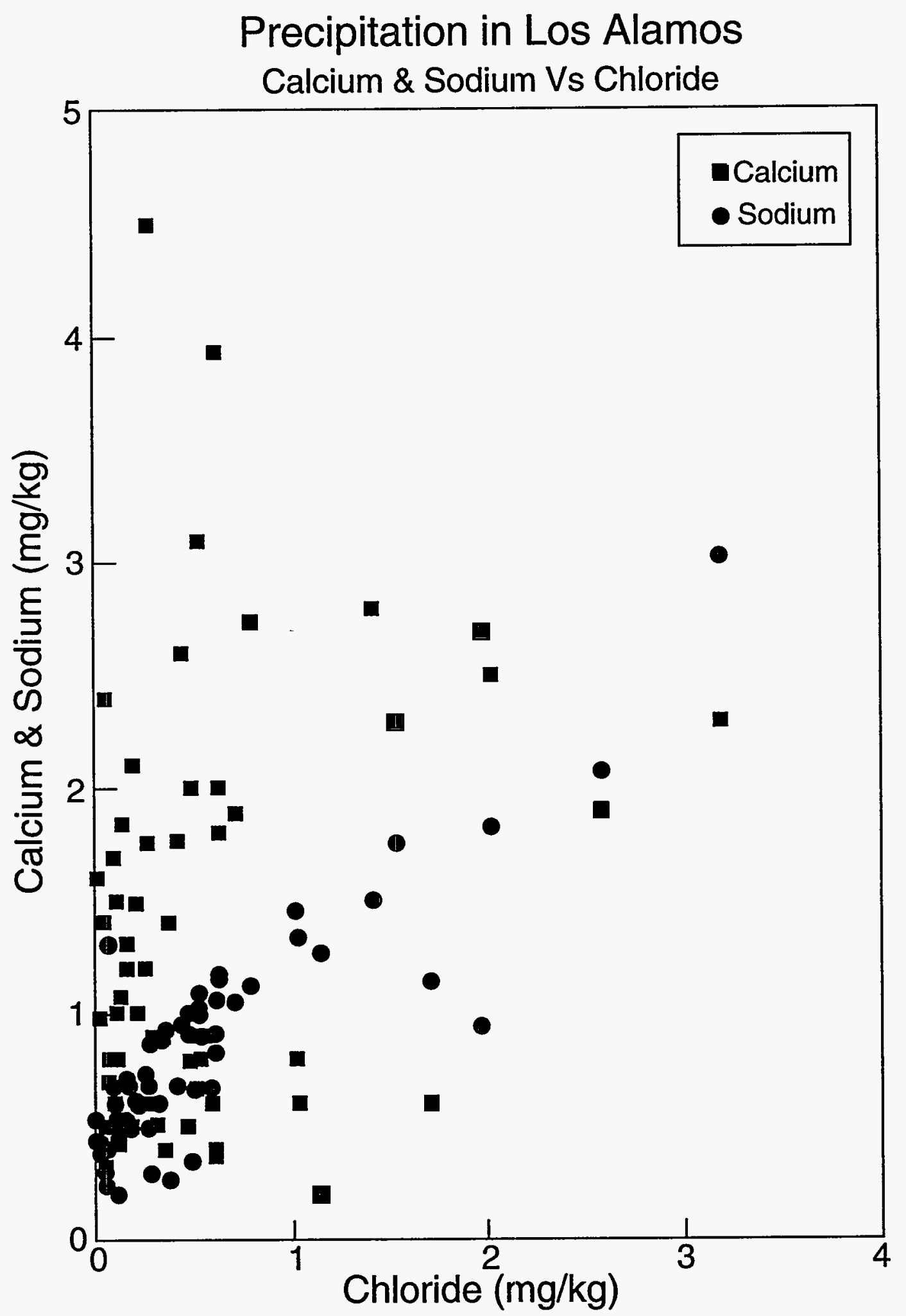

Figure 4: Plots of sodium and calcium versus chloride for precipitation in the Los Alamos region. 
TABLE 3. Trace Element Chemistry for Precipitation in the Los Alamos Region, New Mexico $(a, b)$

\begin{tabular}{|c|c|c|c|c|c|c|c|c|c|c|c|c|c|c|c|}
\hline Sample\# & Description & Date & $\begin{array}{c}\mathrm{Ag} \\
\mathrm{mg} / \mathrm{kg}\end{array}$ & $\begin{array}{r}\mathrm{Al} \\
\mathrm{mg} / \mathrm{kg} \\
\end{array}$ & $\begin{array}{c}\mathrm{As} \\
\mathrm{mg} / \mathrm{kg}\end{array}$ & $\begin{array}{c}\mathrm{Ba} \\
\mathrm{mg} / \mathrm{kg}\end{array}$ & $\begin{array}{c}\mathrm{Cd} \\
\mathrm{mg} / \mathrm{kg}\end{array}$ & $\begin{array}{c}\mathrm{Co} \\
\mathrm{mg} / \mathrm{kg}\end{array}$ & $\begin{array}{c}\mathrm{Cr} \\
\mathrm{mg} / \mathrm{kg}\end{array}$ & $\begin{array}{c}\mathrm{Cs} \\
\mathrm{mg} / \mathrm{kg}\end{array}$ & $\begin{array}{c}\mathrm{Cu} \\
\mathrm{mg} / \mathrm{kg}\end{array}$ & $\begin{array}{c}\mathrm{Fe} \\
\mathrm{mg} / \mathrm{kg}\end{array}$ & $\begin{array}{c}\mathrm{Hg} \\
\mathrm{mg} / \mathrm{kg}\end{array}$ & $\begin{array}{c}1 \\
\mathrm{mg} / \mathrm{kg}\end{array}$ & $\begin{array}{c}\mathrm{Mn} \\
\mathrm{mg} / \mathrm{kg}\end{array}$ \\
\hline & Drinking Water Criteria & $\cdots$ & 0.1 & $\cdots$ & 0.05 & 1 & 0.01 & $\cdots$ & 0.1 & -- & 1 & 0.3 & 0.002 & $\cdots$ & 0.05 \\
\hline $\begin{array}{l}\text { WC-1 } \\
\text { WC-9 } \\
\text { WC-28 } \\
\text { WC-46 } \\
\text { WC-51 } \\
\text { WC-67 } \\
\text { WC-80 } \\
\text { WC-97 } \\
\text { WC-113 } \\
\text { WC-146 }\end{array}$ & $\begin{array}{l}\text { Santa Fe Airport } \\
\text { Santa Fe Airport } \\
\text { Santa Fe Airport } \\
\text { Santa Fe Airport } \\
\text { Santa Fe Airport } \\
\text { Santa Fe Airport } \\
\text { Santa Fe Airport } \\
\text { Santa Fe Airport } \\
\text { Santa Fe Airport } \\
\text { Santa } F e \text { Airport }\end{array}$ & $\begin{array}{l}05 / 23 / 90 \\
08 / 09 / 90 \\
11 / 28 / 90 \\
04 / 25 / 91 \\
07 / 29 / 91 \\
12 / 04 / 91 \\
04 / 20 / 92 \\
08 / 14 / 92 \\
12 / 04 / 92 \\
11 / 18 / 93\end{array}$ & $\begin{array}{c}\ldots .- \\
\ldots . \\
\ldots . \\
\cdots \\
\cdots \\
<0.001 \\
<0.001 \\
<0.001 \\
<0.0005\end{array}$ & $\begin{array}{l}\quad \cdots \\
<0.1 \\
<0.1 \\
<0.1 \\
<0.1 \\
<0.1 \\
<0.1 \\
<0.1 \\
<0.05\end{array}$ & $\begin{array}{c}\quad \ldots \\
<0.05 \\
<0.05 \\
<0.05 \\
<0.05 \\
<0.05 \\
<0.05 \\
<0.05 \\
0.0006\end{array}$ & $\begin{array}{l}\quad . . \\
<0.01 \\
<0.01 \\
<0.02 \\
<0.01 \\
<0.01 \\
<0.01 \\
<0.01 \\
<0.01\end{array}$ & $\begin{array}{c}\cdots \\
\cdots \\
\cdots \\
\cdots . \\
\cdots \\
\cdots \\
<0.001 \\
<0.001 \\
<0.001 \\
<0.0005\end{array}$ & $\begin{array}{l}\ldots .- \\
-. . \\
\cdots \\
\cdots \\
\cdots \\
\cdots \\
<0.002 \\
<0.002 \\
<0.002 \\
<0.002\end{array}$ & $\begin{array}{c}\ldots . \\
\ldots . \\
\ldots . \\
\ldots- \\
\ldots . \\
\ldots 0.002 \\
<0.002 \\
<0.002 \\
<0.002\end{array}$ & $\begin{array}{c}\ldots \\
\ldots \\
\ldots . \\
\ldots \\
-. \\
\cdots \\
<0.002 \\
<0.002 \\
<0.002 \\
<0.002\end{array}$ & $\begin{array}{c}\cdots \\
\cdots \\
\cdots- \\
\cdots \\
\cdots- \\
-. \\
0.010 \\
0.005 \\
<0.002 \\
0.010\end{array}$ & $\begin{array}{r}\quad \ldots \\
<0.01 \\
<0.01 \\
<0.02 \\
<0.01 \\
0.08 \\
0.02 \\
<0.01 \\
0.02\end{array}$ & $\begin{array}{c}\cdots \\
<0.1 \\
<0.1 \\
\ldots . \\
\cdots \\
\cdots \\
\cdots \\
\cdots \\
<0.0005\end{array}$ & $\begin{array}{c}\cdots \\
\cdots \\
\cdots \\
\cdots \\
\cdots \\
\cdots \\
- \\
<0.02 \\
<0.01 \\
<0.01\end{array}$ & $\begin{array}{l}\quad-. \\
-.- \\
0.01 \\
<0.01 \\
<0.01 \\
<0.01 \\
<0.01 \\
<0.01 \\
<0.01 \\
<0.01\end{array}$ \\
\hline $\begin{array}{l}\text { WC-2 } \\
\text { WC-8 } \\
\text { WC-25 } \\
\text { WC-37 } \\
\text { WC-54 } \\
\text { WC-70 } \\
\text { WC-83 } \\
\text { WC-102 } \\
\text { WC-110 } \\
\text { WC-125 } \\
\text { WC-138 } \\
\text { WC-149 }\end{array}$ & $\begin{array}{l}\text { Tech Area } 33 \\
\text { Tech Area } 33 \\
\text { Tech Area } 33 \\
\text { Tech Area } 33 \\
\text { Tech Area } 33 \\
\text { Tech Area } 33 \\
\text { Tech Area } 33 \\
\text { Tech Area } 33 \\
\text { Tech Area } 33 \\
\text { Tech Area } 33 \\
\text { Tech Area } 33 \\
\text { Tech Area } 33\end{array}$ & $\begin{array}{l}05 / 23 / 90 \\
08 / 09 / 90 \\
11 / 27 / 90 \\
04 / 23 / 91 \\
08 / 01 / 91 \\
12 / 09 / 91 \\
04 / 20 / 92 \\
08 / 20 / 92 \\
12 / 03 / 92 \\
04 / 28 / 93 \\
08 / 09 / 93 \\
12 / 03 / 93\end{array}$ & $\begin{array}{c}\ldots . . \\
\cdots . . \\
\cdots \\
\cdots . \\
\cdots . . \\
<0.001 \\
<0.001 \\
<0.001 \\
<0.001 \\
<0.0005 \\
<0.0005\end{array}$ & $\begin{array}{l}\quad \ldots \\
\quad-. \\
<0.1 \\
<0.1 \\
<0.1 \\
<0.1 \\
<0.1 \\
<0.1 \\
<0.1 \\
<0.1 \\
<0.1 \\
0.06\end{array}$ & $\begin{array}{c}\ldots . \\
\ldots . \\
<0.05 \\
<0.05 \\
<0.05 \\
<0.05 \\
<0.05 \\
<0.05 \\
<0.05 \\
<0.05 \\
<0.05 \\
0.006\end{array}$ & $\begin{array}{l}\quad \cdots \\
<0.01 \\
<0.01 \\
<0.02 \\
<0.01 \\
<0.01 \\
<0.01 \\
<0.01 \\
<0.01 \\
<0.01 \\
<0.01\end{array}$ & $\begin{array}{c}\ldots . \\
\ldots . \\
\ldots . \\
\ldots . \\
\ldots . \\
0.001 \\
<0.001 \\
<0.001 \\
0.003 \\
0.0009 \\
<0.0005\end{array}$ & $\begin{array}{c}\cdots \\
\cdots \\
\cdots \\
\cdots \\
\cdots \\
\cdots \\
<0.002 \\
<0.002 \\
<0.002 \\
<0.002 \\
<0.002 \\
<0.002\end{array}$ & $\begin{array}{c}\ldots . \\
\ldots . \\
\ldots . \\
\ldots . \\
\ldots . . \\
<0.002 \\
<0.002 \\
<0.002 \\
<0.002 \\
<0.002 \\
<0.002\end{array}$ & $\begin{array}{c}\cdots \\
\cdots \\
\cdots \\
\cdots \\
\cdots \\
\cdots \\
<0.002 \\
0.003 \\
<0.002 \\
<0.002 \\
<0.002 \\
<0.002\end{array}$ & $\begin{array}{c}\cdots \\
\cdots \\
\cdots- \\
\cdots- \\
\cdots \\
\cdots . . \\
0.003 \\
0.005 \\
0.002 \\
0.010 \\
0.005 \\
0.005\end{array}$ & $\begin{array}{r}\cdots \\
<0.01 \\
<0.01 \\
<0.02 \\
0.01 \\
<0.01 \\
<0.01 \\
<0.01 \\
<0.01 \\
0.02 \\
0.01\end{array}$ & $\begin{array}{c}\ldots . . \\
<0.1 \\
<0.1 \\
\ldots . \\
\cdots . \\
\cdots . \\
\cdots \\
\cdots \\
\cdots \\
<0.0005\end{array}$ & $\begin{array}{c}\cdots \\
\cdots \\
\cdots \\
\cdots \\
\cdots \\
\cdots \\
<0.0 \\
<0.01 \\
<0.01 \\
<0.01 \\
<0.01\end{array}$ & $\begin{array}{l}\quad \cdots \\
<0.01 \\
<0.01 \\
<0.01 \\
<0.01 \\
<0.01 \\
<0.01 \\
<0.01 \\
<0.01 \\
<0.01 \\
<0.01\end{array}$ \\
\hline $\begin{array}{l}\text { WC-3 } \\
\text { WC-19 } \\
\text { WC-33 } \\
\text { WC-42 } \\
\text { WC-57 } \\
\text { WC-77 } \\
\text { WC-79 } \\
\text { WC-99 } \\
\text { WC-107 } \\
\text { WC-127 } \\
\text { WC-140 } \\
\text { WC-152 }\end{array}$ & $\begin{array}{l}\text { Philomena's } \\
\text { Philomena's } \\
\text { Philomena's } \\
\text { Philomena's } \\
\text { Philomena's } \\
\text { Philomena's } \\
\text { Philomena's } \\
\text { Philomena's } \\
\text { Philomena's } \\
\text { Philomena's } \\
\text { Philomena's } \\
\text { Philomena's }\end{array}$ & $\begin{array}{l}05 / 23 / 90 \\
09 / 07 / 90 \\
12 / 04 / 90 \\
04 / 24 / 91 \\
08 / 05 / 91 \\
12 / 19 / 91 \\
03 / 31 / 92 \\
08 / 14 / 92 \\
12 / 03 / 92 \\
04 / 29 / 93 \\
08 / 13 / 93 \\
11 / 29 / 93\end{array}$ & 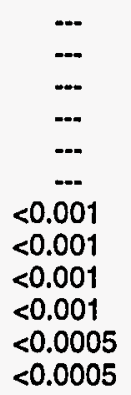 & $\begin{array}{l}\quad \ldots \\
<0.1 \\
<0.1 \\
<0.1 \\
<0.1 \\
<0.1 \\
<0.1 \\
<0.1 \\
<0.1 \\
<0.1 \\
<0.05\end{array}$ & $\begin{array}{c}\quad \ldots \\
<0.05 \\
<0.05 \\
<0.05 \\
<0.05 \\
<0.05 \\
<0.05 \\
<0.05 \\
<0.05 \\
0.12 \\
0.006\end{array}$ & $\begin{array}{c}\quad-- \\
<0.01 \\
<0.01 \\
<0.02 \\
<0.01 \\
<0.01 \\
0.02 \\
<0.01 \\
<0.01 \\
<0.01 \\
<0.01\end{array}$ & $\begin{array}{c}\ldots . \\
\ldots . \\
\ldots . \\
\ldots . \\
\ldots . . \\
<0.001 \\
<0.001 \\
<0.001 \\
<0.001 \\
0.0002 \\
0.0006\end{array}$ & $\begin{array}{c}\ldots . \\
\ldots \\
\ldots . . \\
\ldots . . \\
<0.002 \\
<0.002 \\
<0.002 \\
<0.002 \\
<0.002 \\
<0.002\end{array}$ & $\begin{array}{c}\ldots \\
\cdots . . \\
\cdots \\
\cdots \\
\cdots \\
0 . . \\
0003 \\
<0.002 \\
<0.002 \\
<0.002 \\
<0.002 \\
<0.002\end{array}$ & $\begin{array}{c}\cdots \\
\cdots \\
\cdots \\
\cdots \\
\cdots \\
\cdots \\
<0.002 \\
0.004 \\
<0.002 \\
<0.002 \\
<0.002 \\
<0.002\end{array}$ & $\begin{array}{c}\cdots \\
\cdots . \\
\cdots . \\
\ldots . \\
\cdots . \\
0.007 \\
0.005 \\
0.004 \\
0.003 \\
0.009 \\
0.008\end{array}$ & $\begin{array}{r}\cdots \\
<0.01 \\
<0.01 \\
0.02 \\
0.01 \\
<0.01 \\
<0.01 \\
<0.01 \\
<0.01 \\
0.05 \\
0.01\end{array}$ & $\begin{array}{c}\ldots . . \\
<0.1 \\
<0.1 \\
\ldots . . \\
\cdots . \\
\ldots . \\
\ldots . \\
\cdots \\
\cdots \\
<0.0005\end{array}$ & $\begin{array}{l}\ldots . . \\
\ldots \\
\ldots \\
\ldots \\
\ldots \\
\ldots \\
<0.02 \\
<0.01 \\
<0.01 \\
<0.01 \\
<0.01\end{array}$ & $\begin{array}{r}\quad \cdots \\
<0.01 \\
<0.01 \\
<0.01 \\
0.01 \\
<0.01 \\
<0.01 \\
<0.01 \\
<0.01 \\
0.02 \\
<0.01\end{array}$ \\
\hline $\begin{array}{l}\text { WC-4 } \\
\text { WC-20 } \\
\text { WC-34 } \\
\text { WC-45 }\end{array}$ & $\begin{array}{l}\text { Pajarito Mountain } \\
\text { Pajarito Mountain } \\
\text { Pajarito Mountain } \\
\text { Pajarito Mountain }\end{array}$ & $\begin{array}{l}05 / 23 / 90 \\
09 / 07 / 90 \\
12 / 07 / 90 \\
04 / 25 / 91\end{array}$ & $\begin{array}{l}\cdots \\
\cdots \\
\cdots\end{array}$ & $\begin{array}{r}\cdots \\
<0.1 \\
0.3\end{array}$ & $\begin{array}{r}\ldots . \\
<0.05 \\
<0.05\end{array}$ & $\begin{array}{r}\ldots .01 \\
0.0 .01\end{array}$ & $\begin{array}{l}\cdots \\
\cdots \\
\cdots\end{array}$ & $\begin{array}{l}\cdots \\
\cdots \\
\cdots\end{array}$ & $\begin{array}{l}\cdots \\
\cdots \\
\cdots\end{array}$ & $\begin{array}{l}\cdots \\
\cdots \\
\cdots\end{array}$ & $\begin{array}{l}\cdots \\
\cdots \\
\cdots\end{array}$ & $\begin{array}{r}\cdots \\
<0.01 \\
0.12\end{array}$ & $\begin{array}{l}\quad \cdots \\
<0.1 \\
<0.1\end{array}$ & $\cdots$ & $\begin{array}{r}\ldots .05 \\
0.0 . \\
<0.01\end{array}$ \\
\hline
\end{tabular}




\begin{tabular}{|c|c|c|c|c|c|c|c|c|c|c|c|c|c|c|c|c|}
\hline Sample \# & Description & Date & $\begin{array}{c}\mathrm{Mo} \\
\mathrm{mg} / \mathrm{kg}\end{array}$ & $\begin{array}{c}\mathrm{NH4} \\
\mathrm{mg} / \mathrm{kg}\end{array}$ & $\begin{array}{c}\mathrm{Ni} \\
\mathrm{mg} / \mathrm{kg}\end{array}$ & $\begin{array}{c}\mathrm{NO2} \\
\mathrm{mg} / \mathrm{kg}\end{array}$ & $\begin{array}{c}\mathrm{NO3} \\
\mathrm{mg} / \mathrm{kg}\end{array}$ & $\begin{array}{c}\mathrm{Pb} \\
\mathrm{mg} / \mathrm{kg}\end{array}$ & $\begin{array}{c}\mathrm{PO4} \\
\mathrm{mg} / \mathrm{kg}\end{array}$ & $\begin{array}{c}\mathrm{Rb} \\
\mathrm{mg} / \mathrm{kg}\end{array}$ & $\begin{array}{c}\mathrm{Sb} \\
\mathrm{mg} / \mathrm{kg}\end{array}$ & $\begin{array}{c}\mathrm{Se} \\
\mathrm{mg} / \mathrm{kg}\end{array}$ & $\begin{array}{c}\mathrm{Si} \\
\mathrm{mg} / \mathrm{kg}\end{array}$ & $\begin{array}{l}\mathrm{S} 2 \mathrm{O} 3 \\
\mathrm{mg} / \mathrm{kg}\end{array}$ & $\underset{\mathrm{mg} / \mathrm{kg}}{\mathrm{U}}$ & $\begin{array}{c}\mathrm{Zn} \\
\mathrm{mg} / \mathrm{kg}\end{array}$ \\
\hline & Drinking Water Criteria & $\ldots$ & $\cdots$ & $\cdots$ & $\cdots$ & $-\infty$ & 45 & 0.05 & - & $\ldots$ & $\cdots$ & 0.01 & - & - & $\cdots$ & 5 \\
\hline $\begin{array}{l}\text { WC-1 } \\
\text { WC-9 } \\
\text { WC-28 } \\
\text { WC-46 } \\
\text { WC-51 } \\
\text { WC-67 } \\
\text { WC-80 } \\
\text { WC-97 } \\
\text { WC-113 } \\
\text { WC-146 }\end{array}$ & 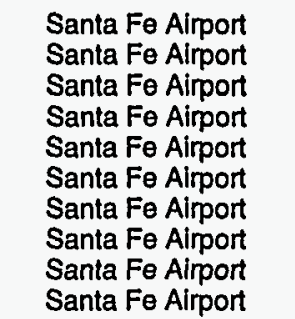 & $\begin{array}{l}05 / 23 / 90 \\
08 / 09 / 90 \\
11 / 28 / 90 \\
04 / 25 / 91 \\
07 / 29 / 91 \\
12 / 04 / 91 \\
04 / 20 / 92 \\
08 / 14 / 92 \\
12 / 04 / 92 \\
11 / 18 / 93\end{array}$ & $\begin{array}{c}-. . \\
-- \\
-- \\
\cdots \\
\cdots \\
<0.002 \\
<0.002 \\
<0.002 \\
<0.002\end{array}$ & $\begin{array}{r}1.08 \\
0.35 \\
1.40 \\
3.18 \\
0.27 \\
0.22 \\
7.56 \\
1.13 \\
22.50 \\
3.09\end{array}$ & $\begin{array}{c}\ldots \\
\ldots \\
- \\
- \\
\cdots \\
\cdots \\
0.023 \\
<0.002 \\
<0.002 \\
0.002\end{array}$ & \begin{tabular}{r}
\multicolumn{1}{c}{} \\
9.1 \\
0.26 \\
0.07 \\
0.20 \\
0.05 \\
0.02 \\
$<0.02$ \\
$<0.02$ \\
4.16
\end{tabular} & $\begin{array}{l}1.18 \\
2.99 \\
2.83 \\
2.18 \\
0.82 \\
1.93 \\
1.48 \\
7.01 \\
1.54 \\
2.05\end{array}$ & $\begin{array}{c}\cdots \\
\cdots \\
\cdots \\
\cdots \\
\cdots \\
<0.002 \\
<0.002 \\
0.002 \\
0.005\end{array}$ & $\begin{array}{c}0.17 \\
0.26 \\
<0.05 \\
1.63 \\
<0.02 \\
0.29 \\
<0.05 \\
0.58 \\
3.44 \\
0.24\end{array}$ & $\begin{array}{l}- \\
\overline{-} \\
- \\
\overline{-} \\
<0.002 \\
<0.002 \\
<0.002 \\
0.004\end{array}$ & 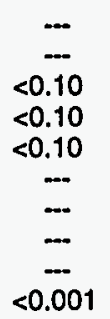 & $\begin{array}{c}\overline{-} \\
<0 . \overline{10} \\
<0.10 \\
\cdots \\
\cdots \\
\cdots \\
\overline{-} \\
- \\
<001\end{array}$ & $\begin{array}{l}\quad \ldots \\
<0.1 \\
<0.1 \\
<0.1 \\
<0.1 \\
<0.1 \\
0.1 \\
0.15 \\
0.08\end{array}$ & $\begin{array}{l}\overline{-} \\
\overline{-} \\
\cdots \\
\cdots \\
\cdots \\
<0.02 \\
<0.01 \\
<0.01\end{array}$ & $\begin{array}{l}- \\
0.1 \\
<0.1 \\
- \\
\overline{-} \\
\overline{-} \\
- \\
-\end{array}$ & $\begin{array}{l}-\overline{-} \\
1 . \overline{70} \\
0.03 \\
0.01 \\
0.02 \\
0.05 \\
0.05 \\
0.03 \\
0.23\end{array}$ \\
\hline $\begin{array}{l}\text { WC-2 } \\
\text { WC-8 } \\
\text { WC-25 } \\
\text { WC-37 } \\
\text { WC-54 } \\
\text { WC-70 } \\
\text { WC-83 } \\
\text { WC-102 } \\
\text { WC-110 } \\
\text { WC-125 } \\
\text { WC-138 } \\
\text { WC-149 }\end{array}$ & $\begin{array}{l}\text { Tech Area } 33 \\
\text { Tech Area } 33 \\
\text { Tech Area } 33 \\
\text { Tech Area } 33 \\
\text { Tech Area } 33 \\
\text { Tech Area } 33 \\
\text { Tech Area } 33 \\
\text { Tech Area } 33 \\
\text { Tech Area } 33 \\
\text { Tech Area } 33 \\
\text { Tech Area } 33 \\
\text { Tech Area } 33\end{array}$ & $\begin{array}{l}05 / 23 / 90 \\
08 / 09 / 90 \\
11 / 27 / 90 \\
04 / 23 / 91 \\
08 / 01 / 91 \\
12 / 09 / 91 \\
04 / 20 / 92 \\
08 / 20 / 92 \\
12 / 03 / 92 \\
04 / 28 / 93 \\
08 / 09 / 93 \\
12 / 03 / 93\end{array}$ & $\begin{array}{c}\ldots . . \\
\ldots . . \\
\ldots \\
\ldots \\
\ldots \\
<. . \\
<0.002 \\
<0.002 \\
<0.002 \\
<0.002 \\
<0.002 \\
<0.002\end{array}$ & $\begin{array}{l}0.29 \\
0.47 \\
0.37 \\
0.18 \\
0.35 \\
0.09 \\
0.20 \\
0.48 \\
1.48 \\
0.30 \\
0.73 \\
0.13\end{array}$ & $\begin{array}{c}\ldots \\
\ldots \\
\ldots \\
\ldots \\
\ldots \\
0.004 \\
<0.002 \\
<0.002 \\
<0.002 \\
<0.002 \\
0.002\end{array}$ & $\begin{array}{r}<\overline{<} \\
<0.05 \\
<0.01 \\
<01 \\
0.05 \\
<0.01 \\
<0.02 \\
<0.02 \\
<0.02 \\
<0.02 \\
0.01 \\
<0.01\end{array}$ & $\begin{array}{l}1.10 \\
1.48 \\
0.87 \\
1.02 \\
0.81 \\
0.71 \\
0.88 \\
1.47 \\
0.86 \\
0.75 \\
1.98 \\
<0.01\end{array}$ & $\begin{array}{l}\ldots \\
\cdots \\
\cdots \\
\cdots \\
\cdots \\
<0.002 \\
0.002 \\
0.002 \\
0.003 \\
<0.002 \\
0.003\end{array}$ & $\begin{array}{l}<0.1 \\
<0.1 \\
<0.05 \\
<0.05 \\
<0.02 \\
<0.02 \\
<0.05 \\
<0.05 \\
0.20 \\
<0.02 \\
0.02 \\
<0.02\end{array}$ & $\begin{array}{c}- \\
\ldots \\
\ldots \\
\ldots \\
\bar{\ldots} \\
<0.002 \\
<0.002 \\
<0.002 \\
<0.002 \\
<0.002 \\
<0.002\end{array}$ & $\begin{array}{c}\ldots \\
\angle 0.10 \\
<0.10 \\
<0.10 \\
\ldots \\
\ldots \\
\ldots \\
\ldots \\
\\
<0.001\end{array}$ & 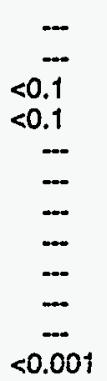 & $\begin{array}{c}-. \\
<0.1 \\
<0.1 \\
<0.1 \\
<0.1 \\
<0.1 \\
<0.1 \\
0.08 \\
0.04 \\
<0.01 \\
0.07\end{array}$ & $\begin{array}{l}- \\
- \\
-- \\
- \\
- \\
\\
<0.02 \\
<0.01 \\
<0.01 \\
<0.01 \\
<0.01\end{array}$ & $\begin{array}{l}\overline{-} \\
<0.1 \\
<0.1 \\
= \\
= \\
= \\
= \\
\overline{-}\end{array}$ & $\begin{array}{c}-. \\
0.04 \\
0.03 \\
0.01 \\
0.04 \\
0.04 \\
0.01 \\
0.02 \\
<0.01 \\
0.05 \\
0.01\end{array}$ \\
\hline $\begin{array}{l}\text { WC-3 } \\
\text { WC-19 } \\
\text { WC-33 } \\
\text { WC-42 } \\
\text { WC-57 } \\
\text { WC-77 } \\
\text { WC-79 } \\
\text { WC-99 } \\
\text { WC-107 } \\
\text { WC-127 } \\
\text { WC-140 } \\
\text { WC-152 }\end{array}$ & $\begin{array}{l}\text { Philomena's } \\
\text { Philomena's } \\
\text { Philomena's } \\
\text { Philomena's } \\
\text { Philomena's } \\
\text { Philomena's } \\
\text { Philomena's } \\
\text { Philomena's } \\
\text { Philomena's } \\
\text { Philomena's } \\
\text { Philomena's } \\
\text { Philomena's }\end{array}$ & $\begin{array}{l}05 / 23 / 90 \\
09 / 07 / 90 \\
12 / 04 / 90 \\
04 / 24 / 91 \\
08 / 05 / 91 \\
12 / 19 / 91 \\
03 / 31 / 92 \\
08 / 14 / 92 \\
12 / 03 / 92 \\
04 / 29 / 93 \\
08 / 13 / 93 \\
11 / 29 / 93\end{array}$ & $\begin{array}{l}\ldots . . \\
\ldots \\
\ldots . . \\
\ldots . . \\
\ldots . . \\
<0.002 \\
<0.002 \\
<0.002 \\
<0.002 \\
<0.002 \\
<0.002\end{array}$ & $\begin{array}{l}0.90 \\
0.42 \\
0.54 \\
0.18 \\
0.23 \\
0.17 \\
0.55 \\
0.78 \\
0.29 \\
0.27 \\
1.54 \\
0.10\end{array}$ & $\begin{array}{c}\ldots \\
\ldots \\
\ldots \\
\ldots \\
\ldots \\
0 . \\
0.023 \\
<0.002 \\
<0.002 \\
<0.002 \\
<0.002 \\
0.006\end{array}$ & $\begin{array}{r}<0.05 \\
<0.01 \\
<0.01 \\
0.05 \\
<0.01 \\
<0.02 \\
<0.02 \\
<0.02 \\
<0.02 \\
0.02 \\
<0.01\end{array}$ & $\begin{array}{l}1.46 \\
1.20 \\
0.84 \\
1.06 \\
0.65 \\
0.84 \\
1.64 \\
1.51 \\
0.93 \\
0.97 \\
2.47 \\
<0.01\end{array}$ & $\begin{array}{l}\cdots \\
\cdots \\
\cdots \\
\cdots \\
\cdots \\
<0.002 \\
<0.002 \\
0.002 \\
0.002 \\
0.002 \\
0.005\end{array}$ & $\begin{array}{c}0.17 \\
<0.10 \\
<0.05 \\
<0.05 \\
<0.02 \\
<0.02 \\
<0.05 \\
<0.05 \\
<0.05 \\
<0.02 \\
0.05 \\
<0.02\end{array}$ & $\begin{array}{l}- \\
- \\
- \\
- \\
\overline{-} \\
<0.002 \\
<0.002 \\
<0.002 \\
<0.002 \\
<0.002 \\
<0.002\end{array}$ & $\begin{array}{c}\overline{-} \\
<0.10 \\
<0.10 \\
<0.10 \\
\cdots \\
\cdots \\
\overline{-} \\
\overline{-} \\
<0.001\end{array}$ & $\begin{array}{c}\ldots \\
\cdots 0.10 \\
<0.10 \\
\ldots \\
\ldots \\
\ldots \\
\cdots \\
\cdots \\
\times 0.001\end{array}$ & $\begin{array}{c}\quad-. \\
<0.1 \\
<0.1 \\
<0.1 \\
<0.1 \\
<0.1 \\
<0.1 \\
0.08 \\
<0.01 \\
<0.01 \\
0.07\end{array}$ & $\begin{array}{l}\ldots \\
\cdots \\
\ldots \\
\cdots \\
\cdots \\
\cdots \\
<0.02 \\
<0.01 \\
<0.01 \\
<0.01 \\
<0.01\end{array}$ & $\begin{array}{c}\ldots \\
\times 0.1 \\
<0.1 \\
- \\
- \\
-- \\
\cdots \\
- \\
- \\
-\end{array}$ & $\begin{array}{l}\ldots \\
0.05 \\
0.01 \\
0.02 \\
0.02 \\
0.04 \\
0.03 \\
0.05 \\
0.06 \\
0.16 \\
0.05\end{array}$ \\
\hline $\begin{array}{l}\text { WC-4 } \\
\text { WC-20 } \\
\text { WC-34 } \\
\text { WC-45 }\end{array}$ & $\begin{array}{l}\text { Pajarito Mountain } \\
\text { Pajarito Mountain } \\
\text { Pajarito Mountain } \\
\text { Pajarito Mountain }\end{array}$ & $\begin{array}{l}05 / 23 / 90 \\
09 / 07 / 90 \\
12 / 07 / 90 \\
04 / 25 / 91\end{array}$ & $\begin{array}{l}\cdots \\
--. \\
--. \\
--.\end{array}$ & $\begin{array}{l}0.18 \\
0.92 \\
0.36 \\
0.22\end{array}$ & $\begin{array}{l}\cdots \\
\cdots \\
\cdots\end{array}$ & $\begin{array}{l}<0.05 \\
<0.01 \\
<0.01\end{array}$ & $\begin{array}{c}0.82 \\
0.85 \\
0.27 \\
<0.02\end{array}$ & $m$ & $\begin{array}{l}<0.1 \\
0.23 \\
1.23 \\
0.23\end{array}$ & $m$ & $\begin{array}{l}\ldots . . \\
<0.1 \\
<0.1\end{array}$ & $\begin{array}{l}\ldots . . \\
<0.1 \\
<0.1\end{array}$ & $\begin{array}{c}\ldots \\
<0.1 \\
0.1\end{array}$ & $m$ & $\begin{array}{l}-\infty \\
<0.1 \\
<0.1\end{array}$ & $\begin{array}{l}\ldots \\
0.12 \\
0.05\end{array}$ \\
\hline
\end{tabular}




\begin{tabular}{|c|c|c|c|c|c|c|c|c|c|c|c|c|c|c|c|}
\hline Sample \# & Description & Date & $\begin{array}{c}\mathrm{Ag} \\
\mathrm{mg} / \mathrm{kg}\end{array}$ & $\begin{array}{c}\mathrm{Al} \\
\mathrm{mg} / \mathrm{kg}\end{array}$ & $\begin{array}{c}\text { As } \\
\mathrm{mg} / \mathrm{kg}\end{array}$ & $\begin{array}{c}\mathrm{Ba} \\
\mathrm{mg} / \mathrm{kg}\end{array}$ & $\begin{array}{c}\mathrm{Cd} \\
\mathrm{mg} / \mathrm{kg}\end{array}$ & $\begin{array}{c}\mathrm{Co} \\
\mathrm{mg} / \mathrm{kg}\end{array}$ & $\begin{array}{c}\mathrm{Cr} \\
\mathrm{mg} / \mathrm{kg}\end{array}$ & $\begin{array}{c}\mathrm{Cs} \\
\mathrm{mg} / \mathrm{kg}\end{array}$ & $\begin{array}{c}\mathrm{Cu} \\
\mathrm{mg} / \mathrm{kg}\end{array}$ & $\begin{array}{c}\mathrm{Fe} \\
\mathrm{mg} / \mathrm{kg}\end{array}$ & $\begin{array}{c}\mathrm{Hg} \\
\mathrm{mg} / \mathrm{kg}\end{array}$ & $\begin{array}{c}\mathrm{I} \\
\mathrm{mg} / \mathrm{kg}\end{array}$ & $\begin{array}{c}\mathrm{Mn} \\
\mathrm{mg} / \mathrm{kg}\end{array}$ \\
\hline WC-61 & Pajarito Mountain & $08 / 06 / 91$ & $\ldots$ & $<0.1$ & $<0.05$ & $<0.02$ & $\ldots$ & - & $\cdots$ & - & $\ldots$ & 0.02 & $\ldots$ & $\Longrightarrow$ & $<0.01$ \\
\hline WC-69 & Pajarito Mountain & $12 / 08 / 91$ & $\ldots$ & $<0.1$ & $<0.05$ & $<0.01$ & ... & $\ldots$ & --. & $-\cdots$ & $-\cdots$ & $<0.01$ & $\ldots$ & $\ldots$ & $<0.01$ \\
\hline WC-90 & Pajarito Mountain & $05 / 07 / 92$ & $<0.001$ & $<0.1$ & $<0.05$ & $<0.01$ & $<0.001$ & $<0.002$ & $<0.002$ & $<0.002$ & 0.002 & $<0.01$ & - & - & $<0.01$ \\
\hline WC-93 & Pajarito Mountain & $08 / 10 / 92$ & $<0.001$ & $<0.1$ & $<0.05$ & $<0.01$ & $<0.001$ & $<0.002$ & $<0.002$ & $<0.002$ & 0.003 & 0.01 & $\ldots$ & $<0.02$ & $<0.01$ \\
\hline WC-116 & Pajarito Mountain & $12 / 16 / 92$ & $<0.001$ & $<0.1$ & $<0.05$ & $<0.01$ & $<0.001$ & $<0.002$ & $<0.002$ & 0 & 0.01 & $<0.01$ & - & $<0.01$ & $<0.01$ \\
\hline WC-130 & Pajarito Mountain & $05 / 04 / 93$ & $<0.001$ & $<0.1$ & $<0.05$ & $<0.01$ & 0.003 & $<0.002$ & $<0.002$ & 0 & $<0.002$ & $<0.01$ & .-. & $<0.01$ & $<0.01$ \\
\hline WC-136 & Pajarito Mountain & $08 / 09 / 93$ & $<0.0005$ & $<0.1$ & 0.11 & $<0.01$ & 0.0017 & $<0.002$ & $<0.002$ & $<0.002$ & & 0.04 & --- & $<0.01$ & $<0.01$ \\
\hline WC-155 & Pajarito Mountain & $02 / 02 / 94$ & $<0.0005$ & 0.07 & 0.001 & 0.03 & 0.0079 & 0.003 & $<0.002$ & $<0.002$ & 0.02 & 0.03 & $<0.0005$ & $<0.01$ & 0.02 \\
\hline WC-5 & "S" Site & $05 / 30 / 90$ & $\ldots$ & $\cdots$ & $\cdots$ & $\cdots$ & -- & $\ldots$ & -- & -- & $\cdots$ &.- & $\cdots$ & $\cdots$ & $\ldots$ \\
\hline WC-15 & "S" Site & $9 / 05 / 90$ & $\ldots$ & $\ldots$ & ... & $\ldots$ & ... & $\ldots$ & ... & $\ldots$ & $\ldots$ & $\ldots$ & $\ldots$ & $\ldots$ & $\ldots$ \\
\hline WC-31 & "S" Site & $12 / 04 / 90$ & -.- & $<0.1$ & $<0.05$ & $<0.01$ & --- & $\ldots$ & $\ldots$ & $\ldots$ & $\cdots$ & $<0.01$ & $<0.1$ & - & $<0.01$ \\
\hline WC- 41 & "S" Site & $04 / 24 / 91$ & $\ldots$ & $<0.1$ & 0.05 & $<0.01$ & ... & ... & $-\cdots$ & --. & --- & $<0.01$ & $<0.1$ & --- & $<0.01$ \\
\hline WC-56 & "S" Site & $08 / 05 / 91$ & -- & $<0.1$ & 0.05 & $<0.02$ & ... & -. & ... & $\ldots$ & -.. & 0.03 & -.- & --- & $<0.01$ \\
\hline WC-78 & "S" Site & $12 / 19 / 91$ & $\ldots$ & $<0.1$ & $<0.05$ & $<0.01$ & --- & $\ldots$ & -.. & --- & -- & $<0.01$ & --. & $\ldots$ & 0 \\
\hline WC-87 & "S" Site & $04 / 29 / 92$ & $<0.001$ & $<0.1$ & $<0.05$ & $<0.01$ & 0.001 & $<0.002$ & $<0.002$ & $<0.002$ & 0.004 & $<0.01$ & $\cdots$ & $\ldots$ & $<0.01$ \\
\hline WC-103 & "S" Site. & $08 / 20 / 92$ & $<0.001$ & $<0.1$ & $<0.05$ & $<0.01$ & $<0.001$ & $<0.002$ & $<0.002$ & 0.01 & 0.002 & $<0.01$ & $\ldots$ & $<0.02$ & $<0.01$ \\
\hline WC- 115 & "S" Site & $12 / 16 / 92$ & $<0.001$ & $<0.1$ & $<0.05$ & $<0.01$ & $<0.001$ & $<0.002$ & $<0.002$ & $<0.002$ & & $<0.01$ & $\ldots$ & $<0.01$ & $<0.01$ \\
\hline WC-129 & "S" Site & $04 / 30 / 93$ & $<0.001$ & $<0.1$ & $<0.05$ & $<0.01$ & 0.001 & $<0.002$ & $<0.002$ & $<0.002$ & 0.007 & 0.02 & $\cdots$ & $<0.01$ & $<0.01$ \\
\hline WC-134 & "S" Site & $08 / 05 / 93$ & $<0.0005$ & $<0.1$ & $<0.05$ & $<0.01$ & 0.0003 & $<0.002$ & 0.005 & $<0.002$ & 0 & 0.04 & -.. & $<0.01$ & $<0.01$ \\
\hline WC-153 & "S" Site & $11 / 30 / 93$ & $<0.0005$ & $<0.05$ & $<0.0005$ & $<0.01$ & 0.0006 & $<0.002$ & $<0.002$ & $<0.002$ & 0.02 & 0.01 & $=0.0005$ & $<0.01$ & $<0.01$ \\
\hline WC-6 & White Rock "Y" & $05 / 5$ & $\ldots$ & $\ldots$ & $\ldots$ & $\ldots$ & ..- & $\ldots$ & --- & --. & --- & ... & -.. & ... & -- \\
\hline WC-10 & White Rock "Y" & $08 / 0$ & -- & ... & ... & $\ldots$ & -- & ... & ..- & -- & --. & --. & -- & --- & -- \\
\hline WC-32 & White Rock "Y" & $12 / 04 / 90$ & --- & $<0.1$ & $<0.05$ & $<0.01$ & $\ldots$ & $\ldots$ & $\ldots$ & $\ldots$ & $\ldots$ & $<0.01$ & $<0.1$ & ...- & 0.04 \\
\hline WC-43 & White Rock "Y" & $04 / 24 / 91$ & $\ldots$ & $<0.1$ & $<0.05$ & $<0.01$ & --. & $\ldots$ & -.- & $\cdots$ & --- & $<0.01$ & $<0.1$ & $\cdots$ & $<0.01$ \\
\hline WC-55 & White Rock "Y" & $08 / 01 / 91$ & $\ldots$ & $<0.1$ & $<0.05$ & $<0.02$ & $\ldots$ & ... & ... & -.. & -- & $<0.02$ & $\ldots$ & -.- & $<0.01$ \\
\hline WC-72 & White Rc & $12 / 10 / 91$ & .- & $<0.1$ & $<0.05$ & $<0.01$ & $\ldots$ & $\ldots$ & $\ldots$ & $\ldots$ & ... & $<0.01$ & $\ldots$ & $\ldots$ & $<0.01$ \\
\hline WC- 84 & White Rock "Y" & $04 / 28 / 92$ & $<0.001$ & $<0.1$ & $<0.05$ & $<0.01$ & 0.004 & $<0.002$ & $<0.002$ & $<0.002$ & 0.003 & $<0.01$ & $\ldots$ & $\ldots$ & $<0.01$ \\
\hline WC-98 & White Rock "Y" & $08 / 14 / 92$ & $<0.001$ & $<0.1$ & $<0.05$ & 0.03 & 0.003 & $<0.002$ & $<0.002$ & $<0.002$ & 0.009 & 0.04 & $\ldots$ & $<0.02$ & $<0.01$ \\
\hline WC- 108 & White Rock "Y" & $12 / 03 / 92$ & $<0.001$ & $<0.1$ & $<0.05$ & $<0.01$ & $<0.001$ & $<0.002$ & $<0.002$ & $<0.002$ & 0.005 & $<0.01$ & --- & $<0.01$ & $<0.01$ \\
\hline WC-126 & White Rock "Y" & $04 / 29 / 93$ & $<0.001$ & $<0.1$ & $<0.05$ & $<0.01$ & $<0.001$ & $<0.002$ & 0.002 & $<0.002$ & 0.005 & 0.02 & $\ldots$ & $<0.01$ & $<0.01$ \\
\hline WC-137 & White Rock "Y" & $08 / 09 / 93$ & $<0.0005$ & $<0.1$ & $<0.05$ & 0.01 & 0.0054 & $<0.002$ & 0.003 & $<0.002$ & 0.005 & 0.03 & --. & $<0.01$ & $<0.01$ \\
\hline WC- 148 & White Rock "Y" & $11 / 29 / 93$ & $<0.0005$ & $<0.05$ & 0.0006 & $<0.01$ & $<0.0005$ & $<0.002$ & $<0.002$ & $<0.002$ & 0.002 & 0.01 & $<0.0005$ & $<0.01$ & $<0.01$ \\
\hline WC- & Stat & 0 & $\ldots$ & $\ldots$ & ... & ... & $\ldots$ & ... & ... & ..- & ... & $\ldots$ & .-. & ... & ..- \\
\hline WC-14 & Espanola Ranger Station & $09 / 05 / 90$ & ..- & $\ldots$ & ..- & ..- & - & -.- & ..- & -- & $\ldots$ & -.. & -.. & $\ldots$ & --. \\
\hline WC-30 & Espanola Ranger Station & $11 / 28 / 90$ & $\ldots$ & $<0.1$ & $<0.05$ & $<0.01$ & $\ldots$ & ... & ... & $\ldots$ & $\ldots$ & $<0.01$ & $<0.1$ & ... & $<0.01$ \\
\hline WC-44 & panola Ranger Station & $04 / 24 / 91$ & $\ldots$ & $<0.1$ & $<0.05$ & $<0.01$ & ..- & $\ldots$ & $\ldots$ & $\ldots$ & ... & $<0.01$ & $<0.1$ & m.. & $<0.01$ \\
\hline WC-58 & Espanola Ranger Station & $08 / 05 / 91$ & $\ldots$ & $<0.1$ & $<0.05$ & $<0.02$ & -.. & -.. & .-- & $\ldots$ & ... & $<0.02$ & $\ldots$ & $\ldots$ & $<0.01$ \\
\hline WC-71 & Espanola Ranger Station & $12 / 10 / 91$ & -.. & $<0.1$ & $<0.05$ & $<0.01$ & $\ldots$ & $\ldots$ & -- & -- & --- & $<0.01$ & $\cdots$ & --- & $<0.01$ \\
\hline WC- 86 & Espanola Ranger Station & $04 / 29 / 92$ & $<0.001$ & $<0.1$ & $<0.05$ & $<0.01$ & 0.001 & $<0.002$ & $<0.002$ & $<0.002$ & 0.007 & $<0.01$ & --- & -.- & $<0.01$ \\
\hline WC-101 & Espanola Ranger Station & $08 / 20 / 92$ & $<0.001$ & $<0.1$ & $<0.05$ & $<0.01$ & $<0.001$ & $<0.002$ & $<0.002$ & $<0.002$ & 0.005 & $<0.01$ & -.. & $<0.02$ & $<0.01$ \\
\hline WC-114 & Espanola Ranger Station & $12 / 16 / 92$ & $<0.001$ & $<0.1$ & $<0.05$ & $<0.01$ & $<0.001$ & $<0.002$ & $<0.002$ & $<0.002$ & 0.007 & $<0.01$ & $\cdots$ & $<0.01$ & $<0.01$ \\
\hline WC-128 & Espanola Ranger Station & $04 / 30 / 93$ & $<0.001$ & $<0.1$ & $<0.05$ & $<0.01$ & 0.001 & $<0.002$ & $<0.002$ & $<0.002$ & 0.002 & $<0.01$ & $\cdots$ & $<0.01$ & $<0.01$ \\
\hline
\end{tabular}




\begin{tabular}{|c|c|c|c|c|c|c|c|c|c|c|c|c|c|c|c|c|}
\hline Sample \# & Description & Date & $\begin{array}{c}\mathrm{Mo} \\
\mathrm{mg} / \mathrm{kg}\end{array}$ & $\begin{array}{l}\mathrm{NH4} \\
\mathrm{mg} / \mathrm{kg}\end{array}$ & $\begin{array}{l}\mathrm{Ni} \\
\mathrm{mg} / \mathrm{kg}\end{array}$ & $\begin{array}{l}\mathrm{NO} 2 \\
\mathrm{mg} / \mathrm{kg}\end{array}$ & $\begin{array}{l}\mathrm{NO3} \\
\mathrm{mg} / \mathrm{kg}\end{array}$ & $\begin{array}{l}\mathrm{Pb} \\
\mathrm{mg} / \mathrm{kg}\end{array}$ & $\begin{array}{l}\mathrm{PO} 4 \\
\mathrm{mg} / \mathrm{kg}\end{array}$ & $\begin{array}{c}\mathrm{Ab} \\
\mathrm{mg} / \mathrm{kg}\end{array}$ & $\begin{array}{l}\mathrm{Sb} \\
\mathrm{mg} / \mathrm{kg}\end{array}$ & $\begin{array}{l}\mathrm{Se} \\
\mathrm{mg} / \mathrm{kg}\end{array}$ & $\begin{array}{l}\mathrm{Si} \\
\mathrm{mg} / \mathrm{kg}\end{array}$ & $\begin{array}{l}\mathrm{S} 2 \mathrm{O} 3 \\
\mathrm{mg} / \mathrm{kg}\end{array}$ & $\underset{\mathrm{mg} / \mathrm{kg}}{\mathrm{U}}$ & $\begin{array}{l}\mathrm{Zn} \\
\mathrm{mg} / \mathrm{kg}\end{array}$ \\
\hline WC-61 & Pajarito Mountain & $08 / 06 / 91$ & $\cdots$ & 0.86 & $\ldots$ & 0.06 & 0.61 & $\ldots$ & 0.34 & $\ldots$ & $<0.1$ & - & $<0.1$ & $\ldots$ & $\cdots$ & 1.08 \\
\hline WC-69 & Pajarito Mountain & $12 / 08 / 91$ & ... & 0.77 & ... & $<0.01$ & 0.40 & - & $<0.02$ & ... & - & $\ldots$ & $<0.1$ & $\ldots$ & - & 0.07 \\
\hline WC-90 & Pajarito Mountain & $05 / 07 / 92$ & $<0.002$ & 0.29 & 0.01 & $<0.02$ & 0.66 & $<0.002$ & $<0.05$ & $<0.002$ & -.- & -- & $<0.1$ & .- & -- & 0.03 \\
\hline WC-93 & Pajarito Mountain & $08 / 10 / 92$ & $<0.002$ & 0.66 & $<0.002$ & $<0.02$ & 1.10 & $<0.002$ & $<0.05$ & $<0.002$ & $\ldots$ & $\ldots$ & $<0.1$ & $<0.02$ & $\ldots$ & 0.01 \\
\hline WC-116 & Pajarito Mountain & $12 / 16 / 92$ & $<0.002$ & 0.50 & $<0.002$ & $<0.02$ & 0.85 & 0.003 & $<0.05$ & $<0.002$ & $\ldots$ & -. & 0.07 & $<0.01$ & $\ldots$ & 0.02 \\
\hline WC-130 & Pajarito Mountain & $05 / 04 / 93$ & $<0.002$ & 0.36 & $<0.002$ & $<0.02$ & 0.92 & 0.004 & $<0.02$ & $<0.002$ & - & - & $<0.01$ & $<0.01$ & - & $<0.01$ \\
\hline WC-136 & Pajarito Mountain & $08 / 09 / 93$ & $<0.002$ & 2.06 & $<0.002$ & 0.18 & 1.79 & 0.004 & 0.29 & $<0.002$ & - & - & $<0.01$ & $<0.01$ & -- & 0.09 \\
\hline WC-155 & Pajarito Mountain & $02 / 02 / 94$ & $<0.002$ & 0.38 & 0.11 & $<0.02$ & 1.71 & 0.007 & $<0.02$ & $<0.002$ & $<0.001$ & $<0.001$ & $<0.1$ & $<0.01$ & $\cdots$ & 0.09 \\
\hline WC-5 & "S" Site & $05 / 30 / 90$ & $\cdots$ & 1.61 & $\ldots$ & - & 1.37 & $\ldots$ & 0.45 & ... & ... & -- & ... & -. & -. & - \\
\hline WC-15 & "S" Site & $09 / 05 / 90$ & ... & 3.10 & $\ldots$ & 20.9 & 8.33 & ..- & 3.69 & $\ldots$ & $\ldots$ & - & $\ldots$ & $\ldots$ & $\ldots$ & $\ldots$ \\
\hline WC-31 & "S" Site & $12 / 04 / 90$ & ... & 1.31 & -.- & 0.05 & 1.45 & -.. & 0.17 & -.. & $<0.1$ & $<0.1$ & $<0.1$ & $\ldots$ & $<0.1$ & 0.37 \\
\hline WC-41 & "S" Site & $04 / 24 / 91$ & -.. & 0.16 & -.. & $<0.01$ & 0.89 & -.- & $<0.05$ & -.. & $<0.1$ & $<0.1$ & $<0.1$ & $\ldots$ & $<0.1$ & 0.02 \\
\hline WC-56 & "S" Site & $08 / 05 / 91$ & --. & 0.19 & --. & 0.06 & 0.64 & -.- & $<0.02$ & $\ldots$ & $<0.1$ & $\ldots$ & $<0.1$ & - & $\ldots$ & 0.05 \\
\hline WC-78 & "S" Site & $12 / 19 / 91$ & $\ldots$ & 0.28 & - & $<0.01$ & 0.76 & $\ldots$ & $<0.02$ & $\cdots$ & $\ldots$ & - & $<0.1$ & -. & -. & 0.03 \\
\hline WC-87 & "S" Site & $04 / 29 / 92$ & $<0.002$ & 0.38 & 0.006 & $<0.02$ & 1.22 & $<0.002$ & $<0.05$ & $<0.002$ & $\ldots$ & $\ldots$ & $<0.1$ & -. & - & 0.05 \\
\hline WC-103 & "S" Site & 08/20/92 & $<0.002$ & 0.22 & $<0.002$ & $<0.02$ & 1.09 & 0.002 & $<0.05$ & $<0.002$ & $\ldots$ & $\ldots$ & $<0.1$ & $<0.02$ & $\ldots$ & 0.06 \\
\hline WC-115 & "S" Site & $12 / 16 / 92$ & $<0.002$ & 0.25 & $<0.002$ & $<0.02$ & 0.27 & 0.002 & $<0.05$ & $<0.002$ & $\ldots$ & $\ldots$ & 0.05 & $<0.01$ & $\ldots$ & 0.02 \\
\hline WC-129 & "S" Site & $04 / 30 / 93$ & $<0.002$ & 0.30 & $<0.002$ & 0.02 & 0.75 & $<0.002$ & $<0.02$ & $<0.002$ & $\ldots$ & - & $<0.01$ & $<0.01$ & -.. & $<0.01$ \\
\hline WC-134 & "S" Site & $08 / 05 / 93$ & $<0.002$ & 0.48 & $<0.002$ & 0.01 & 1.54 & $<0.002$ & 0.02 & $<0.002$ & -.. & - & $<0.01$ & $<0.01$ & $\ldots$ & 0.09 \\
\hline WC-153 & "S" Site & $11 / 30 / 93$ & $<0.002$ & 0.44 & 0.01 & 0.04 & 0.92 & 0.007 & $<0.02$ & $<0.002$ & $<0.001$ & $<0.001$ & 0.05 & $<0.01$ & - & 0.07 \\
\hline WC-6 & White Rock "Y" & $05 / 30 / 90$ & $\ldots$ & 0.05 & $\ldots$ & $\ldots$ & 1.35 & ..- & $<0.10$ & -.- & -.. & $\ldots$ & -.- & - & - & $\ldots$ \\
\hline WC-10 & White Rock "Y" & $08 / 09 / 90$ & -.- & 0.20 & $\ldots$ & $<0.05$ & 1.28 & $\ldots$ & $<0.10$ & ... & $\ldots$ & ... & ..- & -- & -.. & --. \\
\hline WC-32 & While Rock "Y" & $12 / 04 / 90$ & $\ldots$ & 1.58 & $\ldots$ & $<0.01$ & 5.09 & $\ldots$ & 0.40 & $\ldots$ & $<0.1$ & $<0.1$ & $<0.1$ & --- & $<0.1$ & 0.35 \\
\hline$w C-43$ & White Rock "Y" & $04 / 24 / 91$ & $\ldots$ & 0.17 & $\ldots$ & $<0.01$ & 1,10 & $\ldots$ & $<0.05$ & $\ldots$ & $<0.1$ & $<0.1$ & $<0.1$ & -.. & $<0.1$ & 0.07 \\
\hline WC-55 & White Rock "Y" & $08 / 01 / 91$ & ... & 0.19 & ... & 0.04 & 0.59 & $\ldots$ & $<0.02$ & -.. & $<0.1$ & - & $<0.1$ & $\ldots$ & $\ldots$ & 0.06 \\
\hline WC-72 & White Rock "Y" & $12 / 10 / 91$ & ..- & 0.13 & $\ldots$ & $<0.01$ & 0.55 & -- & $<0.02$ & --. & $\cdots$ & $\ldots$ & $<0.1$ & $\therefore$ & - & 0.07 \\
\hline WC-84 & White Rock "Y" & $04 / 28 / 92$ & $<0.002$ & 0.17 & 0.004 & $<0.02$ & 1.02 & $<0.002$ & $<0.05$ & $<0.002$ & ... & $\ldots$ & $<0.1$ & -- & $\ldots$ & 0.37 \\
\hline WC-98 & White Rock "Y" & $08 / 14 / 92$ & $<0.002$ & 0.88 & 0.004 & $<0.02$ & 1.39 & 0.002 & $<0.05$ & $<0.002$ & $\ldots$ & -- & $<0.1$ & $<0.02$ & $-\infty$ & 0.11 \\
\hline WC-108 & White Rock "Y" & $12 / 03 / 92$ & $<0.002$ & 0.24 & $<0.002$ & $<0.02$ & 1.13 & 0.002 & $<0.05$ & $<0.002$ & $\ldots$ & $\ldots$ & 0.09 & $<0.01$ & $\ldots$ & 0.12 \\
\hline WC-126 & White Rock "Y" & $04 / 29 / 93$ & $<0.002$ & 0.25 & $<0.002$ & $<0.02$ & 0.79 & 0.008 & $<0.02$ & $<0.002$ & $\ldots$ & - & 0.01 & $<0.01$ & -.. & 0.03 \\
\hline WC-137 & White Rock "Y" & $08 / 09 / 93$ & 0.002 & 0.24 & 0.002 & $<0.01$ & 2.51 & 0.003 & $<0.02$ & 0.002 & $\ldots$ & $\ldots$ & $<0.01$ & $<0.01$ & - & 0.30 \\
\hline WC-148 & White Rock "Y" & $11 / 29 / 93$ & $<0.002$ & 0.30 & 0.003 & $<0.01$ & 0.89 & 0.006 & $<0.02$ & $<0.002$ & $<0.001$ & $<0.001$ & 0.04 & $<0.01$ & $-\infty$ & 0.09 \\
\hline C-7 & Espanola Ranger Station & $05 / 30 / 90$ & $\ldots$ & 0.26 & $\cdots$ & $\ldots$ & 1.77 & -- & 0.15 & $\cdots$ & $\cdots$ & $\cdots$ & $\ldots$ & -.. & - & - \\
\hline WC-14 & Espanola Ranger Station & $09 / 05 / 90$ & $\ldots$ & 0.09 & $\ldots$ & $<0.05$ & 1.72 & $\ldots$ & $<0.10$ & -.. & ... & $\ldots$ & $\ldots$ & $\ldots$ & $\ldots$ & --- \\
\hline WC-30 & Espanola Ranger Station & $11 / 28 / 90$ & ..- & 0.18 & .-- & 0.05 & 0.87 & -.. & $<0.05$ & -.. & $<0.1$ & $<0.1$ & $<0.1$ & -- & $<0.1$ & 0.97 \\
\hline WC-44 & Espanola Ranger Station & $04 / 24 / 91$ & $\ldots$ & 0.21 & -- & 0.09 & 2.25 & $\ldots$ & $<0.05$ & -.. & $<0.1$ & $<0.1$ & $<0.1$ & -.- & $<0.1$ & 0.10 \\
\hline WC-58 & Espanola Ranger Station & $08 / 05 / 91$ & ... & 0.16 & ב. & 0.33 & 0.84 & $\ldots$ & $<0.02$ & ..- & $<0.1$ & -. & $<0.1$ & $\ldots$ & ... & 0.56 \\
\hline WC-71 & Espanola Ranger Station & $12 / 10 / 91$ & ..- & 0.18 & - & $<0.01$ & 0.95 & $\ldots$ & $<0.02$ & -.- & $\ldots$ & $\ldots$ & $<0.1$ & -.. & - & 0.02 \\
\hline WC-86 & Espanola Ranger Station & $04 / 29 / 92$ & $<0.002$ & 0.44 & 0.008 & 0.03 & 1.31 & $<0.002$ & $<0.05$ & $<0.002$ & -. & - & $<0.1$ & -.. & - & 0.03 \\
\hline WC-101 & Espanola Ranger Station & $08 / 20 / 92$ & $<0.002$ & 0.72 & $<0.002$ & $<0.02$ & 1.70 & 0.003 & $<0.05$ & $<0.002$ & $\ldots$ & -. & $<0.1$ & $<0.02$ & - & 0.03 \\
\hline WC-114 & Espanola Ranger Station & $12 / 16 / 92$ & $<0.002$ & 0.46 & 0.003 & $<0.02$ & 0.93 & 0.003 & $<0.05$ & $<0.002$ & $\ldots$ & -. & 0.11 & $<0.01$ & - & 0.03 \\
\hline WC-128 & Espanola Ranger Station & $04 / 30 / 93$ & $<0.002$ & 0.35 & $<0.002$ & 0.03 & 1.33 & $<0.002$ & 0.09 & $<0.002$ & - & -- & 0.09 & $<0.01$ & -. & $<0.01$ \\
\hline
\end{tabular}




\begin{tabular}{|c|c|c|c|c|c|c|c|c|c|c|c|c|c|c|c|}
\hline Sample\# & Description & Date & $\begin{array}{c}\mathrm{Ag} \\
\mathrm{mg} / \mathrm{kg}\end{array}$ & $\begin{array}{r}\mathrm{Al} \\
\mathrm{mg} / \mathrm{kg}\end{array}$ & $\begin{array}{c}\text { As } \\
\mathrm{mg} / \mathrm{kg}\end{array}$ & $\begin{array}{c}\mathrm{Ba} \\
\mathrm{mg} / \mathrm{kg}\end{array}$ & $\begin{array}{c}\mathrm{Cd} \\
\mathrm{mg} / \mathrm{kg}\end{array}$ & $\begin{array}{c}\mathrm{Co} \\
\mathrm{mg} / \mathrm{kg}\end{array}$ & $\begin{array}{c}\mathrm{Cr} \\
\mathrm{mg} / \mathrm{kg}\end{array}$ & $\begin{array}{c}\mathrm{Cs} \\
\mathrm{mg} / \mathrm{kg}\end{array}$ & $\begin{array}{c}\mathrm{Cu} \\
\mathrm{mg} / \mathrm{kg}\end{array}$ & $\begin{array}{c}\mathrm{Fe} \\
\mathrm{mg} / \mathrm{kg}\end{array}$ & $\begin{array}{c}\mathrm{Hg} \\
\mathrm{mg} / \mathrm{kg}\end{array}$ & $\begin{array}{c}1 \\
\mathrm{mg} / \mathrm{kg}\end{array}$ & $\underset{\mathrm{mg} / \mathrm{kg}}{\mathrm{Mn}}$ \\
\hline $\begin{array}{l}\text { WC-133 } \\
\text { WC-147 }\end{array}$ & $\begin{array}{l}\text { spanola Ranger Station } \\
\text { spanola Ranger Station }\end{array}$ & $\begin{array}{l}8 / 05 / 93 \\
11 / 29 / 93\end{array}$ & $\begin{array}{l}<0.0005 \\
<0.0005\end{array}$ & $\begin{array}{l}<0.1 \\
<0.05\end{array}$ & $\begin{array}{l}<0.05 \\
<0.0005\end{array}$ & $\begin{array}{r}0.02 \\
<0.01\end{array}$ & $\begin{array}{l}<0.0002 \\
<0.0005\end{array}$ & $\begin{array}{l}<0.002 \\
<0.002\end{array}$ & $\begin{array}{l}<0.002 \\
<0.002\end{array}$ & $\begin{array}{l}<0.002 \\
<0.002\end{array}$ & $\begin{array}{l}0.006 \\
0.007\end{array}$ & $\begin{array}{r}0.02 \\
<0.01\end{array}$ & $<0.0005$ & $\begin{array}{l}<0.01 \\
<0.01\end{array}$ & $\begin{array}{l}<0.01 \\
<0.01\end{array}$ \\
\hline $\begin{array}{l}\text { WC-11 } \\
\text { WC-23 } \\
\text { WC-36 } \\
\text { WC-49 } \\
\text { WC-94 } \\
\text { WC-124 } \\
\text { WC-131 } \\
\text { WC-143 }\end{array}$ & $\begin{array}{l}\text { Boundary Peak Area } \\
\text { Boundary Peak Area } \\
\text { Boundary Peak Area } \\
\text { Boundary Peak Area } \\
\text { Boundary Peak Area } \\
\text { Boundary Peak Area } \\
\text { Boundary Peak Area } \\
\text { Boundary Peak Area }\end{array}$ & $\begin{array}{l}9 / 04 / 90 \\
1 / 26 / 90 \\
4 / 23 / 91 \\
7 / 29 / 91 \\
8 / 10 / 92 \\
4 / 28 / 93 \\
8 / 03 / 93 \\
1 / 16 / 93\end{array}$ & $\begin{array}{l}\cdots \\
\cdots \\
\cdots \\
<0.001 \\
<0.001 \\
<0.0005 \\
<0.0005\end{array}$ & $\begin{array}{l}<0.1 \\
<0.1 \\
<0.1 \\
<0.1 \\
<0.1 \\
<0.1 \\
<0.05\end{array}$ & $\begin{array}{l}. .05 \\
.05 \\
.05 \\
.05 \\
.05 \\
.05 \\
.0012\end{array}$ & $\begin{array}{c}<0.01 \\
<0.01 \\
<0.02 \\
<0.01 \\
0.02 \\
0.01 \\
<0.01\end{array}$ & $\begin{array}{c}\ldots \\
\cdots \\
\cdots \\
<0.001 \\
<0.001 \\
0.0003 \\
<0.0005\end{array}$ & $\begin{array}{c}\ldots \\
\cdots \\
\cdots \\
<0.002 \\
<0.002 \\
<0.002 \\
<0.002\end{array}$ & $\begin{array}{c}\cdots \\
\cdots \\
\cdots \\
<0.002 \\
<0.002 \\
0.002 \\
<0.002\end{array}$ & $\begin{array}{c}\ldots \\
\cdots \\
\cdots- \\
<0.002 \\
0.010 \\
<0.002 \\
<0.002\end{array}$ & $\begin{array}{c}\cdots \\
\cdots \\
\cdots \\
<0.002 \\
0.010 \\
0.012 \\
0.010\end{array}$ & $\begin{array}{r}<\overline{0.01} \\
<0.01 \\
<0.02 \\
<0.01 \\
0.08 \\
0.10 \\
<0.01\end{array}$ & $\begin{array}{c}<0 . \overline{1} \\
<0.1 \\
\ldots \\
\ldots \\
\ldots \\
\cdots \\
<0.0005\end{array}$ & $\begin{array}{c}\cdots \\
\cdots \\
\cdots \\
<0.02 \\
<0.01 \\
<0.01 \\
<0.01\end{array}$ & $\begin{array}{l}<0.01 \\
<0.01 \\
<0.01 \\
<0.01 \\
<0.01 \\
<0.01 \\
<0.01\end{array}$ \\
\hline $\begin{array}{l}9 \\
0 \\
6 \\
5 \\
2 \\
06 \\
19 \\
35 \\
44\end{array}$ & $\begin{array}{l}\text { Atn. } \\
\text { Atn. } \\
\text { Atn. } \\
\text { Atn. } \\
\text { Atn. } \\
\text { Atn. } \\
\text { Atn. } \\
\text { Atn. } \\
\text { Atn. } \\
\text { Atn. }\end{array}$ & $\begin{array}{l}30 \\
31 \\
31 \\
31 \\
32 \\
92 \\
2 \\
93 \\
33 \\
33\end{array}$ & $\begin{array}{c}\ldots \\
\ldots . . \\
\ldots \\
\ldots \\
\ldots . . \\
<0.001 \\
<0.001 \\
<0.001 \\
<0.001 \\
<0.0005\end{array}$ & $\begin{array}{l}<0.1 \\
<0.1 \\
<0.1 \\
<0.1 \\
<0.1 \\
<0.1 \\
<0.1 \\
<0.1 \\
<0.1 \\
<0.05\end{array}$ & $\begin{array}{l}. . \\
.05 \\
.05 \\
.05 \\
.05 \\
.05 \\
.05 \\
.05 \\
.05 \\
.05 \\
.0005\end{array}$ & $\begin{array}{l}<0.01 \\
<0.01 \\
<0.02 \\
<0.01 \\
<0.01 \\
<0.01 \\
<0.01 \\
<0.01 \\
<0.01 \\
<0.01\end{array}$ & $\begin{array}{c}\ldots . . \\
\ldots . \\
\ldots . \\
\ldots . \\
0.003 \\
0.001 \\
0.001 \\
0.001 \\
0.0003\end{array}$ & $\begin{array}{c}\ldots \\
\ldots \\
\ldots \\
\ldots \\
\ldots 0 . \\
<0.02 \\
<0.002 \\
<0.002 \\
<0.002 \\
<0.002 \\
<0.002\end{array}$ & $\begin{array}{c}\ldots \\
-. . \\
-- \\
-- \\
<0.002 \\
0.003 \\
<0.002 \\
<0.002 \\
<0.002 \\
<0.002\end{array}$ & $\begin{array}{c}\ldots \\
\cdots \\
\cdots \\
\ldots . \\
0 . . \\
0.004 \\
<0.002 \\
<0.002 \\
<0.002 \\
<0.002 \\
<0.002\end{array}$ & $\begin{array}{l}\ldots \\
\ldots . \\
\ldots \\
\ldots- \\
\ldots . \\
.011 \\
.008 \\
.005 \\
.008 \\
.009 \\
.010\end{array}$ & $\begin{array}{r}<\overline{-} \\
<0.01 \\
<0.01 \\
<0.02 \\
0.03 \\
<0.01 \\
0.04 \\
<0.01 \\
<0.01 \\
0.06 \\
0.03\end{array}$ & $\begin{array}{c}<0.1 \\
<0.1 \\
\ldots \\
\cdots \\
\cdots \\
\cdots \\
\cdots \\
\cdots \\
<0.0005\end{array}$ & $\begin{array}{c}\cdots \\
\cdots-. \\
-- \\
\cdots- \\
\cdots- \\
<0.02 \\
<0.01 \\
<0.01 \\
<0.01 \\
<0.01\end{array}$ & $\begin{aligned} &- \\
&<0.01 \\
&<0.01 \\
&<0.01 \\
& 0.02 \\
&<0.01 \\
&<0.01 \\
&<0.01 \\
&<0.01 \\
&<0.01 \\
&<0.01\end{aligned}$ \\
\hline $\begin{array}{l}\text { WC-29 } \\
\text { WC-47 } \\
\text { WC-52 } \\
\text { WC-66 } \\
\text { WC-81 } \\
\text { WC-96 } \\
\text { WC-112 } \\
\text { WC-123 } \\
\text { WC-132 } \\
\text { WC-145 }\end{array}$ & $\begin{array}{l}\text { ki Basin } \\
\text { ki Basin } \\
\text { ki Basin } \\
\text { ki Basin } \\
\text { ki Basin } \\
\text { ki Basin } \\
\text { ki Basin } \\
\text { ki Basin } \\
\text { ki Basin } \\
\text { ki Basin }\end{array}$ & $\begin{array}{l}11 / 28 / 90 \\
04 / 25 / 91 \\
07 / 29 / 91 \\
12 / 04 / 91 \\
04 / 20 / 92 \\
08 / 14 / 92 \\
12 / 04 / 92 \\
04 / 28 / 93 \\
08 / 04 / 93 \\
11 / 18 / 93\end{array}$ & $\begin{array}{c}\ldots \\
\ldots . . \\
\ldots \\
\ldots \\
\ldots . . \\
<0.001 \\
<0.001 \\
<0.001 \\
<0.001 \\
<0.0005 \\
<0.0005\end{array}$ & $\begin{array}{l}\quad . . \\
<0.1 \\
<0.1 \\
<0.1 \\
<0.1 \\
<0.1 \\
<0.1 \\
<0.1 \\
<0.1 \\
<0.1 \\
<0.05\end{array}$ & $\begin{array}{l}\quad \cdots \\
<0.05 \\
<0.05 \\
<0.05 \\
<0.05 \\
<0.05 \\
<0.05 \\
<0.05 \\
<0.05 \\
<0.05 \\
<0.0005\end{array}$ & $\begin{aligned} & \ldots . . \\
&<0.01 \\
&<0.01 \\
&<0.02 \\
&<0.01 \\
&<0.01 \\
&<0.01 \\
&<0.01 \\
&<0.01 \\
& 0.02 \\
&<0.01\end{aligned}$ & $\begin{array}{c}\ldots . . \\
\ldots \\
\ldots . \\
\ldots . \\
<0.001 \\
<0.001 \\
<0.001 \\
0.001 \\
0.0005 \\
0.0006\end{array}$ & $\begin{array}{c}\ldots . . \\
\ldots . . \\
-. . \\
-. . \\
<0.002 \\
<0.002 \\
<0.002 \\
<0.002 \\
<0.002 \\
<0.002\end{array}$ & $\begin{array}{c}\ldots \\
\ldots \\
\ldots \\
\ldots \\
\ldots . . \\
<0.002 \\
0.002 \\
<0.002 \\
<0.002 \\
0.002 \\
<0.002\end{array}$ & $\begin{array}{c}\ldots . . \\
\ldots . . \\
\ldots \\
\ldots . . \\
<0.002 \\
<0.002 \\
<0.002 \\
<0.002 \\
<0.002 \\
<0.002\end{array}$ & $\begin{array}{l}\cdots \\
\ldots- \\
\ldots \\
\ldots \\
\ldots . \\
0.020 \\
0.078 \\
0.002 \\
0.11 \\
0.017 \\
0.009\end{array}$ & $\begin{array}{r}<0.01 \\
<0.01 \\
<0.02 \\
<0.01 \\
0.09 \\
0.04 \\
<0.01 \\
0.19 \\
0.02 \\
<0.01\end{array}$ & $\begin{array}{c}<0.1 \\
<0.1 \\
\ldots \\
\ldots \\
\ldots \\
\ldots \\
\ldots \\
\ldots \\
<0.000\end{array}$ & $\begin{array}{c}\ldots \\
\ldots \\
\ldots . \\
\cdots \\
\cdots \\
<0.0 \\
<0.01 \\
<0.01 \\
<0.01 \\
<0.01\end{array}$ & $\begin{array}{r}\quad \ldots . \\
<0.01 \\
<0.01 \\
<0.01 \\
<0.01 \\
0.01 \\
<0.01 \\
<0.01 \\
<0.01 \\
<0.01 \\
<0.01\end{array}$ \\
\hline $\begin{array}{l}\text { WC-21 } \\
\text { WC-22 } \\
\text { WC-35 } \\
\text { WC-48 } \\
\text { WC-68 } \\
\text { WC-89 } \\
\text { WC-105 } \\
\text { WC-118 }\end{array}$ & $\begin{array}{l}\text { VC-2B, Sulphur Springs } \\
\text { VC-2B, Sulphur Springs } \\
\text { VC-2B, Sulphur Springs } \\
\text { VC-2B, Sulphur Springs } \\
\text { VC-2B, Sulphur Springs } \\
\text { VC-2B, Sulphur Springs } \\
\text { VC-2B, Sulphur Springs } \\
\text { VC-2B, Sulphur Springs }\end{array}$ & $\begin{array}{c}10 / 24 / 90 \\
11 / 26 / 90 \\
04 / 23 / 91 \\
07 / 29 / 91 \\
12 / 05 / 91 \\
05 / 01 / 92 \\
2 / 02 / 92 \\
04 / 21 / 93\end{array}$ & $\begin{array}{c}<0.001 \\
\ldots \\
\ldots \\
\ldots \\
\ldots \\
<0.001 \\
<0.001 \\
<0.001\end{array}$ & $\begin{array}{l}\quad . . \\
<0.1 \\
<0.1 \\
<0.1 \\
<0.1 \\
<0.1 \\
<0.1 \\
<0.1 \\
<0.1\end{array}$ & $\begin{array}{l}<0.05 \\
<0.05 \\
<0.05 \\
<0.05 \\
<0.05 \\
<0.05 \\
<0.05 \\
<0.05\end{array}$ & $\begin{array}{c}\quad \ldots \\
0.01 \\
<0.01 \\
<0.01 \\
<0.02 \\
0.01 \\
<0.01 \\
<0.01 \\
<0.01\end{array}$ & $\begin{array}{c}<0.001 \\
-. \\
-. \\
-. \\
<0.001 \\
<0.001 \\
<0.001\end{array}$ & $\begin{array}{c}<0.002 \\
\ldots \\
\ldots \\
\ldots \\
\ldots \\
<0.002 \\
<0.002 \\
<0.002\end{array}$ & $\begin{array}{c}0.002 \\
\ldots \\
\ldots \\
\ldots \\
\ldots \\
0.005 \\
<0.002 \\
<0.002\end{array}$ & $\begin{array}{c}0.002 \\
\ldots \\
\ldots \\
\ldots \\
\cdots \\
-. \\
<0.002 \\
0.004 \\
<0.002\end{array}$ & $\begin{array}{c}\ldots . \\
0.002 \\
\cdots \\
\cdots \\
\cdots \\
\cdots \\
0.003 \\
<0.002 \\
0.02\end{array}$ & $\begin{array}{r}<0.01 \\
<0.01 \\
<0.01 \\
<0.02 \\
<0.01 \\
<0.01 \\
0.03 \\
<0.01\end{array}$ & $\begin{array}{r}<0.1 \\
<0.1 \\
<0.1 \\
\ldots \\
\ldots \\
\ldots \\
\ldots\end{array}$ & $\begin{array}{c}<0.01 \\
\cdots \\
\cdots- \\
\cdots \\
\cdots \\
<0 . \\
<0.01 \\
<0.01\end{array}$ & $\begin{array}{l}<0.01 \\
<0.01 \\
<0.01 \\
<0.01 \\
<0.01 \\
<0.01 \\
<0.01 \\
<0.01\end{array}$ \\
\hline
\end{tabular}




\begin{tabular}{|c|c|c|c|c|c|c|c|c|c|c|c|c|c|c|c|c|}
\hline Sample \# & Description & Date & $\begin{array}{c}\mathrm{Mo} \\
\mathrm{mg} / \mathrm{kg}\end{array}$ & $\begin{array}{l}\mathrm{NH4} \\
\mathrm{mg} / \mathrm{kg}\end{array}$ & $\begin{array}{l}\mathrm{Ni} \\
\mathrm{mg} / \mathrm{kg}\end{array}$ & $\begin{array}{l}\mathrm{NO} 2 \\
\mathrm{mg} / \mathrm{kg}\end{array}$ & $\begin{array}{l}\mathrm{NO3} \\
\mathrm{mg} / \mathrm{kg}\end{array}$ & $\begin{array}{l}\mathrm{Pb} \\
\mathrm{mg} / \mathrm{kg}\end{array}$ & $\begin{array}{l}\mathrm{PO4} \\
\mathrm{mg} / \mathrm{kg}\end{array}$ & $\begin{array}{c}\mathrm{Rb} \\
\mathrm{mg} / \mathrm{kg}\end{array}$ & $\begin{array}{l}\mathrm{Sb} \\
\mathrm{mg} / \mathrm{kg}\end{array}$ & $\begin{array}{l}\mathrm{Se} \\
\mathrm{mg} / \mathrm{kg}\end{array}$ & $\begin{array}{l}\mathrm{Si} \\
\mathrm{mg} / \mathrm{kg}\end{array}$ & $\begin{array}{l}\mathrm{S} 203 \\
\mathrm{mg} / \mathrm{kg}\end{array}$ & $\underset{\mathrm{mg} / \mathrm{kg}}{\mathrm{U}}$ & $\begin{array}{l}\mathrm{Zn} \\
\mathrm{mg} / \mathrm{kg}\end{array}$ \\
\hline $\begin{array}{l}\text { WC-133 } \\
\text { WC-147 }\end{array}$ & $\begin{array}{l}\text { Espanola Ranger Station } \\
\text { Espanola Ranger Station }\end{array}$ & $\begin{array}{l}08 / 05 / 93 \\
11 / 29 / 93\end{array}$ & $\begin{array}{l}<0.002 \\
<0.002\end{array}$ & $\begin{array}{l}6.37 \\
0.38\end{array}$ & $\begin{array}{l}<0.002 \\
<0.002\end{array}$ & $\begin{array}{r}4.92 \\
<0.01\end{array}$ & $\begin{array}{l}2.83 \\
0.43\end{array}$ & $\begin{array}{l}0.002 \\
0.002\end{array}$ & $\begin{array}{r}1.23 \\
<0.02\end{array}$ & $\begin{array}{l}<0.002 \\
<0.002\end{array}$ & $<0.001$ & $<\overline{0.001}$ & $\begin{array}{l}<0.01 \\
0.07\end{array}$ & $\begin{array}{l}<0.01 \\
<0.01\end{array}$ & $\cdots$ & $\begin{array}{l}0.07 \\
0.02\end{array}$ \\
\hline $\begin{array}{l}\text { WC-11 } \\
\text { WC-23 } \\
\text { WC-36 } \\
\text { WC-49 } \\
\text { WC-94 } \\
\text { WC-124 } \\
\text { WC-131 } \\
\text { WC-143 }\end{array}$ & $\begin{array}{l}\text { Boundary Peak Area } \\
\text { Boundary Peak Area } \\
\text { Boundary Peak Area } \\
\text { Boundary Peak Area } \\
\text { Boundary Peak Area } \\
\text { Boundary Peak Area } \\
\text { Boundary Peak Area } \\
\text { Boundary Peak Area }\end{array}$ & $\begin{array}{l}09 / 04 / 90 \\
11 / 26 / 90 \\
04 / 23 / 91 \\
07 / 29 / 91 \\
08 / 10 / 92 \\
04 / 28 / 93 \\
08 / 03 / 93 \\
11 / 16 / 93\end{array}$ & $\begin{array}{c}\ldots \\
\ldots \\
\cdots \\
<0.002 \\
<0.002 \\
<0.002 \\
<0.002\end{array}$ & $\begin{array}{l}0.18 \\
0.15 \\
0.07 \\
0.46 \\
0.44 \\
0.34 \\
2.19 \\
0.28\end{array}$ & $\begin{array}{c}\cdots \\
\cdots \\
\cdots \\
<0.002 \\
<0.002 \\
0.002 \\
0.002\end{array}$ & $\begin{array}{r}<0.05 \\
<0.01 \\
<0.01 \\
1.83 \\
<0.02 \\
<0.02 \\
0.03 \\
<0.01\end{array}$ & $\begin{array}{l}1.14 \\
0.83 \\
0.40 \\
0.78 \\
1.02 \\
0.66 \\
2.47 \\
0.79\end{array}$ & $\begin{array}{c}\cdots \\
\cdots \\
\cdots \\
\cdots \\
<.002 \\
0.002 \\
0.002 \\
0.006\end{array}$ & $\begin{array}{l}<0.1 \\
<0.05 \\
<0.05 \\
0.13 \\
<0.05 \\
<0.02 \\
1.32 \\
<0.02\end{array}$ & $\begin{array}{c}\cdots \\
\cdots \\
\cdots \\
<0.002 \\
<0.002 \\
<0.002 \\
<0.002\end{array}$ & $\begin{array}{c}\quad \ldots \\
<0.10 \\
<0.10 \\
<0.10 \\
\cdots \\
\cdots \\
- \\
<0.001\end{array}$ & $\begin{array}{c}-. \\
<0.1 \\
<0.1 \\
-- \\
\cdots \\
\cdots \\
\cdots \\
<0.001\end{array}$ & $\begin{array}{c}<0.1 \\
<0.1 \\
<0.1 \\
<0.1 \\
0.13 \\
<0.01 \\
0.06\end{array}$ & $\begin{array}{c}\cdots \\
\cdots \\
\cdots \\
<0.02 \\
<0.01 \\
<0.01 \\
<0.01\end{array}$ & $\begin{array}{c}<0.1 \\
<0.1 \\
\ldots \\
\ldots \\
\ldots \\
\ldots \\
\ldots\end{array}$ & $\begin{array}{l}0 . \overline{0} \\
0.04 \\
0.01 \\
0.01 \\
0.06 \\
0.10 \\
0.02\end{array}$ \\
\hline $\begin{array}{l}\text { WC-12 } \\
\text { WC-24 } \\
\text { WC-39 } \\
\text { WC-50 } \\
\text { WC-76 } \\
\text { WC-85 } \\
\text { WC-92 } \\
\text { WC-106 } \\
\text { WC-119 } \\
\text { WC-135 } \\
\text { WC-144 }\end{array}$ & $\begin{array}{l}\text { West of Guaje Mtn. } \\
\text { West of Guaje Mtn. } \\
\text { West of Guaje Mtn. } \\
\text { West of Guaje Mtn. } \\
\text { West of Guaje Mtn. } \\
\text { West of Guaje Mtn. } \\
\text { West of Guaje Mtn. } \\
\text { West of Guaje Mtn. } \\
\text { West of Guaje Mtn. } \\
\text { West of Guaje Mtn. } \\
\text { West of Guaje Mtn. }\end{array}$ & $\begin{array}{l}09 / 04 / 90 \\
11 / 27 / 90 \\
04 / 23 / 91 \\
07 / 29 / 91 \\
12 / 10 / 91 \\
04 / 29 / 92 \\
08 / 10 / 92 \\
12 / 02 / 92 \\
04 / 23 / 93 \\
08 / 06 / 93 \\
11 / 17 / 93\end{array}$ & $\begin{array}{c}\ldots . \\
\ldots . \\
\cdots- \\
\cdots \\
<0.002 \\
<0.002 \\
<0.002 \\
<0.002 \\
<0.002 \\
<0.002\end{array}$ & $\begin{array}{l}0.14 \\
0.10 \\
0.16 \\
0.21 \\
0.14 \\
0.22 \\
2.53 \\
0.13 \\
0.26 \\
0.71 \\
0.30\end{array}$ & $\begin{array}{r}\ldots \\
\ldots \\
\ldots \\
\ldots \\
0.006 \\
<0.002 \\
0.020 \\
0.004 \\
<0.002 \\
0.005\end{array}$ & $\begin{array}{r}<0.05 \\
<0.01 \\
<0.01 \\
0.05 \\
<0.01 \\
<0.02 \\
<0.02 \\
<0.02 \\
<0.02 \\
<0.01 \\
<0.01\end{array}$ & $\begin{array}{l}0.83 \\
0.66 \\
0.83 \\
0.59 \\
1.13 \\
0.88 \\
0.18 \\
1.11 \\
0.70 \\
1.61 \\
0.74\end{array}$ & $\begin{array}{c}\ldots \\
\cdots-. \\
\cdots \\
\cdots \\
<0.002 \\
<0.002 \\
0.002 \\
0.002 \\
0.004 \\
0.006\end{array}$ & $\begin{array}{l}<0.1 \\
<0.05 \\
<0.05 \\
<0.02 \\
<0.02 \\
<0.05 \\
0.28 \\
<0.05 \\
<0.02 \\
0.08 \\
<0.02\end{array}$ & $\begin{array}{c}\ldots \\
\ldots . . \\
\cdots \\
\cdots \\
<0.002 \\
<0.002 \\
<0.002 \\
<0.002 \\
<0.002 \\
<0.002\end{array}$ & 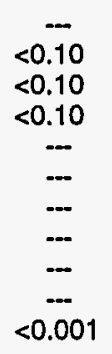 & 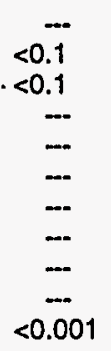 & $\begin{array}{c}<-. . \\
<0.1 \\
<0.1 \\
<0.1 \\
<0.1 \\
<0.1 \\
<0.1 \\
0.09 \\
0.11 \\
<0.01 \\
0.08\end{array}$ & $\begin{array}{c}\ldots \\
\ldots \\
\ldots \\
\ldots \\
\ldots \\
<0.02 \\
<0.01 \\
<0.01 \\
<0.01 \\
<0.01\end{array}$ & $\begin{array}{c}-\overline{0} \\
<0.1 \\
<0.1 \\
-. \\
-. \\
- \\
- \\
- \\
- \\
- \\
-\end{array}$ & $\begin{array}{r}0 .-03 \\
0.04 \\
0.01 \\
0.03 \\
0.05 \\
0.03 \\
0.06 \\
<0.01 \\
0.11 \\
0.03\end{array}$ \\
\hline $\begin{array}{l}\text { WC-13 } \\
\text { WC-29 } \\
\text { WC-47 } \\
\text { WC-52 } \\
\text { WC-66 } \\
\text { WC-81 } \\
\text { WC-96 } \\
\text { WC-112 } \\
\text { WC-123 } \\
\text { WC-132 } \\
\text { WC-145 }\end{array}$ & $\begin{array}{l}\text { Santa Fe Ski Basin } \\
\text { Santa Fe Ski Basin } \\
\text { Santa Fe Ski Basin } \\
\text { Santa Fe Ski Basin } \\
\text { Santa Fe Ski Basin } \\
\text { Santa Fe Ski Basin } \\
\text { Santa Fe Ski Basin } \\
\text { Santa Fe Ski Basin } \\
\text { Santa Fe Ski Basin } \\
\text { Santa Fe Ski Basin } \\
\text { Santa Fe Ski Basin }\end{array}$ & $\begin{array}{l}09 / 05 / 90 \\
11 / 28 / 90 \\
04 / 25 / 91 \\
07 / 29 / 91 \\
12 / 04 / 91 \\
04 / 20 / 92 \\
08 / 14 / 92 \\
12 / 04 / 92 \\
04 / 28 / 93 \\
08 / 04 / 93 \\
11 / 18 / 93\end{array}$ & $\begin{array}{c}\cdots \\
\cdots \\
\cdots \\
\cdots \\
<0 . \\
<0.002 \\
<0.002 \\
<0.002 \\
<0.002 \\
<0.002\end{array}$ & $\begin{array}{l}0.28 \\
0.26 \\
0.11 \\
0.15 \\
0.06 \\
0.35 \\
0.29 \\
0.23 \\
0.39 \\
0.51 \\
0.22\end{array}$ & $\begin{array}{c}\ldots \\
\ldots \\
\cdots \\
\cdots \\
0 . \\
0.033 \\
<0.002 \\
<0.002 \\
<0.002 \\
<0.002 \\
0.002\end{array}$ & $\begin{array}{l}<0.05 \\
<0.01 \\
<0.01 \\
0.03 \\
0.25 \\
<0.02 \\
<0.02 \\
<0.02 \\
<0.02 \\
<0.01 \\
<0.01\end{array}$ & $\begin{array}{l}0.88 \\
0.68 \\
0.83 \\
0.49 \\
0.11 \\
0.88 \\
1.06 \\
0.13 \\
1.22 \\
1.48 \\
1.04\end{array}$ & $\begin{array}{c}\bar{y} \\
\overline{-} \\
\overline{-} \\
\overline{-} \\
<0.002 \\
<0.002 \\
0.002 \\
0.008 \\
0.003 \\
0.004\end{array}$ & $\begin{array}{l}<0.1 \\
<0.05 \\
<0.05 \\
<0.02 \\
<0.02 \\
<0.05 \\
<0.05 \\
<0.05 \\
<0.02 \\
0.04 \\
<0.02\end{array}$ & $\begin{array}{c}\cdots \\
\cdots- \\
\cdots- \\
\cdots \\
<0 . \\
<0.002 \\
<0.002 \\
<0.002 \\
<0.002 \\
<0.002 \\
<0.002\end{array}$ & $\begin{array}{l}<\overline{10} \\
<0.10 \\
<0.10 \\
\overline{-} \\
\overline{-} \\
\overline{-} \\
\overline{-}\end{array}$ & $\begin{array}{c}-\overline{-} \\
<0.1 \\
\overline{-} \\
\overline{-} \\
\overline{-} \\
\overline{-} \\
\overline{-} \\
<0.001\end{array}$ & $\begin{aligned} &<-. \\
&<0.1 \\
&<0.1 \\
&<0.1 \\
&<0.1 \\
&<0.1 \\
&<0.1 \\
& 0.08 \\
& 0.25 \\
&<0.01 \\
& 0.04\end{aligned}$ & $\begin{array}{l}\overline{-} \\
\overline{-} \\
\overline{-} \\
\overline{-} \\
<\overline{-} \\
<0.02 \\
<0.01 \\
<0.01 \\
<0.01\end{array}$ & $\begin{array}{l}-. \\
<0.1 \\
<0.1 \\
-- \\
-- \\
-- \\
- \\
- \\
- \\
-\end{array}$ & $\begin{array}{l}-.-2 \\
0.21 \\
0.05 \\
0.08 \\
0.04 \\
0.09 \\
0.36 \\
0.05 \\
0.29 \\
0.30 \\
0.10\end{array}$ \\
\hline $\begin{array}{l}\text { WC-16 } \\
\text { WC-21 } \\
\text { WC-22 } \\
\text { WC-35 } \\
\text { WC-48 } \\
\text { WC-68 } \\
\text { WC-89 } \\
\text { WC-105 } \\
\text { WC-118 }\end{array}$ & $\begin{array}{l}\text { VC-2B, Sulphur Springs } \\
\text { VC-2B, Sulphur Springs } \\
\text { VC-2B, Sulphur Springs } \\
\text { VC-2B, Sulphur Springs } \\
\text { VC-2B, Sulphur Springs } \\
\text { VC-2B, Sulphur Springs } \\
\text { VC-2B, Sulphur Springs } \\
\text { VC-2B, Sulphur Springs } \\
\text { VC-2B, Sulphur Springs }\end{array}$ & $\begin{array}{l}09 / 06 / 90 \\
10 / 24 / 90 \\
11 / 26 / 90 \\
04 / 23 / 91 \\
07 / 29 / 91 \\
12 / 05 / 91 \\
05 / 01 / 92 \\
12 / 02 / 92 \\
04 / 21 / 93 \\
\end{array}$ & $\begin{array}{c}<0.002 \\
\ldots \\
\ldots \\
\ldots \\
<0.002 \\
<0.002 \\
<0.002\end{array}$ & $\begin{array}{l}0.56 \\
1.43 \\
0.36 \\
0.15 \\
2.70 \\
1.54 \\
0.54 \\
0.14 \\
0.18\end{array}$ & $\begin{array}{c}\overline{0.002} \\
\overline{-} \\
\overline{-} \\
\overline{0} \\
0.006 \\
<0.002 \\
<0.002\end{array}$ & $\begin{array}{l}<0.05 \\
4.5 \\
<0.01 \\
<0.01 \\
13.0 \\
<0.01 \\
<0.02 \\
<0.02 \\
0.02\end{array}$ & $\begin{array}{l}1.00 \\
1.13 \\
0.57 \\
0.85 \\
0.97 \\
0.52 \\
0.66 \\
0.15 \\
0.50\end{array}$ & $\begin{array}{c}<0.002 \\
\overline{-} \\
\overline{-} \\
\overline{-} \\
<0.002 \\
<0.002 \\
0.002\end{array}$ & $\begin{array}{l}<0.1 \\
0.45 \\
<0.05 \\
<0.05 \\
0.93 \\
0.34 \\
<0.05 \\
<0.05 \\
<0.02\end{array}$ & $\begin{array}{c}<0.002 \\
\cdots \\
\cdots \\
\cdots \\
\cdots \\
<0.002 \\
<0.002 \\
<0.002\end{array}$ & $\begin{array}{l}<0 . \\
<0.10 \\
<0.10 \\
<0.10 \\
<0.10 \\
\ldots \\
\overline{-} \\
- \\
-\end{array}$ & $\begin{array}{r}<0.10 \\
<0.10 \\
<0.10 \\
\overline{-} \\
\overline{-} \\
\overline{-}\end{array}$ & $\begin{array}{c}<0.1 \\
<0.1 \\
<0.1 \\
0.8 \\
<0.1 \\
<0.1 \\
0.14 \\
0.50\end{array}$ & $\begin{array}{c}<\overline{0.01} \\
\ldots \\
\ldots \\
\ldots \\
\overline{-} \\
<0.01 \\
<0.01\end{array}$ & $\begin{array}{l}<\overline{0.1} \\
<0.1 \\
<0.1 \\
-- \\
- \\
- \\
-\end{array}$ & $\begin{array}{r}0.02 \\
0.09 \\
0.14 \\
0.03 \\
0.01 \\
0.05 \\
0.02 \\
<0.01 \\
\end{array}$ \\
\hline
\end{tabular}




\begin{tabular}{|c|c|c|c|c|c|c|c|c|c|c|c|c|c|c|c|}
\hline $\begin{array}{l}\text { Sample \# } \\
\text { WC-130a } \\
\text { WC-142 }\end{array}$ & \begin{tabular}{l}
\multicolumn{1}{c}{ Description } \\
VC-2B, Sulphur Springs \\
VC-2B, Sulphur Springs
\end{tabular} & $\begin{array}{c}\text { Date } \\
08 / 03 / 93 \\
11 / 16 / 93\end{array}$ & $\begin{array}{c}\mathrm{Ag} \\
\mathrm{mg} / \mathrm{kg} \\
<0.0005 \\
<0.0005\end{array}$ & $\begin{array}{c}\begin{array}{c}\mathrm{Al} \\
\mathrm{mg} / \mathrm{kg}\end{array} \\
<0.1 \\
<0.05\end{array}$ & $\begin{array}{c}\text { As } \\
\mathrm{mg} / \mathrm{kg} \\
<0.05 \\
0.0005\end{array}$ & $\begin{array}{c}\mathrm{Ba} \\
\mathrm{mg} / \mathrm{kg}\end{array}$ & $\begin{array}{c}\mathrm{Cd} \\
\mathrm{mg} / \mathrm{kg}\end{array}$ & $\begin{array}{c}\mathrm{Co} \\
\mathrm{mg} / \mathrm{kg}\end{array}$ & $\begin{array}{c}\begin{array}{c}\mathrm{Cr} \\
\mathrm{mg} / \mathrm{kg}\end{array} \\
0.002 \\
<0.002\end{array}$ & $\begin{array}{c}\begin{array}{c}\mathrm{Cs} \\
\mathrm{mg} / \mathrm{kg}\end{array} \\
<0.002 \\
<0.002\end{array}$ & $\begin{array}{c}\begin{array}{c}\mathrm{Cu} \\
\mathrm{mg} / \mathrm{kg}\end{array} \\
0.008 \\
0.003\end{array}$ & $\begin{array}{c}\begin{array}{c}\mathrm{Fe} \\
\mathrm{mg} / \mathrm{kg}\end{array} \\
0.07 \\
0.01\end{array}$ & $\begin{array}{c}\mathrm{Hg} \\
\mathrm{mg} / \mathrm{kg}\end{array}$ & $\begin{array}{c}\frac{1}{\mathrm{mg} / \mathrm{kg}} \\
<0.01 \\
<0.01\end{array}$ & $\begin{array}{c}\begin{array}{c}\mathrm{Mn} \\
\mathrm{mg} / \mathrm{kg}\end{array} \\
<0.01 \\
<0.01\end{array}$ \\
\hline $\begin{array}{l}\text { WC-17 } \\
\text { WC-26 } \\
\text { WC-38 } \\
\text { WC-53 } \\
\text { WC-73 } \\
\text { WC-82 } \\
\text { WC-95 } \\
\text { WC-111 } \\
\text { WC-121 } \\
\text { WC-150 }\end{array}$ & $\begin{array}{l}\text { Tech Area } 49 \\
\text { Tech Area } 49 \\
\text { Tech Area } 49 \\
\text { Tech Area } 49 \\
\text { Tech Area } 49 \\
\text { Tech Area } 49 \\
\text { Tech Area } 49 \\
\text { Tech Area } 49 \\
\text { Tech Area } 49 \\
\text { Tech Area } 49\end{array}$ & $\begin{array}{c}09 / 06 / 90 \\
11 / 27 / 90 \\
04 / 23 / 91 \\
08 / 01 / 91 \\
2 / 16 / 91 \\
04 / 28 / 92 \\
08 / 10 / 92 \\
2 / 03 / 92 \\
04 / 23 / 93 \\
11 / 29 / 93\end{array}$ & $\begin{array}{c}\ldots . \\
\cdots-. \\
\ldots . \\
\ldots . \\
<0.001 \\
<0.001 \\
<0.001 \\
<0.001 \\
<0.0005\end{array}$ & $\begin{array}{l}<0.1 \\
<0.1 \\
<0.1 \\
<0.1 \\
<0.1 \\
<0.1 \\
<0.1 \\
<0.1 \\
<0.05\end{array}$ & $\begin{array}{l}=\overline{0} \\
=0.05 \\
=0.05 \\
0.05 \\
0.05 \\
=0.05 \\
0.05 \\
=0.05 \\
0.0005\end{array}$ & $\begin{array}{l}<0.01 \\
<0.01 \\
<0.02 \\
<0.01 \\
<0.01 \\
<0.01 \\
<0.01 \\
<0.01 \\
<0.01\end{array}$ & $\begin{array}{c}\ldots \\
\ldots \\
\ldots \\
\ldots \\
0.001 \\
<0.001 \\
<0.001 \\
0.003 \\
0.0007\end{array}$ & $\begin{array}{c}\ldots . \\
\ldots . . \\
\ldots . . \\
\ldots . \\
<0.002 \\
<0.002 \\
<0.002 \\
<0.002 \\
<0.002\end{array}$ & $\begin{array}{c}\ldots . \\
\ldots . \\
\ldots \\
\ldots \\
<0 . \\
<0.002 \\
<0.002 \\
<0.002 \\
<0.002 \\
<0.002\end{array}$ & $\begin{array}{c}\ldots . \\
\ldots \\
\ldots . \\
\ldots \\
\ldots . \\
<0.002 \\
<0.002 \\
0.003 \\
0.005 \\
<0.002\end{array}$ & $\begin{array}{c}\ldots \\
\ldots . \\
\ldots \\
\ldots . \\
\ldots . . \\
0.014 \\
<0.002 \\
0.005 \\
0.006 \\
0.010\end{array}$ & $\begin{array}{r}<0.01 \\
<0.01 \\
<0.02 \\
0.03 \\
<0.01 \\
0.02 \\
<0.01 \\
<0.01 \\
0.01\end{array}$ & $\begin{array}{c}<0.1 \\
<0.1 \\
\ldots . \\
\cdots \\
\cdots- \\
\cdots \\
\cdots \\
<0.000\end{array}$ & $\begin{array}{l}\ldots \\
- \\
- \\
-. \\
- \\
-- \\
<0.02 \\
<0.01 \\
<0.01 \\
<0.01\end{array}$ & $\begin{array}{l}<0.01 \\
<0.01 \\
<0.01 \\
<0.01 \\
<0.01 \\
<0.01 \\
<0.01 \\
<0.01 \\
<0.01\end{array}$ \\
\hline $\begin{array}{l}\text { WC-27 } \\
\text { WC-40 } \\
\text { WC-59 } \\
\text { WC-75 } \\
\text { WC-88 } \\
\text { WC-100 } \\
\text { WC-109 } \\
\text { WC-122 } \\
\text { WC-139 } \\
\text { WC-151 }\end{array}$ & $\begin{array}{l}\text { Tech Area } 51 \\
\text { Tech Area } 51 \\
\text { Tech Area } 51 \\
\text { Tech Area } 51 \\
\text { Tech Area } 51 \\
\text { Tech Area } 51 \\
\text { Tech Area } 51 \\
\text { Tech Area } 51 \\
\text { Tech Area } 51 \\
\text { Tech Area } 51\end{array}$ & $\begin{array}{l}11 / 27 / 90 \\
04 / 24 / 91 \\
08 / 05 / 91 \\
12 / 16 / 91 \\
04 / 29 / 92 \\
08 / 14 / 92 \\
12 / 03 / 92 \\
04 / 23 / 93 \\
08 / 13 / 93 \\
11 / 29 / 93\end{array}$ & $\begin{array}{c}\ldots \\
\ldots \\
\cdots \\
\cdots \\
\cdots \\
<0.001 \\
<0.001 \\
<0.001 \\
<0.001 \\
<0.0005 \\
<0.0005\end{array}$ & $\begin{array}{l}<0.1 \\
<0.1 \\
<0.1 \\
<0.1 \\
<0.1 \\
<0.1 \\
<0.1 \\
<0.1 \\
<0.1 \\
0.17\end{array}$ & $\begin{array}{l}<0.05 \\
<0.05 \\
<0.05 \\
<0.05 \\
<0.05 \\
<0.05 \\
<0.05 \\
<0.05 \\
<0.05 \\
0\end{array}$ & $\begin{array}{l}<0.01 \\
<0.01 \\
<0.02 \\
<0.01 \\
<0.01 \\
<0.01 \\
<0.01 \\
<0.01 \\
<0.01 \\
0.01\end{array}$ & $\begin{array}{c}\cdots \\
\cdots \\
\cdots \\
\cdots \\
0.001 \\
0.002 \\
<0.001 \\
<0.001 \\
0.0003 \\
0.0006\end{array}$ & $\begin{array}{c}\ldots . \\
\ldots \\
\ldots . . \\
<0.002 \\
<0.002 \\
<0.002 \\
<0.002 \\
<0.002 \\
<0.002\end{array}$ & $\begin{array}{c}\ldots \\
\cdots \\
\cdots \\
\cdots \\
\ldots . . \\
0.003 \\
<0.002 \\
<0.002 \\
<0.002 \\
<0.002 \\
<0.002\end{array}$ & $\begin{array}{c}\ldots \\
\ldots \\
\ldots \\
\cdots \\
\cdots \\
<0.002 \\
0.003 \\
0.003 \\
<0.002 \\
<0.002 \\
<0.002\end{array}$ & $\begin{array}{c}\cdots \\
\cdots \\
\cdots \\
\cdots \\
\cdots \\
0.003 \\
0.011 \\
0.002 \\
0.010 \\
0.005 \\
0.007\end{array}$ & $\begin{array}{r}<0.01 \\
<0.01 \\
0.02 \\
<0.01 \\
<0.01 \\
<0.01 \\
<0.01 \\
<0.01 \\
<0.01 \\
0.01\end{array}$ & $\begin{array}{c}<0.1 \\
<0.1 \\
\ldots \\
\ldots . \\
\ldots \\
\cdots \\
\ldots \\
\cdots \\
<0.0005\end{array}$ & $\begin{array}{c}\ldots \\
\ldots \\
\ldots \\
\ldots \\
\ldots \\
- \\
<0.02 \\
<0.01 \\
<0.01 \\
<0.01 \\
<0.01\end{array}$ & $\begin{array}{r}<0.01 \\
<0.01 \\
<0.01 \\
0.01 \\
<0.01 \\
<0.01 \\
<0.01 \\
<0.01 \\
<0.01 \\
<0.01\end{array}$ \\
\hline $\begin{array}{l}\text { WC-60 } \\
\text { WC-74 } \\
\text { WC-91 } \\
\text { WC-104 } \\
\text { WC-117 } \\
\text { WC-120 } \\
\text { WC-141 } \\
\text { WC-154 }\end{array}$ & $\begin{array}{l}\text { Kim Manley's House } \\
\text { Kim Manley's House } \\
\text { Kim Manley's House } \\
\text { Kim Manley's House } \\
\text { Kim Manley's House } \\
\text { Kim Manley's House } \\
\text { Kim Manley's House }\end{array}$ & $\begin{array}{l}08 / 05 / 91 \\
12 / 15 / 91 \\
05 / 27 / 92 \\
08 / 20 / 92 \\
12 / 18 / 92 \\
04 / 23 / 93 \\
08 / 13 / 93 \\
11 / 29 / 93\end{array}$ & $\begin{array}{c}\ldots . \\
<0.001 \\
<0.001 \\
<0.001 \\
<0.001 \\
<0.0005 \\
<0.0005\end{array}$ & $\begin{array}{l}<0.1 \\
<0.1 \\
<0.1 \\
<0.1 \\
<0.1 \\
<0.1 \\
<0.1 \\
<0.05\end{array}$ & $\begin{array}{l}<0.05 \\
<0.05 \\
<0.05 \\
<0.05 \\
<0.05 \\
<0.05 \\
<0.05 \\
0.0012\end{array}$ & $\begin{array}{l}<0.01 \\
<0.01 \\
<0.01 \\
<0.01 \\
<0.01 \\
<0.01 \\
<0.01\end{array}$ & $\begin{array}{c}\ldots . \\
\ldots .001 \\
<0.001 \\
<0.001 \\
<0.001 \\
0.0002 \\
<0.0005\end{array}$ & $\begin{array}{c}\ldots . . \\
<0.002 \\
<0.002 \\
<0.002 \\
<0.002 \\
<0.002 \\
<0.002\end{array}$ & $\begin{array}{c}\ldots \\
\ldots 0.002 \\
<0.002 \\
<0.002 \\
<0.002 \\
<0.002 \\
<0.002\end{array}$ & $\begin{array}{c}\ldots . . \\
<0.002 \\
<0.002 \\
<0.002 \\
<0.002 \\
<0.002 \\
<0.002\end{array}$ & $\begin{array}{c}\ldots . \\
\ldots . . \\
0.008 \\
0.006 \\
0.004 \\
0.011 \\
0.009 \\
0.015\end{array}$ & $\begin{array}{r}<0.02 \\
0.02 \\
<0.01 \\
0.08 \\
<0.01 \\
<0.01 \\
0.04 \\
<0.01\end{array}$ & $\begin{array}{c}\ldots . \\
\ldots \\
\ldots- \\
\ldots \\
\ldots \\
\cdots \\
-. \\
<0.005\end{array}$ & $\begin{array}{l}-- \\
\overline{-} \\
<0.02 \\
<0.01 \\
<0.01 \\
<0.01 \\
<0.01\end{array}$ & $\begin{array}{l}<0.01 \\
<0.01 \\
<0.01 \\
<0.01 \\
<0.01 \\
<0.01 \\
<0.01 \\
<0.01\end{array}$ \\
\hline
\end{tabular}




\begin{tabular}{|c|c|c|c|c|c|c|c|c|c|c|c|c|c|c|c|c|}
\hline Sample \# & Description & Date & $\begin{array}{c}\mathrm{Mo} \\
\mathrm{mg} / \mathrm{kg}\end{array}$ & $\begin{array}{l}\mathrm{NH4} \\
\mathrm{mg} / \mathrm{kg}\end{array}$ & $\begin{array}{c}\mathrm{Ni} \\
\mathrm{mg} / \mathrm{kg}\end{array}$ & $\begin{array}{l}\mathrm{NO} 2 \\
\mathrm{mg} / \mathrm{kg}\end{array}$ & $\begin{array}{c}\mathrm{NO3} \\
\mathrm{mg} / \mathrm{kg}\end{array}$ & $\begin{array}{c}\mathrm{Pb} \\
\mathrm{mg} / \mathrm{kg}\end{array}$ & $\begin{array}{l}\mathrm{PO4} \\
\mathrm{mg} / \mathrm{kg}\end{array}$ & $\begin{array}{c}\mathrm{Rb} \\
\mathrm{mg} / \mathrm{kg}\end{array}$ & $\begin{array}{c}\mathrm{Sb} \\
\mathrm{mg} / \mathrm{kg}\end{array}$ & $\begin{array}{c}\mathrm{Se} \\
\mathrm{mg} / \mathrm{kg}\end{array}$ & $\begin{array}{c}\mathrm{Si} \\
\mathrm{mg} / \mathrm{kg}\end{array}$ & $\begin{array}{l}\mathrm{S} 2 \mathrm{O} 3 \\
\mathrm{mg} / \mathrm{kg}\end{array}$ & $\begin{array}{c}\mathrm{U} \\
\mathrm{mg} / \mathrm{kg}\end{array}$ & $\begin{array}{c}\mathrm{Zn} \\
\mathrm{mg} / \mathrm{kg}\end{array}$ \\
\hline $\begin{array}{l}\text { WC-130a } \\
\text { WC-142 }\end{array}$ & $\begin{array}{l}\text { VC-2B, Sulphur Springs } \\
\text { VC-2B, Sulphur Springs }\end{array}$ & $\begin{array}{l}08 / 03 / 93 \\
11 / 16 / 93\end{array}$ & $\begin{array}{l}<0.002 \\
<0.002\end{array}$ & $\begin{array}{l}1.66 \\
1.00\end{array}$ & $\begin{array}{l}0.002 \\
0.002\end{array}$ & $\begin{array}{r}0.02 \\
<0.01\end{array}$ & $\begin{array}{l}2.03 \\
0.63\end{array}$ & $\begin{array}{l}0.004 \\
0.006\end{array}$ & $\begin{array}{r}0.54 \\
<0.02\end{array}$ & $\begin{array}{c}<0.002 \\
0\end{array}$ & $<\overline{0.001}$ & $<0 . \overline{001}$ & $\begin{array}{r}<0.01 \\
0.07\end{array}$ & $\begin{array}{l}<0.01 \\
<0.01\end{array}$ & - & $\begin{array}{l}0.11 \\
0.03\end{array}$ \\
\hline $\begin{array}{l}\text { WC-17 } \\
\text { WC-26 } \\
\text { WC-38 } \\
\text { WC-53 } \\
\text { WC-73 } \\
\text { WC-82 } \\
\text { WC-95 } \\
\text { WC-111 } \\
\text { WC-121 } \\
\text { WC-150 }\end{array}$ & $\begin{array}{l}\text { Tech Area } 49 \\
\text { Tech Area } 49 \\
\text { Tech Area } 49 \\
\text { Tech Area } 49 \\
\text { Tech Area } 49 \\
\text { Tech Area } 49 \\
\text { Tech Area } 49 \\
\text { Tech Area } 49 \\
\text { Tech Area } 49 \\
\text { Tech Area } 49\end{array}$ & $\begin{array}{l}09 / 06 / 90 \\
11 / 27 / 90 \\
04 / 23 / 91 \\
08 / 01 / 91 \\
12 / 16 / 91 \\
04 / 28 / 92 \\
08 / 10 / 92 \\
12 / 03 / 92 \\
04 / 23 / 93 \\
11 / 29 / 93\end{array}$ & $\begin{array}{c}\ldots \\
\ldots .- \\
\cdots- \\
\cdots . . \\
<0.002 \\
<0.002 \\
<0.002 \\
<0.002 \\
<0.002\end{array}$ & $\begin{array}{r}2.01 \\
0.76 \\
0.59 \\
1.28 \\
0.76 \\
0.88 \\
5.49 \\
10.00 \\
0.50 \\
12.50\end{array}$ & $\begin{array}{c}\ldots \\
\ldots \\
\ldots \\
\ldots \\
0 . \\
0.006 \\
<0.002 \\
<0.002 \\
<0.002 \\
0.005\end{array}$ & $\begin{array}{r}<0.05 \\
0.02 \\
0.02 \\
0.19 \\
0.03 \\
<0.02 \\
0.13 \\
<0.02 \\
<0.02 \\
10.60\end{array}$ & $\begin{array}{l}1.87 \\
1.06 \\
1.67 \\
0.75 \\
1.10 \\
1.75 \\
1.61 \\
1.29 \\
1.25 \\
1.47\end{array}$ & $\begin{array}{r}\ldots . . \\
\ldots . \\
\ldots . \\
\ldots . . \\
0.002 \\
<0.002 \\
0.002 \\
0.005 \\
0.006\end{array}$ & $\begin{array}{l}0.77 \\
0.09 \\
0.30 \\
0.37 \\
0.15 \\
<0.05 \\
1.80 \\
2.15 \\
0.02 \\
2.13\end{array}$ & $\begin{array}{c}\ldots \\
\ldots . . \\
\ldots . \\
\ldots \\
<0.002 \\
<0.002 \\
<0.002 \\
<0.002 \\
<0.002\end{array}$ & $\begin{array}{c}<-. \\
<0.1 \\
<0.1 \\
<0.1 \\
-. \\
\cdots \\
\cdots \\
- \\
<0.001\end{array}$ & $\begin{array}{c}\ddot{0.1} \\
<0.1 \\
\cdots \\
\cdots \\
\cdots \\
\cdots \\
\cdots \\
-.001\end{array}$ & $\begin{array}{l}<0.1 \\
<0.1 \\
<0.1 \\
<0.1 \\
<0.1 \\
<0.1 \\
0.11 \\
0.02 \\
0.10\end{array}$ & $\begin{array}{c}\ldots \\
\ldots . . \\
\ldots- \\
\ldots . \\
\ldots . \\
\cdots \\
<0.02 \\
<0.01 \\
<0.01 \\
<0.01\end{array}$ & $\begin{array}{c}\ldots . . \\
<0.1 \\
<0.1 \\
\ldots- \\
\ldots \\
\ldots \\
\ldots \\
\ldots \\
\ldots \\
\ldots \\
. .-\end{array}$ & $\begin{array}{l}-0.35 \\
0.53 \\
0.15 \\
0.22 \\
0.82 \\
0.23 \\
0.16 \\
0.63 \\
0.24\end{array}$ \\
\hline $\begin{array}{l}\text { WC-18 } \\
\text { WC-27 } \\
\text { WC-40 } \\
\text { WC-59 } \\
\text { WC-75 } \\
\text { WC-88 } \\
\text { WC-100 } \\
\text { WC-109 } \\
\text { WC-122 } \\
\text { WC-139 } \\
\text { WC-151 }\end{array}$ & $\begin{array}{l}\text { Tech Area } 51 \\
\text { Tech Area } 51 \\
\text { Tech Area } 51 \\
\text { Tech Area } 51 \\
\text { Tech Area } 51 \\
\text { Tech Area } 51 \\
\text { Tech Area } 51 \\
\text { Tech Area } 51 \\
\text { Tech Area } 51 \\
\text { Tech Area } 51 \\
\text { Tech Area } 51\end{array}$ & $\begin{array}{l}09 / 06 / 90 \\
11 / 27 / 90 \\
04 / 24 / 91 \\
08 / 05 / 91 \\
12 / 16 / 91 \\
04 / 29 / 92 \\
08 / 14 / 92 \\
12 / 03 / 92 \\
04 / 23 / 93 \\
08 / 13 / 93 \\
11 / 29 / 93\end{array}$ & $\begin{array}{c}\ldots . . \\
\cdots \\
\cdots . \\
\cdots \\
<0.0 \\
<0.002 \\
<0.002 \\
<0.002 \\
<0.002 \\
<0.002\end{array}$ & $\begin{array}{l}0.32 \\
0.36 \\
0.77 \\
0.24 \\
0.18 \\
0.42 \\
0.52 \\
0.40 \\
0.29 \\
1.58 \\
0.32\end{array}$ & $\begin{array}{c}\ldots \\
\ldots \\
\ldots \\
\ldots \\
0.005 \\
0.002 \\
<0.002 \\
<0.002 \\
<0.002 \\
0.005\end{array}$ & $\begin{array}{r}<0.05 \\
0.01 \\
0.02 \\
0.03 \\
<0.01 \\
<0.02 \\
<0.02 \\
<0.02 \\
<0.02 \\
<0.01 \\
0.02\end{array}$ & $\begin{array}{l}1.07 \\
0.71 \\
0.95 \\
0.58 \\
0.73 \\
1.17 \\
1.24 \\
1.35 \\
0.73 \\
3.25 \\
0.92\end{array}$ & $\begin{array}{c}\cdots \\
\cdots-. \\
\cdots \\
\cdots \\
\cdots . \\
0.002 \\
0.002 \\
<0.002 \\
0.002 \\
0.002 \\
0.004\end{array}$ & $\begin{array}{l}<0.1 \\
<0.05 \\
0.12 \\
<0.02 \\
<0.02 \\
<0.05 \\
<0.05 \\
<0.05 \\
<0.02 \\
0.22 \\
<0.02\end{array}$ & $\begin{array}{c}\ldots \\
\ldots \\
\ldots . \\
\cdots \\
\cdots \\
<0.002 \\
<0.002 \\
<0.002 \\
<0.002 \\
<0.002 \\
<0.002\end{array}$ & $\begin{array}{c}<-. \\
<0.1 \\
<0.1 \\
<0.1 \\
-\overline{-.} \\
\cdots \\
\cdots \\
\overline{-} \\
<0.001\end{array}$ & 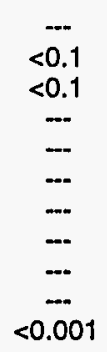 & $\begin{array}{l}<0.1 \\
<0.1 \\
<0.1 \\
<0.1 \\
<0.1 \\
<0.1 \\
0.13 \\
<0.01 \\
<0.01 \\
<0.05\end{array}$ & $\begin{array}{l}\ldots \\
\ldots \\
\ldots \\
\ldots \\
\ldots \\
\ldots 0 . \\
<02 \\
<0.01 \\
<0.01 \\
<0.01 \\
<0.01\end{array}$ & $\begin{array}{c}\ldots . . \\
<0.1 \\
<0.1 \\
-- \\
\cdots \\
\cdots \\
\cdots- \\
\cdots \\
\cdots \\
\cdots\end{array}$ & $\begin{array}{l}\ldots . . \\
0.08 \\
0.03 \\
0.04 \\
0.05 \\
0.05 \\
0.04 \\
0.05 \\
0.02 \\
0.11 \\
0.03\end{array}$ \\
\hline $\begin{array}{l}\text { WC-60 } \\
\text { WC-74 } \\
\text { WC-91 } \\
\text { WC-104 } \\
\text { WC-117 } \\
\text { WC-120 } \\
\text { WC-141 } \\
\text { WC-154 }\end{array}$ & $\begin{array}{l}\text { Kim Manley's House } \\
\text { Kim Manley's House } \\
\text { Kim Manley's House } \\
\text { Kim Manley's House } \\
\text { Kim Manley's House } \\
\text { Kim Manley's House } \\
\text { Kim Manley's House } \\
\text { Kim Manley's House }\end{array}$ & $\begin{array}{l}08 / 05 / 91 \\
12 / 15 / 91 \\
05 / 27 / 92 \\
08 / 20 / 92 \\
12 / 18 / 92 \\
04 / 23 / 93 \\
08 / 13 / 93 \\
11 / 29 / 93\end{array}$ & $\begin{array}{c}\cdots \\
<0.002 \\
<0.002 \\
<0.002 \\
<0.002 \\
<0.002 \\
<0.002\end{array}$ & $\begin{array}{l}0.12 \\
0.13 \\
0.35 \\
0.50 \\
0.16 \\
0.24 \\
1.03 \\
0.30 \\
\end{array}$ & $\begin{array}{c}\ldots \\
0 . \overline{-} \\
<0.002 \\
0.003 \\
<0.002 \\
<0.002 \\
0.004 \\
\end{array}$ & $\begin{array}{r}0.04 \\
<0.01 \\
<0.02 \\
<0.02 \\
<0.02 \\
<0.02 \\
<0.01 \\
0.02\end{array}$ & $\begin{array}{l}0.48 \\
0.75 \\
1.07 \\
1.14 \\
0.09 \\
0.68 \\
1.54 \\
0.72 \\
\end{array}$ & 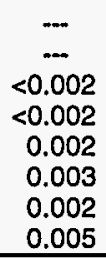 & $\begin{array}{r}<0.02 \\
<0.02 \\
<0.05 \\
0.13 \\
<0.05 \\
<0.02 \\
0.30 \\
<0.02\end{array}$ & $\begin{array}{c}\cdots \\
\cdots . . \\
0.005 \\
<0.002 \\
<0.002 \\
<0.002 \\
<0.002 \\
<0.002 \\
\end{array}$ & $\begin{array}{c}<0.1 \\
\cdots- \\
\cdots \\
-- \\
-- \\
-- \\
0.001\end{array}$ & $\begin{array}{c}\cdots \\
\cdots \\
\cdots \\
\cdots \\
\cdots \\
-. \\
0.001\end{array}$ & $\begin{array}{c}<0.1 \\
<0.1 \\
<0.1 \\
<0.1 \\
0.03 \\
0.02 \\
<0.01 \\
<0.05 \\
\end{array}$ & $\begin{array}{l}-. \\
-. \\
<0.02 \\
<0.01 \\
<0.01 \\
<0.01 \\
<0.01\end{array}$ & $\begin{array}{l}\cdots \\
- \\
- \\
- \\
- \\
\cdots \\
\cdots \\
\cdots \\
--\end{array}$ & $\begin{array}{r}0.01 \\
0.03 \\
0.02 \\
0.03 \\
<0.01 \\
<0.01 \\
0.07 \\
0.01 \\
\end{array}$ \\
\hline
\end{tabular}




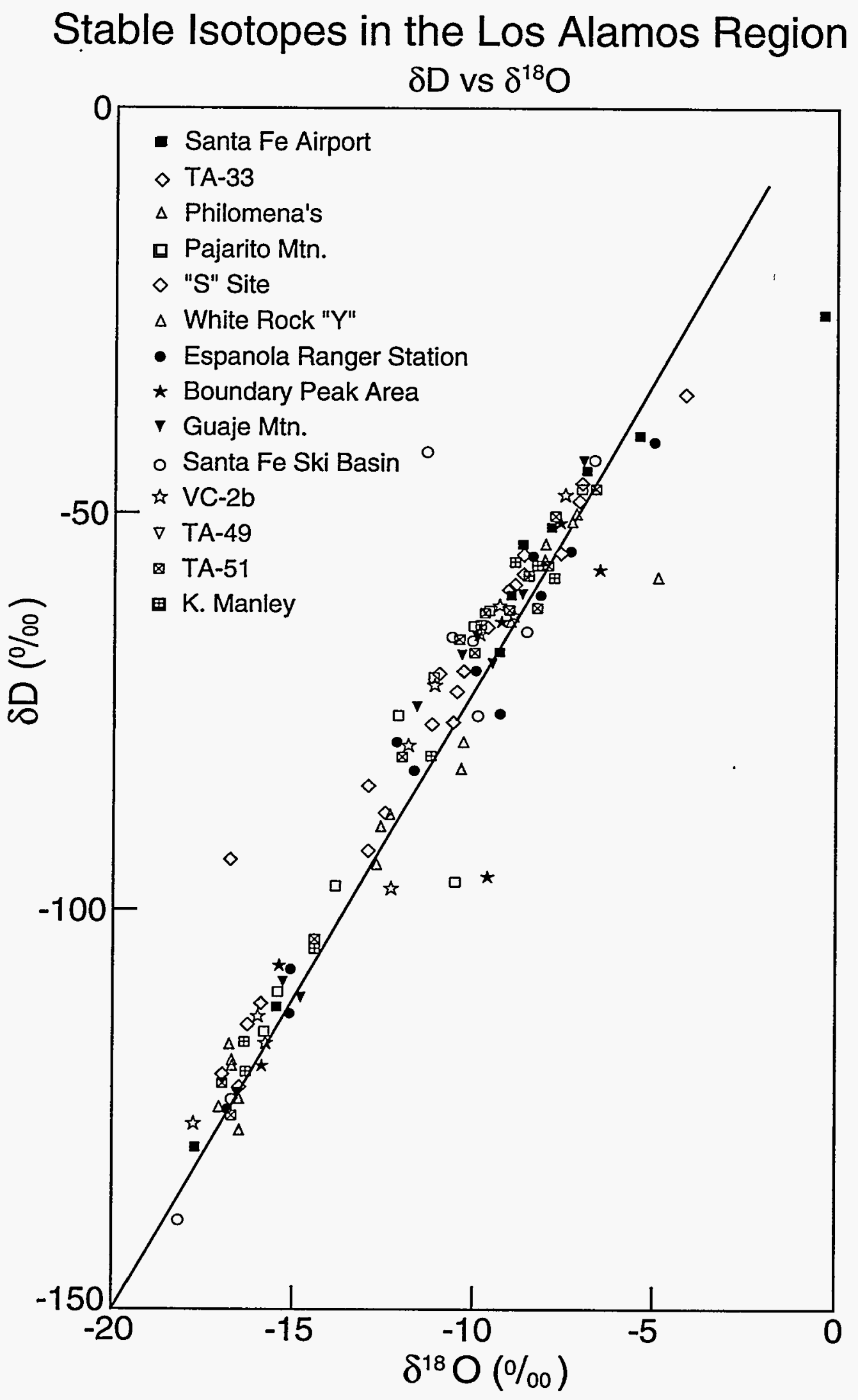

Figure 5: Plot of deuterium versus oxygen-18 for precipitation in the Los Alamos region. The line shown is the World Meteoric Water Line of Craig (1961), $\delta \mathrm{D}=8 \delta^{18} \mathrm{O}+10$. 
Elevation vs Oxygen-18

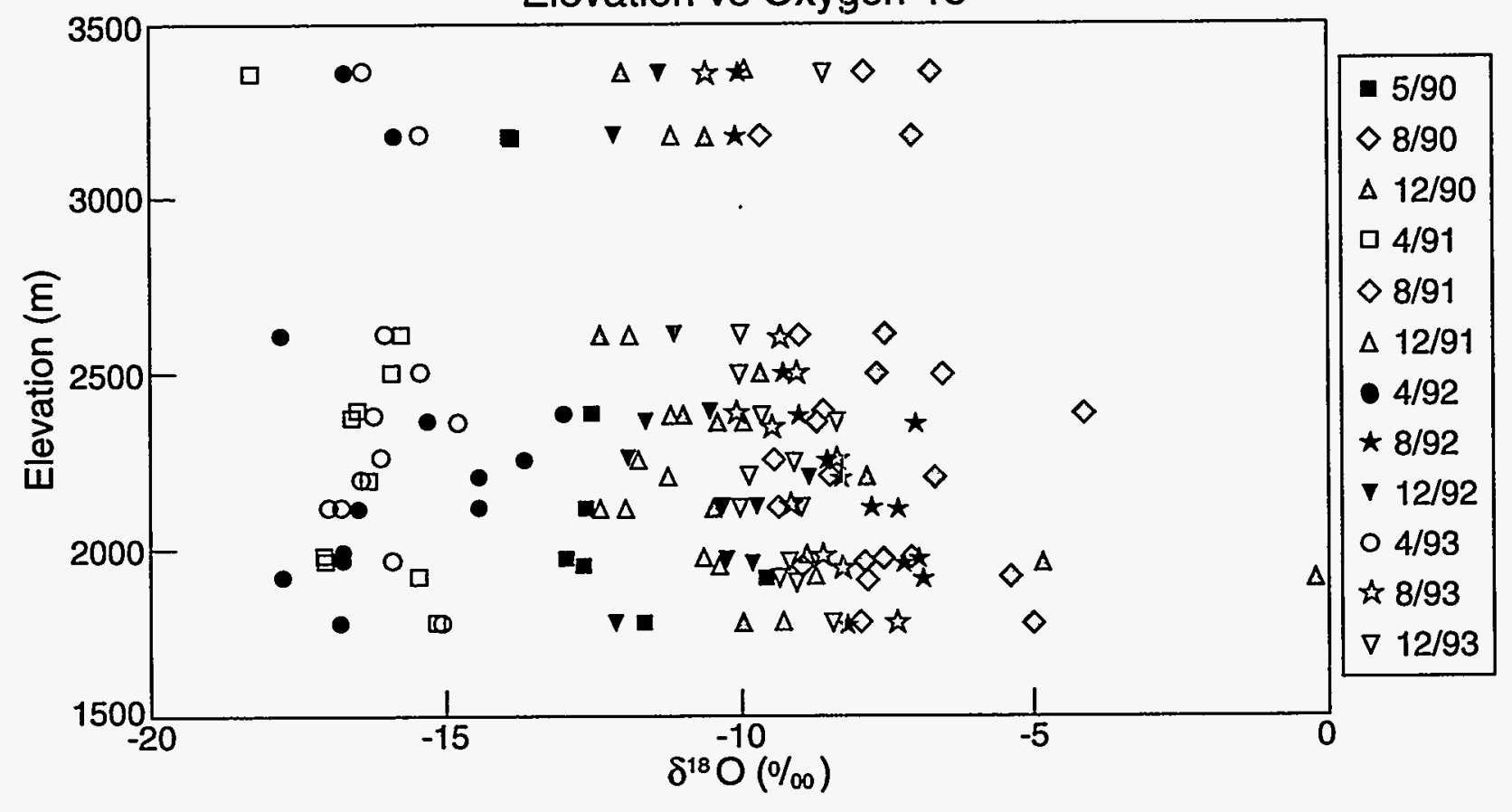

Figure 6: Plot of oxygen-18 versus elevation of sampling stations for precipitation in the Los Alamos region.

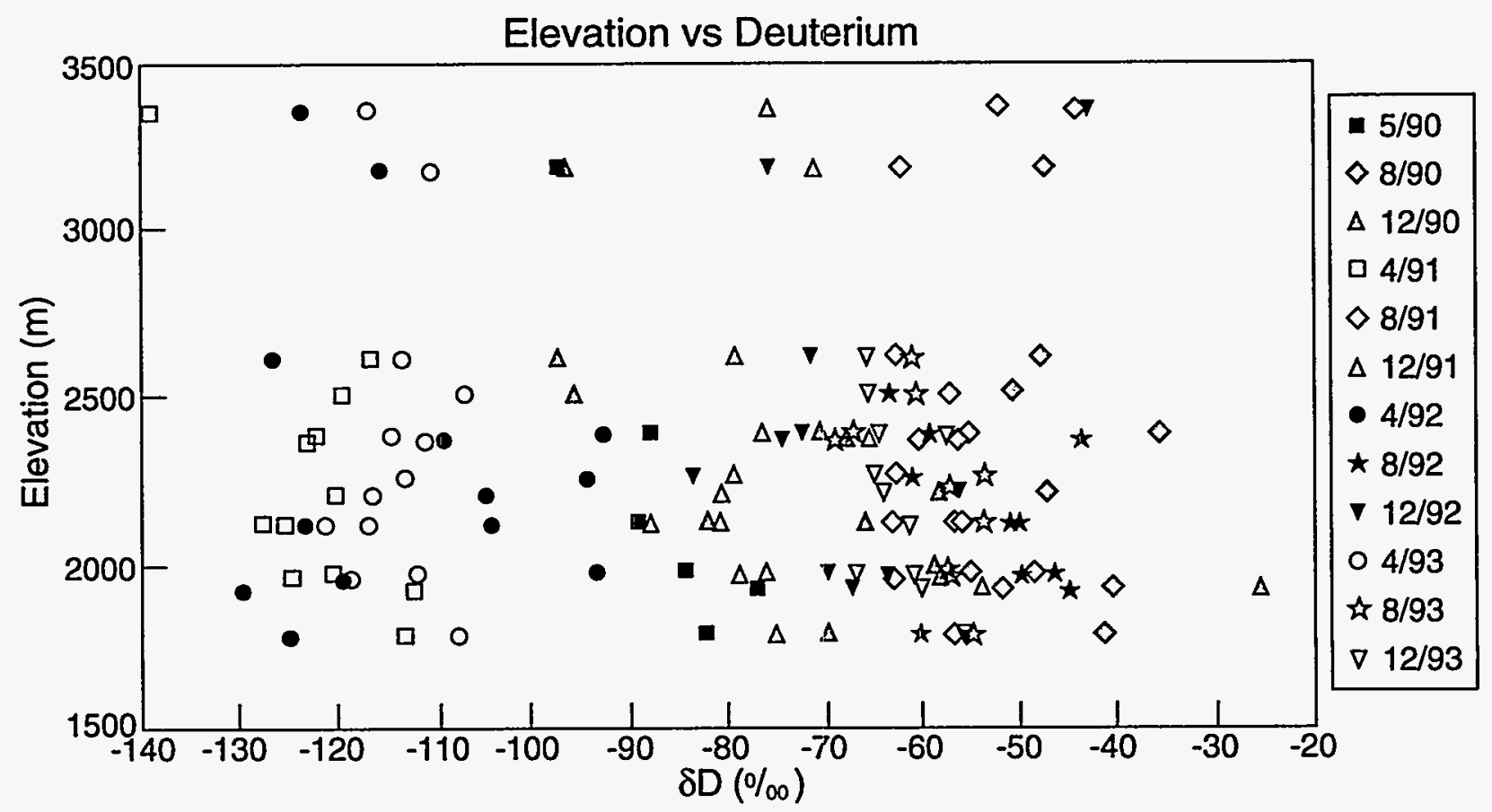

Figure 7: Plot of deuterium versus elevation of sampling stations for precipitation in the Los Alamos region. 
TABLE 4. Isotope Data for Precipitation in the Los Alamos Region, New Mexico (a)

\begin{tabular}{|c|c|c|c|c|c|c|}
\hline Sample \# & Description & Date & $\begin{array}{c}\delta D \\
(\%)\end{array}$ & $\begin{array}{l}\delta^{180} \\
(\% \circ)\end{array}$ & $\begin{array}{c}\text { 3H } \\
\text { (T.U.) }\end{array}$ & $\begin{array}{c}\text { Elevation } \\
\text { (m) }\end{array}$ \\
\hline $\begin{array}{l}\text { WC-1 } \\
\text { WC-9 } \\
\text { WC-28 } \\
\text { WC-46 } \\
\text { WC-51 } \\
\text { WC-67 } \\
\text { WC-80 } \\
\text { WC-97 } \\
\text { WC-113 } \\
\text { WC-146 }\end{array}$ & $\begin{array}{l}\text { Santa Fe Airport } \\
\text { Santa Fe Airport } \\
\text { Santa Fe Airport } \\
\text { Santa Fe Airport } \\
\text { Santa Fe Airport } \\
\text { Santa Fe Airport } \\
\text { Santa Fe Airport } \\
\text { Santa Fe Airport } \\
\text { Santa Fe Airport } \\
\text { Santa Fe Airport }\end{array}$ & $\begin{array}{l}05 / 23 / 90 \\
08 / 09 / 90 \\
11 / 28 / 90 \\
04 / 25 / 91 \\
07 / 29 / 91 \\
12 / 04 / 91 \\
04 / 20 / 92 \\
08 / 14 / 92 \\
12 / 04 / 92 \\
11 / 18 / 93\end{array}$ & $\begin{array}{r}-77.3 \\
-40.5 \\
-25.4 \\
-112.1 \\
-51.7 \\
-53.8 \\
-129.6 \\
-44.7 \\
-67.3 \\
-59.9\end{array}$ & $\begin{array}{l}-9.60 \\
-5.40 \\
-0.29 \\
-15.48 \\
-7.83 \\
-8.72 \\
-17.77 \\
-6.91 \\
-9.35 \\
-9.03\end{array}$ & $\begin{array}{r}11.30 \\
11.80 \\
14.00 \\
9.81 \\
10.30 \\
17.40 \\
10.80 \\
11.80 \\
8.51 \\
7.07\end{array}$ & $\begin{array}{l}1910 \\
1910 \\
1910 \\
1910 \\
1910 \\
1910 \\
1910 \\
1910 \\
1910 \\
1910\end{array}$ \\
\hline $\begin{array}{l}\text { WC-2 } \\
\text { WC-8 } \\
\text { WC-25 } \\
\text { WC-37 } \\
\text { WC-54 } \\
\text { WC-70 } \\
\text { WC-83 } \\
\text { WC-102 } \\
\text { WC-110 } \\
\text { WC-125 } \\
\text { WC-138 } \\
\text { WC-149 }\end{array}$ & $\begin{array}{l}\text { Tech Area-33 } \\
\text { Tech Area-33 } \\
\text { Tech Area-33 } \\
\text { Tech Area-33 } \\
\text { Tech Area-33 } \\
\text { Tech Area-33 } \\
\text { Tech Area-33 } \\
\text { Tech Area-33 } \\
\text { Tech Area-33 } \\
\text { Tech Area-33 } \\
\text { Tech Area-33 } \\
\text { Tech Area-33 }\end{array}$ & $\begin{array}{l}05 / 23 / 90 \\
08 / 09 / 90 \\
11 / 27 / 90 \\
04 / 23 / 91 \\
08 / 01 / 91 \\
12 / 09 / 91 \\
04 / 20 / 92 \\
08 / 20 / 92 \\
12 / 03 / 92 \\
04 / 28 / 93 \\
08 / 09 / 93 \\
12 / 03 / 93\end{array}$ & $\begin{array}{r}-84.2 \\
-55.0 \\
-58.6 \\
-120.4 \\
-48.6 \\
-76.2 \\
-93.6 \\
-46.2 \\
-69.6 \\
-111.8 \\
-57.3 \\
-66.8\end{array}$ & $\begin{array}{c}-12.96 \\
-7.59 \\
-8.84 \\
-17.03 \\
-7.12 \\
-10.62 \\
-16.81 \\
-7.03 \\
-10.29 \\
-15.93 \\
-8.66 \\
-10.24\end{array}$ & $\begin{array}{l}27.20 \\
55.30 \\
36.70 \\
75.30 \\
26.10 \\
18.90 \\
47.40 \\
35.30 \\
29.20 \\
18.70 \\
27.60 \\
17.70\end{array}$ & $\begin{array}{l}1960 \\
1960 \\
1960 \\
1960 \\
1960 \\
1960 \\
1960 \\
1960 \\
1960 \\
1960 \\
1960 \\
1960\end{array}$ \\
\hline $\begin{array}{l}\text { WC-3 } \\
\text { WC-19 } \\
\text { WC-33 } \\
\text { WC-42 } \\
\text { WC-57 } \\
\text { WC-77 } \\
\text { WC-79 } \\
\text { WC-99 } \\
\text { WC-107 } \\
\text { WC-127 } \\
\text { WC-140 } \\
\text { WC-152 }\end{array}$ & $\begin{array}{l}\text { Philomena's } \\
\text { Philomena's } \\
\text { Philomena's } \\
\text { Philomena's } \\
\text { Philomena's } \\
\text { Philomena's } \\
\text { Philomena's } \\
\text { Philomena's } \\
\text { Philomena's } \\
\text { Philomena's } \\
\text { Philomena's } \\
\text { Philomena's }\end{array}$ & $\begin{array}{l}05 / 23 / 90 \\
09 / 07 / 90 \\
12 / 04 / 90 \\
04 / 24 / 91 \\
08 / 05 / 91 \\
12 / 19 / 91 \\
03 / 31 / 92 \\
08 / 14 / 92 \\
12 / 03 / 92 \\
04 / 29 / 93 \\
08 / 13 / 93 \\
11 / 29 / 93\end{array}$ & $\begin{array}{r}-89.2 \\
-55.8 \\
-82.0 \\
-127.5 \\
-62.8 \\
-87.9 \\
-123.4 \\
-50.9 \\
-67.1 \\
-116.7 \\
-53.7 \\
-61.9\end{array}$ & $\begin{array}{c}-12.58 \\
-8.06 \\
-10.38 \\
-16.56 \\
-9.32 \\
-12.36 \\
-16.53 \\
-7.29 \\
-10.18 \\
-16.80 \\
-8.04 \\
-8.99\end{array}$ & $\begin{array}{l}89.60 \\
74.40 \\
80.70 \\
89.60 \\
36.70 \\
45.20 \\
95.50 \\
63.40 \\
70.90 \\
40.20 \\
43.70 \\
45.30\end{array}$ & $\begin{array}{l}2110 \\
2110 \\
2110 \\
2110 \\
2110 \\
2110 \\
2110 \\
2110 \\
2110 \\
2110 \\
2110 \\
2110\end{array}$ \\
\hline $\begin{array}{l}\text { WC-4 } \\
\text { WC-20 } \\
\text { WC-34 } \\
\text { WC-45 } \\
\text { WC-61 } \\
\text { WC-69 } \\
\text { WC-90 } \\
\text { WC-93 } \\
\text { WC-116 } \\
\text { WC-130 } \\
\text { WC-136 } \\
\text { WC-155 }\end{array}$ & $\begin{array}{l}\text { Pajarito Mountain } \\
\text { Pajarito Mountain } \\
\text { Pajarito Mountain } \\
\text { Pajarito Mountain } \\
\text { Pajarito Mountain } \\
\text { Pajarito Mountain } \\
\text { Pajarito Mountain } \\
\text { Pajarito Mountain } \\
\text { Pajarito Mountain } \\
\text { Pajarito Mountain } \\
\text { Pajarito Mountain } \\
\text { Pajarito Mountain }\end{array}$ & $\begin{array}{l}05 / 23 / 90 \\
09 / 07 / 90 \\
12 / 07 / 90 \\
04 / 25 / 91 \\
08 / 06 / 91 \\
12 / 08 / 91 \\
05 / 07 / 92 \\
08 / 10 / 92 \\
12 / 16 / 92 \\
05 / 04 / 93 \\
08 / 09 / 93 \\
02 / 02 / 94\end{array}$ & $\begin{array}{r}-96.8 \\
-47.0 \\
-96.4 \\
-- \\
-62.1 \\
-70.9 \\
-115.2 \\
-64.1 \\
-75.5 \\
-110.1 \\
-64.2 \\
-78.4\end{array}$ & $\begin{array}{l}-13.86 \\
-7.05 \\
-10.53 \\
-- \\
-9.61 \\
-11.11 \\
-15.86 \\
-10.02 \\
-12.08 \\
-15.42 \\
-10.02 \\
-11.59\end{array}$ & $\begin{array}{r}14.20 \\
33.60 \\
9.97 \\
-- \\
15.80 \\
8.50 \\
16.50 \\
18.80 \\
14.30 \\
10.60 \\
21.10 \\
18.20\end{array}$ & $\begin{array}{l}3180 \\
3180 \\
3180 \\
3180 \\
3180 \\
3180 \\
3180 \\
3180 \cdot \\
3180 \\
3180 \\
3180 \\
3180\end{array}$ \\
\hline $\begin{array}{l}\text { WC-5 } \\
\text { WC-15 } \\
\text { WC-31 } \\
\text { WC-41 }\end{array}$ & $\begin{array}{l}\text { "S" Site } \\
\text { "S" Site } \\
\text { "S" Site } \\
\text { "S" Site }\end{array}$ & $\begin{array}{l}05 / 30 / 90 \\
09 / 05 / 90 \\
12 / 04 / 90 \\
04 / 24 / 91\end{array}$ & $\begin{array}{r}-87.9 \\
-35.6 \\
-70.3 \\
-122.2\end{array}$ & $\begin{array}{c}-12.48 \\
-4.14 \\
-11.03 \\
-16.53\end{array}$ & $\begin{array}{l}39.90 \\
58.70 \\
18.60 \\
25.50\end{array}$ & $\begin{array}{l}2380 \\
2380 \\
2380 \\
2380\end{array}$ \\
\hline
\end{tabular}




\begin{tabular}{|c|c|c|c|c|c|c|}
\hline Sample \# & Description & Date & $\begin{array}{l}\delta \mathrm{D} \\
(\% \circ)\end{array}$ & $\begin{array}{l}\delta^{180} \\
(\% \circ)\end{array}$ & $\begin{array}{c}{ }^{3} \mathrm{H} \\
(T . U .)\end{array}$ & $\begin{array}{l}\text { Elevation } \\
(\mathrm{m})\end{array}$ \\
\hline $\begin{array}{l}\text { WC-56 } \\
\text { WC-78 } \\
\text { WC-87 } \\
\text { WC-103 } \\
\text { WC-115 } \\
\text { WC-129 } \\
\text { WC-134 } \\
\text { WC-153 }\end{array}$ & $\begin{array}{l}\text { "S" Site } \\
\text { "S" Site } \\
\text { "S" Site } \\
\text { "S" Site } \\
\text { "S" Site } \\
\text { "S" Site } \\
\text { "S" Site } \\
\text { "S" Site }\end{array}$ & $\begin{array}{l}08 / 05 / 91 \\
12 / 19 / 91 \\
04 / 29 / 92 \\
08 / 20 / 92 \\
12 / 16 / 92 \\
04 / 30 / 93 \\
08 / 05 / 93 \\
11 / 30 / 93\end{array}$ & $\begin{array}{r}-55.2 \\
-76.5 \\
-92.5 \\
-59.1 \\
-72.4 \\
-114.3 \\
-67.2 \\
-64.3\end{array}$ & $\begin{array}{c}-8.61 \\
-11.16 \\
-12.97 \\
-8.96 \\
-10.49 \\
-16.27 \\
-10.08 \\
-9.67\end{array}$ & $\begin{array}{l}22.20 \\
17.70 \\
34.70 \\
28.40 \\
32.20 \\
20.10 \\
13.30 \\
11.20\end{array}$ & $\begin{array}{l}2380 \\
2380 \\
2380 \\
2380 \\
2380 \\
2380 \\
2380 \\
2380\end{array}$ \\
\hline $\begin{array}{l}\text { WC-6 } \\
\text { WC-10 } \\
\text { WC-32 } \\
\text { WC-43 } \\
\text { WC-55 } \\
\text { WC-72 } \\
\text { WC-84 } \\
\text { WC-98 } \\
\text { WC-108 } \\
\text { WC-126 } \\
\text { WC-137 } \\
\text { WC-148 }\end{array}$ & $\begin{array}{l}\text { White Rock "Y" } \\
\text { White Rock "Y" } \\
\text { White Rock "Y" } \\
\text { White Rock "Y" } \\
\text { White Rock "Y" } \\
\text { White Rock "Y" } \\
\text { White Rock "Y" } \\
\text { White Rock "Y" } \\
\text { White Rock "Y" } \\
\text { White Rock "Y" } \\
\text { White Rock "Y" } \\
\text { White Rock "Y" }\end{array}$ & $\begin{array}{l}05 / 30 / 90 \\
08 / 09 / 90 \\
12 / 04 / 90 \\
04 / 24 / 91 \\
08 / 01 / 91 \\
12 / 10 / 91 \\
04 / 28 / 92 \\
08 / 14 / 92 \\
12 / 03 / 92 \\
04 / 29 / 93 \\
08 / 09 / 93 \\
11 / 29 / 93\end{array}$ & $\begin{array}{r}-94.1 \\
-57.6 \\
-57.8 \\
-124.5 \\
-63.2 \\
-78.7 \\
-119.3 \\
-49.9 \\
-63.5 \\
-118.9 \\
-57.0 \\
-60.7\end{array}$ & $\begin{array}{l}-12.70 \\
-7.86 \\
-4.87 \\
-17.06 \\
-9.05 \\
-10.37 \\
-16.76 \\
-7.18 \\
-9.80 \\
-16.76 \\
-8.28 \\
-9.12\end{array}$ & $\begin{array}{l}141.00 \\
51.90 \\
82.50 \\
117.00 \\
24.40 \\
22.20 \\
34.00 \\
30.50 \\
28.20 \\
15.70 \\
25.10 \\
31.40\end{array}$ & $\begin{array}{l}1950 \\
1950 \\
1950 \\
1950 \\
1950 \\
1950 \\
1950 \\
1950 \\
1950 \\
1950 \\
1950 \\
1950\end{array}$ \\
\hline $\begin{array}{l}\text { WC-7 } \\
\text { WC-14 } \\
\text { WC-30 } \\
\text { WC-44 } \\
\text { WC-58 } \\
\text { WC-71 } \\
\text { WC-86 } \\
\text { WC-101 } \\
\text { WC-114 } \\
\text { WC-128 } \\
\text { WC-133 } \\
\text { WC-147 }\end{array}$ & $\begin{array}{l}\text { Espanola Ranger Station } \\
\text { Espanola Ranger Station } \\
\text { Espanola Ranger Station } \\
\text { Espanola Ranger Station } \\
\text { Espanola Ranger Station } \\
\text { Espanola Ranger Station } \\
\text { Espanola Ranger Station } \\
\text { Espanola Ranger Station } \\
\text { Espanola Ranger Station } \\
\text { Espanola Ranger Station } \\
\text { Espanola Ranger Station } \\
\text { Espanola Ranger Station }\end{array}$ & $\begin{array}{l}05 / 30 / 90 \\
09 / 05 / 90 \\
11 / 28 / 90 \\
04 / 24 / 91 \\
08 / 05 / 91 \\
12 / 10 / 91 \\
04 / 29 / 92 \\
08 / 20 / 92 \\
12 / 16 / 92 \\
04 / 30 / 93 \\
08 / 05 / 93 \\
11 / 29 / 93\end{array}$ & $\begin{array}{r}-82.4 \\
-41.3 \\
-69.7 \\
-112.9 \\
-56.4 \\
-75.3 \\
-125.1 \\
-60.2 \\
-78.9 \\
-107.4 \\
-54.7 \\
-55.3\end{array}$ & $\begin{array}{l}-11.67 \\
-5.01 \\
-9.99 \\
-15.16 \\
-7.99 \\
-9.29 \\
-16.85 \\
-8.22 \\
-12.11 \\
-15.10 \\
-7.35 \\
-8.41\end{array}$ & $\begin{array}{l}37.10 \\
30.60 \\
14.80 \\
37.00 \\
13.20 \\
12.10 \\
12.70 \\
16.20 \\
11.50 \\
10.00 \\
13.80 \\
10.90\end{array}$ & $\begin{array}{l}1775 \\
1775 \\
1775 \\
1775 \\
1775 \\
1775 \\
1775 \\
1775 \\
1775 \\
1775 \\
1775 \\
1775\end{array}$ \\
\hline $\begin{array}{l}\text { WC-11 } \\
\text { WC-23 } \\
\text { WC-36 } \\
\text { WC-49 } \\
\text { WC-94 } \\
\text { WC-124 } \\
\text { WC-131 } \\
\text { WC-143 }\end{array}$ & $\begin{array}{l}\text { Boundary Peak Trail } \\
\text { Boundary Peak Trail } \\
\text { Boundary Peak Trail } \\
\text { Boundary Peak Trail } \\
\text { Boundary Peak Trail } \\
\text { Boundary Peak Trail } \\
\text { Boundary Peak Trail } \\
\text { Boundary Peak Trail }\end{array}$ & $\begin{array}{l}09 / 04 / 90 \\
11 / 26 / 90 \\
04 / 23 / 91 \\
07 / 29 / 91 \\
08 / 10 / 92 \\
04 / 28 / 93 \\
08 / 03 / 93 \\
11 / 16 / 93\end{array}$ & $\begin{array}{r}-50.6 \\
-95.6 \\
-119.4 \\
-57.0 \\
-63.3 \\
-106.9 \\
-60.6 \\
-65.4\end{array}$ & $\begin{array}{r}-7.67 \\
-9.65 \\
-15.92 \\
-6.55 \\
-9.26 \\
-15.43 \\
-9.15 \\
-9.97\end{array}$ & $\begin{array}{r}20.80 \\
10.60 \\
27.00 \\
12.50 \\
23.00 \\
15.00 \\
14.40 \\
9.03\end{array}$ & $\begin{array}{l}2500 \\
2500 \\
2500 \\
2500 \\
2500 \\
2500 \\
2500 \\
2500\end{array}$ \\
\hline $\begin{array}{l}\text { WC-12 } \\
\text { WC-24 } \\
\text { WC-39 } \\
\text { WC-50 } \\
\text { WC-76 } \\
\text { WC-85 } \\
\text { WC-92 } \\
\text { WC-106 } \\
\text { WC-119 } \\
\text { WC-135 } \\
\text { WC-144 }\end{array}$ & $\begin{array}{l}\text { West of Guaje Mtn. } \\
\text { West of Guaje Mtn. } \\
\text { West of Guaje Mtn. } \\
\text { West of Guaje Mtn. } \\
\text { West of Guaje Mtn. } \\
\text { West of Guaje Mtn. } \\
\text { West of Guaje Mtn. } \\
\text { West of Guaje Mtn. } \\
\text { West of Guaje Mtn. } \\
\text { Westof Guaje Mtn. } \\
\text { West of Guaje Mtn. }\end{array}$ & $\begin{array}{l}09 / 04 / 90 \\
11 / 27 / 90 \\
04 / 23 / 91 \\
07 / 29 / 91 \\
12 / 18 / 91 \\
04 / 29 / 92 \\
08 / 10 / 92 \\
12 / 02 / 92 \\
04 / 23 / 93 \\
08 / 06 / 93 \\
11 / 17 / 93\end{array}$ & $\begin{array}{r}-56.3 \\
-67.7 \\
-122.9 \\
-60.1 \\
-65.5 \\
-109.0 \\
-43.5 \\
-74.4 \\
-110.9 \\
-68.8 \\
-56.8\end{array}$ & $\begin{array}{r}-8.73 \\
-10.36 \\
-16.60 \\
-8.68 \\
-9.95 \\
-15.33 \\
-6.98 \\
-11.59 \\
-14.80 \\
-9.52 \\
-8.38\end{array}$ & $\begin{array}{l}47.30 \\
25.60 \\
48.10 \\
24.60 \\
31.30 \\
87.10 \\
30.10 \\
32.40 \\
51.30 \\
23.50 \\
19.70\end{array}$ & $\begin{array}{l}2360 \\
2360 \\
2360 \\
2360 \\
2360 \\
2360 \\
2360 \\
2360 \\
2360 \\
2360 \\
2360\end{array}$ \\
\hline
\end{tabular}




\begin{tabular}{|c|c|c|c|c|c|c|}
\hline Sample \# & Description & Date & $\begin{array}{c}\delta D \\
(\%)\end{array}$ & $\begin{array}{c}\delta^{18} 0 \\
(\%)\end{array}$ & $\begin{array}{c}3 \mathrm{H} \\
\text { (T.U.) }\end{array}$ & $\begin{array}{l}\text { Elevation } \\
\text { (m) }\end{array}$ \\
\hline $\begin{array}{l}\text { WC-13 } \\
\text { WC-29 } \\
\text { WC-47 } \\
\text { WC-52 } \\
\text { WC-66 } \\
\text { WC-81 } \\
\text { WC-96 } \\
\text { WC-112 } \\
\text { WC-123 } \\
\text { WC-132 } \\
\text { WC-145 }\end{array}$ & $\begin{array}{l}\text { Santa Fe Ski Basin } \\
\text { Santa Fe Ski Basin } \\
\text { Santa Fe Ski Basin } \\
\text { Santa Fe Ski Basin } \\
\text { Santa Fe Ski Basin } \\
\text { Santa Fe Ski Basin } \\
\text { Santa Fe Ski Basin } \\
\text { Santa Fe Ski Basin } \\
\text { Santa Fe Ski Basin } \\
\text { Santa Fe Ski Basin } \\
\text { Santa Fe Ski Basin }\end{array}$ & $\begin{array}{l}09 / 05 / 90 \\
11 / 28 / 90 \\
04 / 25 / 91 \\
07 / 29 / 91 \\
12 / 04 / 91 \\
04 / 20 / 92 \\
08 / 14 / 92 \\
12 / 04 / 92 \\
04 / 28 / 93 \\
08 / 04 / 93 \\
11 / 18 / 93\end{array}$ & $\begin{array}{r}-43.5 \\
-80.0 \\
-138.8 \\
-51.7 \\
-75.6 \\
-123.5 \\
-65.7 \\
-42.4 \\
-116.5 \\
-65.6 \\
-64.8\end{array}$ & $\begin{array}{r}-6.73 \\
-11.98 \\
-18.25 \\
-7.83 \\
-9.90 \\
-16.68 \\
-10.00 \\
-11.34 \\
-16.42 \\
-10.55 \\
-8.56\end{array}$ & $\begin{array}{r}15.00 \\
7.92 \\
14.60 \\
9.61 \\
10.60 \\
10.30 \\
13.10 \\
8.37 \\
8.60 \\
15.00 \\
7.66\end{array}$ & $\begin{array}{l}3360 \\
3360 \\
3360 \\
3360 \\
3360 \\
3360 \\
3360 \\
3360 \\
3360 \\
3360 \\
3360\end{array}$ \\
\hline $\begin{array}{l}\text { WC-16 } \\
\text { WC-21 } \\
\text { WC-22 } \\
\text { WC-35 } \\
\text { WC-48 } \\
\text { WC-68 } \\
\text { WC-89 } \\
\text { WC-105 } \\
\text { WC-118 } \\
\text { WC-130a } \\
\text { WC-142 }\end{array}$ & $\begin{array}{l}\text { CSDP corehole VC-2B } \\
\text { CSDP corehole VC-2B } \\
\text { CSDP corehole VC-2B } \\
\text { CSDP corehole VC-2B } \\
\text { CSDP corehole VC-2B } \\
\text { CSDP corehole VC-2B } \\
\text { CSDP corehole VC-2B } \\
\text { CSDP corehole VC-2B } \\
\text { CSDP corehole VC-2B } \\
\text { CSDP corehole VC-2B } \\
\text { CSDP corehole VC-2B }\end{array}$ & $\begin{array}{l}09 / 06 / 90 \\
10 / 24 / 90 \\
11 / 26 / 90 \\
04 / 23 / 91 \\
07 / 29 / 91 \\
12 / 05 / 91 \\
05 / 01 / 92 \\
12 / 02 / 92 \\
04 / 21 / 93 \\
08 / 03 / 93 \\
11 / 16 / 93\end{array}$ & $\begin{array}{r}-47.6 \\
-59.8 \\
-97.1 \\
-116.4 \\
-62.4 \\
-79.2 \\
-126.4 \\
-71.3 \\
-113.1 \\
-61.2 \\
-65.6\end{array}$ & $\begin{array}{c}-7.51 \\
-9.09 \\
-12.33 \\
-15.79 \\
-8.99 \\
-11.86 \\
-17.80 \\
-11.10 \\
-16.02 \\
-9.28 \\
-9.96\end{array}$ & $\begin{array}{r}15.30 \\
-. \\
8.71 \\
8.24 \\
11.40 \\
10.00 \\
7.43 \\
7.42 \\
5.85 \\
12.00 \\
6.54\end{array}$ & $\begin{array}{l}2610 \\
2610 \\
2610 \\
2610 \\
2610 \\
2610 \\
2610 \\
2610 \\
2610 \\
2610 \\
2610\end{array}$ \\
\hline $\begin{array}{l}\text { WC-17 } \\
\text { WC-26 } \\
\text { WC-38 } \\
\text { WC-53 } \\
\text { WC-73 } \\
\text { WC-82 } \\
\text { WC-95 } \\
\text { WC-111 } \\
\text { WC-121 } \\
\text { WC-150 }\end{array}$ & $\begin{array}{l}\text { Tech Area-49 } \\
\text { Tech Area-49 } \\
\text { Tech Area-49 } \\
\text { Tech Area-49 } \\
\text { Tech Area-49 } \\
\text { Tech Area-49 } \\
\text { Tech Area-49 } \\
\text { Tech Area-49 } \\
\text { Tech Area-49 } \\
\text { Tech Area-49 }\end{array}$ & $\begin{array}{l}09 / 06 / 90 \\
11 / 27 / 90 \\
04 / 23 / 91 \\
08 / 01 / 91 \\
12 / 16 / 91 \\
04 / 28 / 92 \\
08 / 10 / 92 \\
12 / 03 / 92 \\
04 / 23 / 93 \\
11 / 29 / 93\end{array}$ & $\begin{array}{r}-47.0 \\
-58.0 \\
-120.2 \\
-57.5 \\
-80.6 \\
-104.7 \\
-56.6 \\
-56.1 \\
-116.5 \\
-63.8\end{array}$ & $\begin{array}{c}-6.67 \\
-7.84 \\
-16.35 \\
-8.44 \\
-11.24 \\
-14.46 \\
-8.32 \\
-8.85 \\
-16.42 \\
-9.85\end{array}$ & $\begin{array}{l}64.50 \\
20.80 \\
42.10 \\
20.40 \\
17.60 \\
49.00 \\
33.70 \\
25.60 \\
22.70 \\
14.80\end{array}$ & $\begin{array}{l}2200 \\
2200 \\
2200 \\
2200 \\
2200 \\
2200 \\
2200 \\
2200 \\
2200 \\
2200\end{array}$ \\
\hline $\begin{array}{l}\text { WC-18 } \\
\text { WC-27 } \\
\text { WC-40 } \\
\text { WC-59 } \\
\text { WC-75 } \\
\text { WC-88 } \\
\text { WC-100 } \\
\text { WC-109 } \\
\text { WC-122 } \\
\text { WC-139 } \\
\text { WC-151 }\end{array}$ & $\begin{array}{l}\text { Tech Area-51 } \\
\text { Tech Area-51 } \\
\text { Tech Area-51 } \\
\text { Tech Area-51 } \\
\text { Tech Area-51 } \\
\text { Tech Area-51 } \\
\text { Tech Area-51 } \\
\text { Tech Area-51 } \\
\text { Tech Area-51 } \\
\text { Tech Area-51 } \\
\text { Tech Area-51 }\end{array}$ & $\begin{array}{l}09 / 06 / 90 \\
11 / 27 / 90 \\
04 / 24 / 91 \\
08 / 05 / 91 \\
12 / 17 / 91 \\
04 / 29 / 92 \\
08 / 14 / 92 \\
12 / 03 / 92 \\
04 / 23 / 93 \\
08 / 13 / 93 \\
11 / 29 / 93\end{array}$ & $\begin{array}{r}-56.4 \\
-65.8 \\
-125.3 \\
-61.8 \\
-80.6 \\
-104.0 \\
-50.3 \\
-62.3 \\
-121.5 \\
-62.0 \\
-67.5\end{array}$ & $\begin{array}{c}-8.01 \\
-10.43 \\
-16.81 \\
-8.29 \\
-11.98 \\
-14.45 \\
-7.81 \\
-9.71 \\
-17.01 \\
-9.16 \\
-10.03\end{array}$ & $\begin{array}{l}89.40 \\
26.50 \\
63.40 \\
31.90 \\
12.10 \\
58.40 \\
43.10 \\
40.40 \\
25.20 \\
30.00 \\
21.10\end{array}$ & $\begin{array}{l}2110 \\
2110 \\
2110 \\
2110 \\
2110 \\
2110 \\
2110 \\
2110 \\
2110 \\
2110 \\
2110\end{array}$ \\
\hline $\begin{array}{l}\text { WC-60 } \\
\text { WC-74 } \\
\text { WC-91 } \\
\text { WC-104 } \\
\text { WC-117 } \\
\text { WC-120 } \\
\text { WC-141 } \\
\text { WC-154 }\end{array}$ & $\begin{array}{l}\text { Kim Manley's House } \\
\text { Kim Manley's House } \\
\text { Kim Manley's House } \\
\text { Kim Manley's House } \\
\text { Kim Manley's House } \\
\text { Kim Manley's House } \\
\text { Kim Manley's House } \\
\text { Kim Manley's House }\end{array}$ & $\begin{array}{l}08 / 05 / 91 \\
12 / 15 / 91 \\
05 / 27 / 92 \\
08 / 20 / 92 \\
12 / 18 / 92 \\
04 / 23 / 93 \\
08 / 13 / 93 \\
11 / 29 / 93\end{array}$ & $\begin{array}{r}-62.8 \\
-79.5 \\
-94.2 \\
-61.1 \\
-83.5 \\
-112.9 \\
-53.6 \\
-64.8\end{array}$ & $\begin{array}{l}-9.41 \\
-11.73 \\
-13.65 \\
-8.50 \\
-11.89 \\
-16.12 \\
-8.39 \\
-9.06\end{array}$ & $\begin{array}{c}25.10 \\
36.80 \\
81.20 \\
49.20 \\
115.90 \\
49.10 \\
23.40 \\
23.10\end{array}$ & $\begin{array}{l}2250 \\
2250 \\
2250 \\
2250 \\
2250 \\
2250 \\
2250 \\
2250\end{array}$ \\
\hline
\end{tabular}

(a) Stable isotope analyses from M. Colucci; Southern Methodist University Tritium analyses from H. Gote Ostlund; University of Miami 
TRITIUM IN RAIN, FEB.-MAY 1990
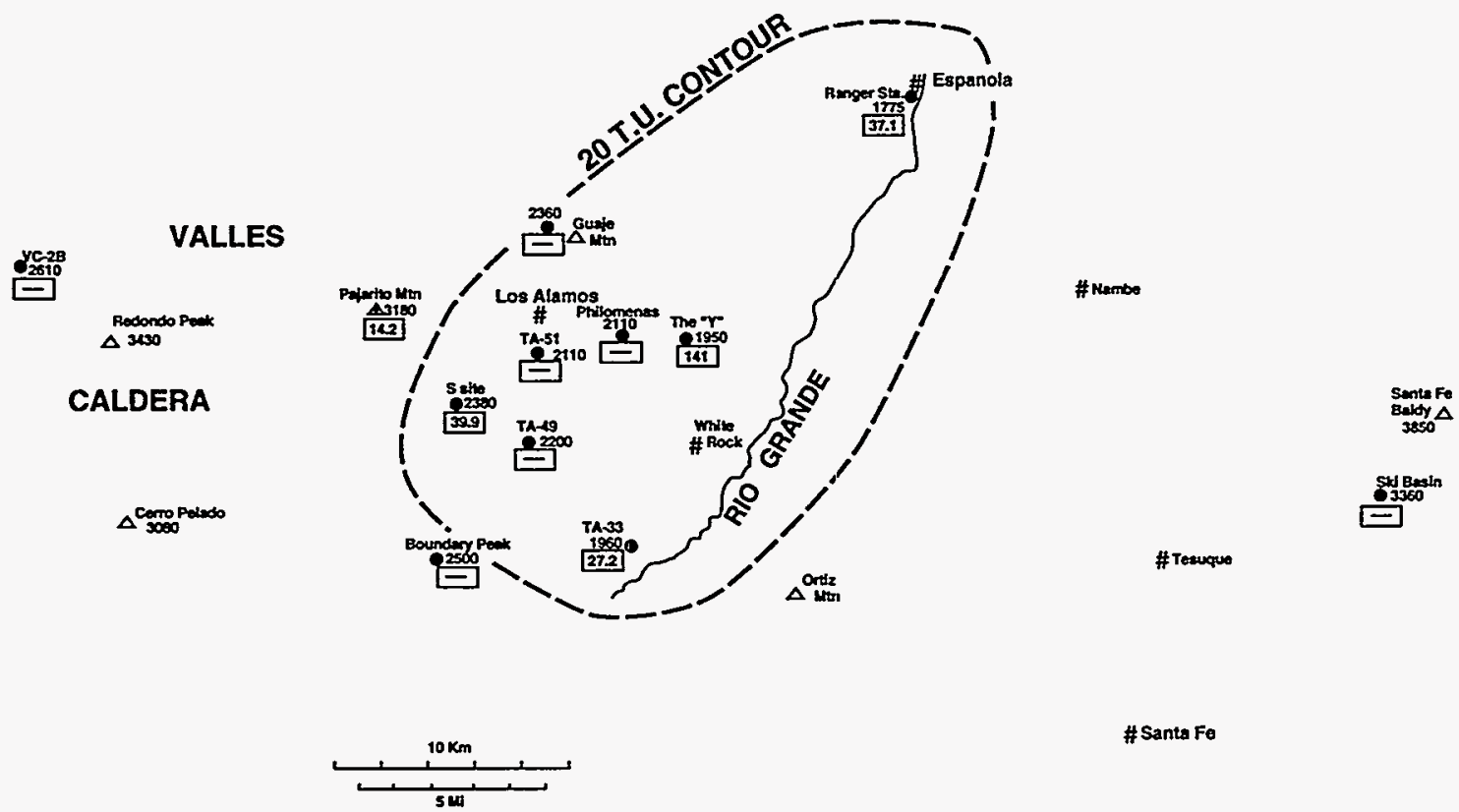

[11.3 • Alpport

Figure 8: Map of sampling stations showing area of anomalous tritium for Feb.-May 1990. Numbers shown in boxes are tritium values in T.U. Adjacent numbers are station elevations in meters. These symbols are used in Figs. 9 to 23.

TRITIUMIN RAIN,_MAY-SEPT. 1990
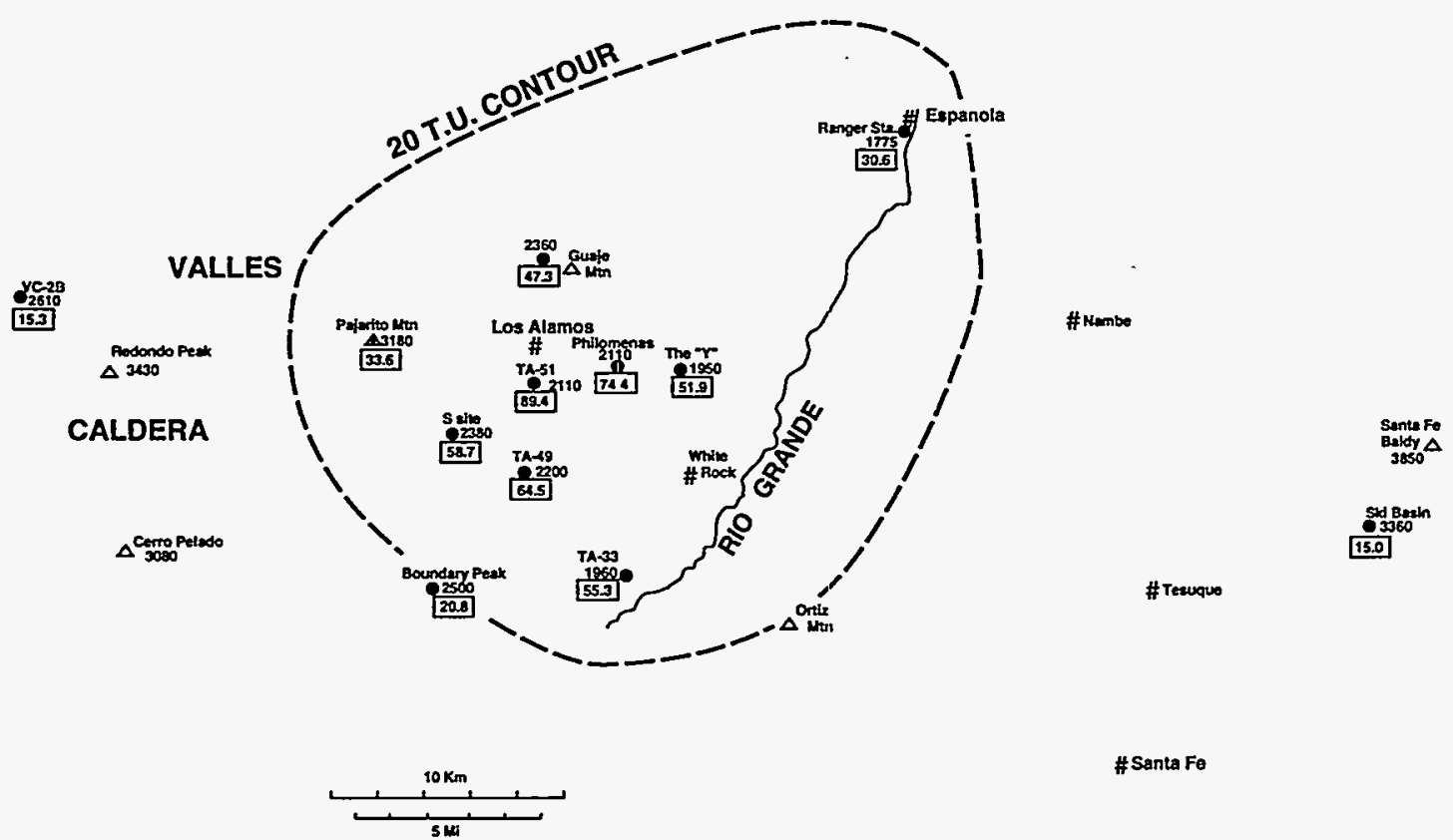

\# Santa Fe

[11.8] Aipon

Figure 9: Map of sampling stations showing area of anomalous tritium for May-Sept. 1990. 


\section{TRITIUM IN RAIN, SEPT.-DEC. 1990}
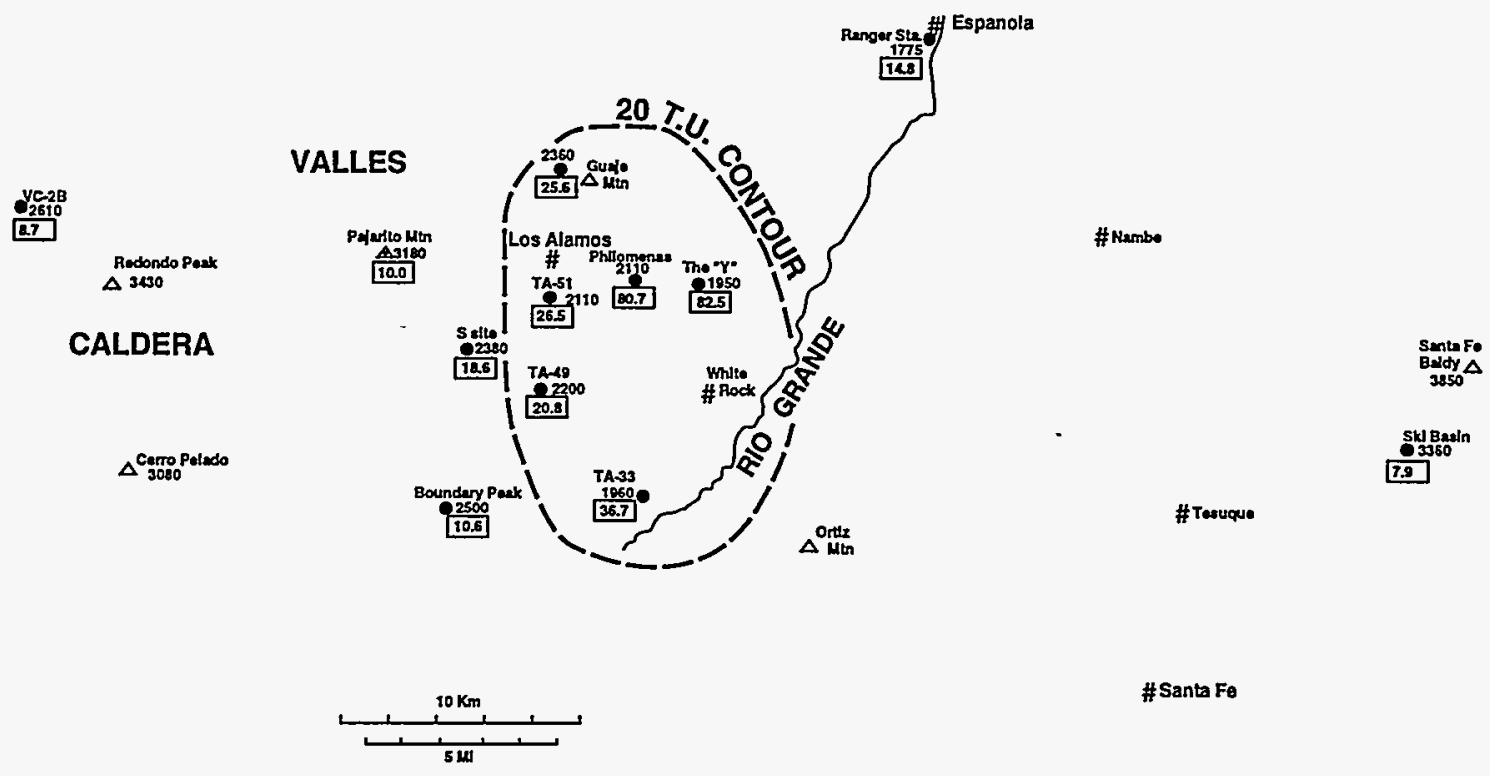

140 - Anport

Figure 10: Map of sampling stations showing area of anomalous tritium for Sept.- Dec. 1990.

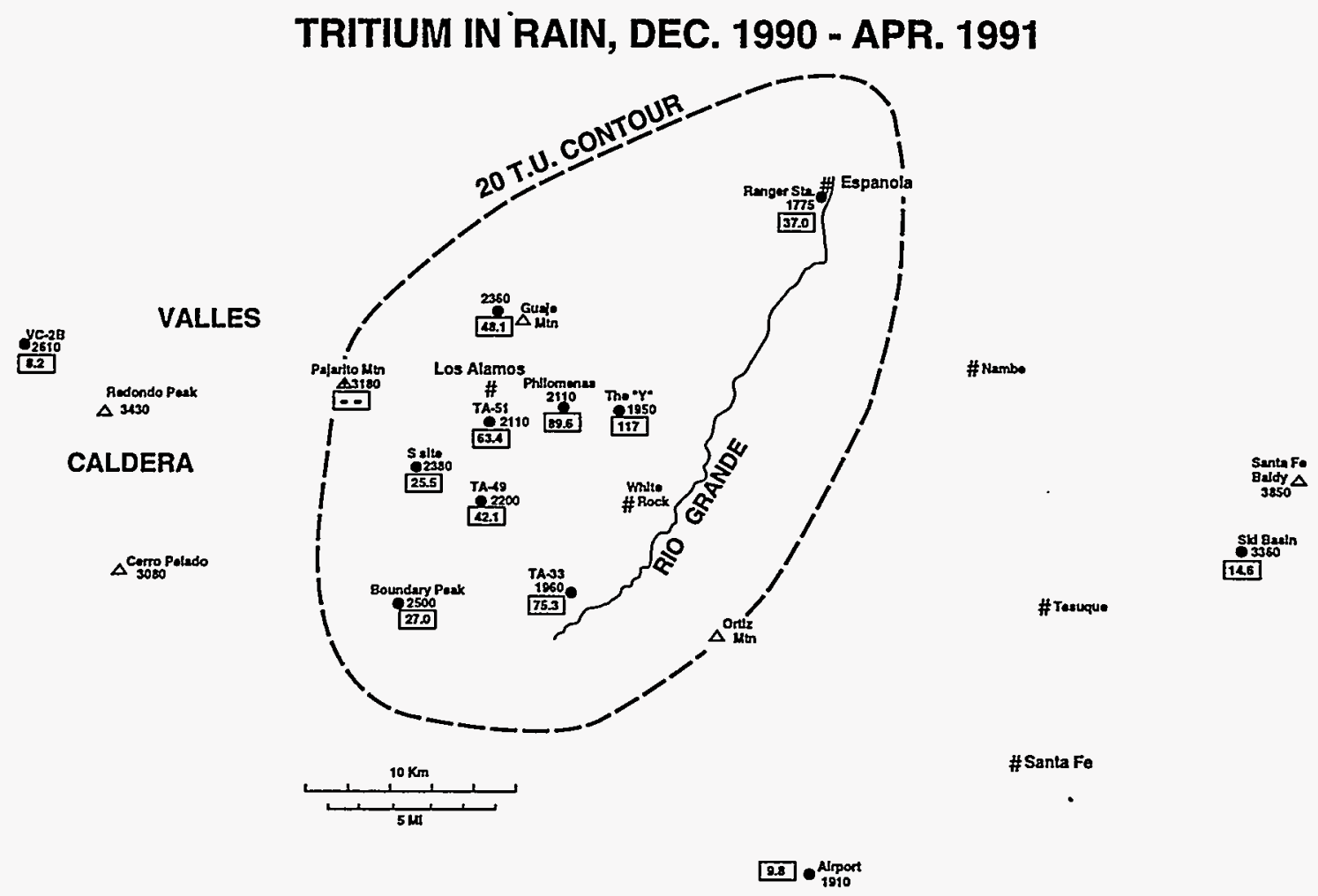

Figure 11: Map of sampling stations showing area of anomalous tritium for Dec. 1990 - April 1991. 


\section{TRITIUM IN RAIN, APRIL-AUG. 1991}
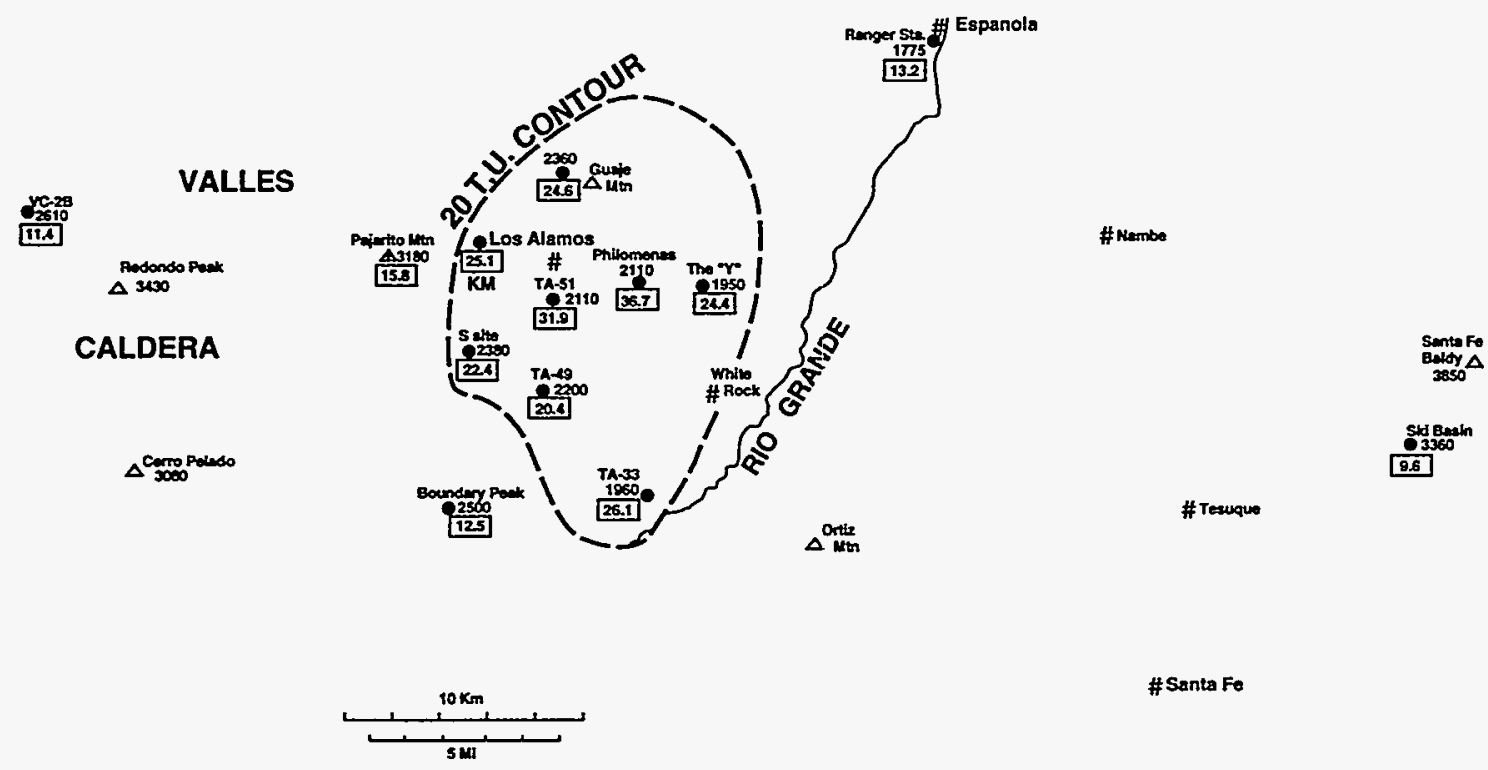

[102] Apront

Figure 12: Map of sampling stations showing area of anomalous tritium for April-Aug. 1991.

\section{TRITIUM IN RAIN, AUG. - DEC. 1991}
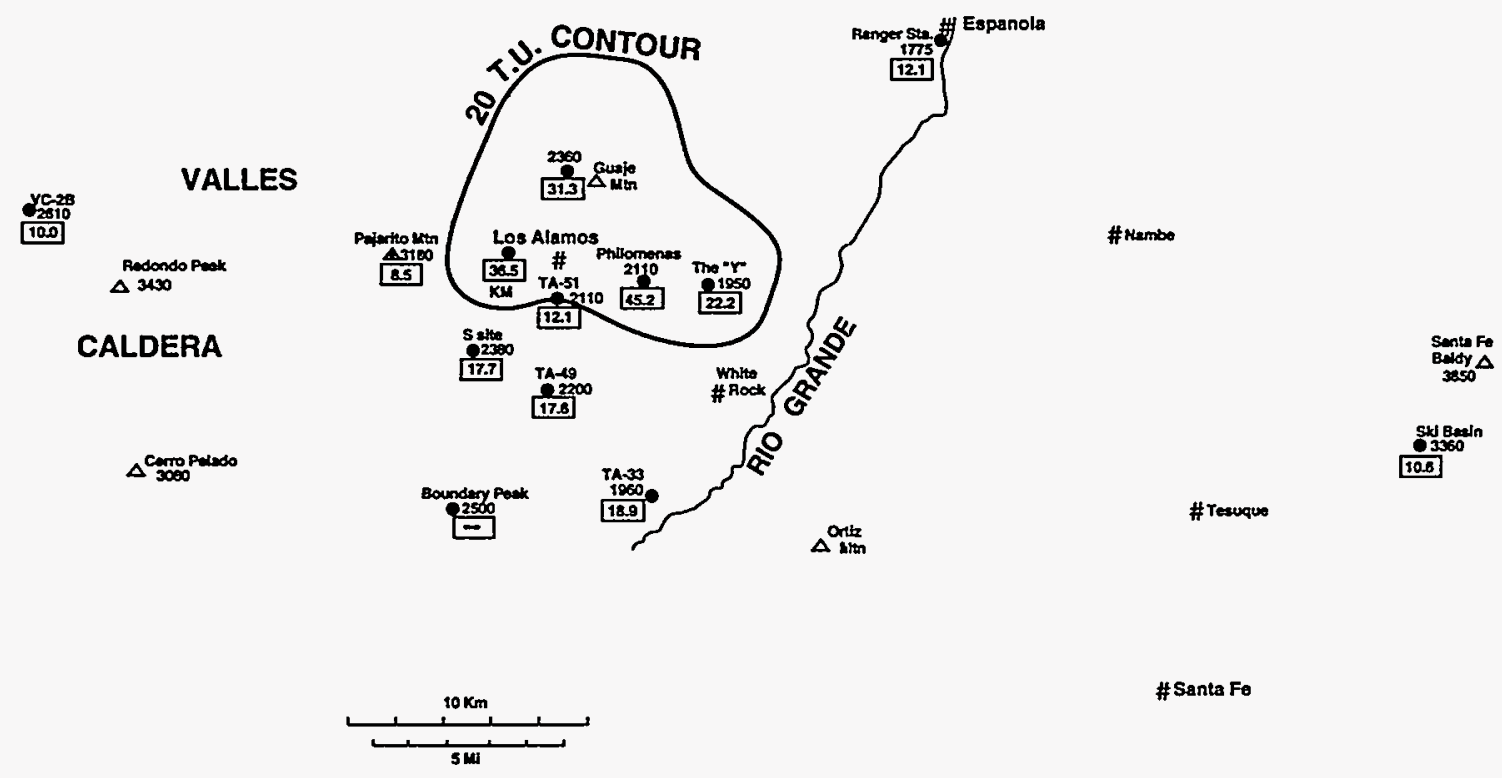

\#Santa Fo

[17.4. $\bullet$ Alport

Figure 13: Map of sampling stations showing area of anomalous tritium for Aug.-Dec. 1991. 


\section{TRITIUM IN RAIN, DEC. 91 - APR. 92}

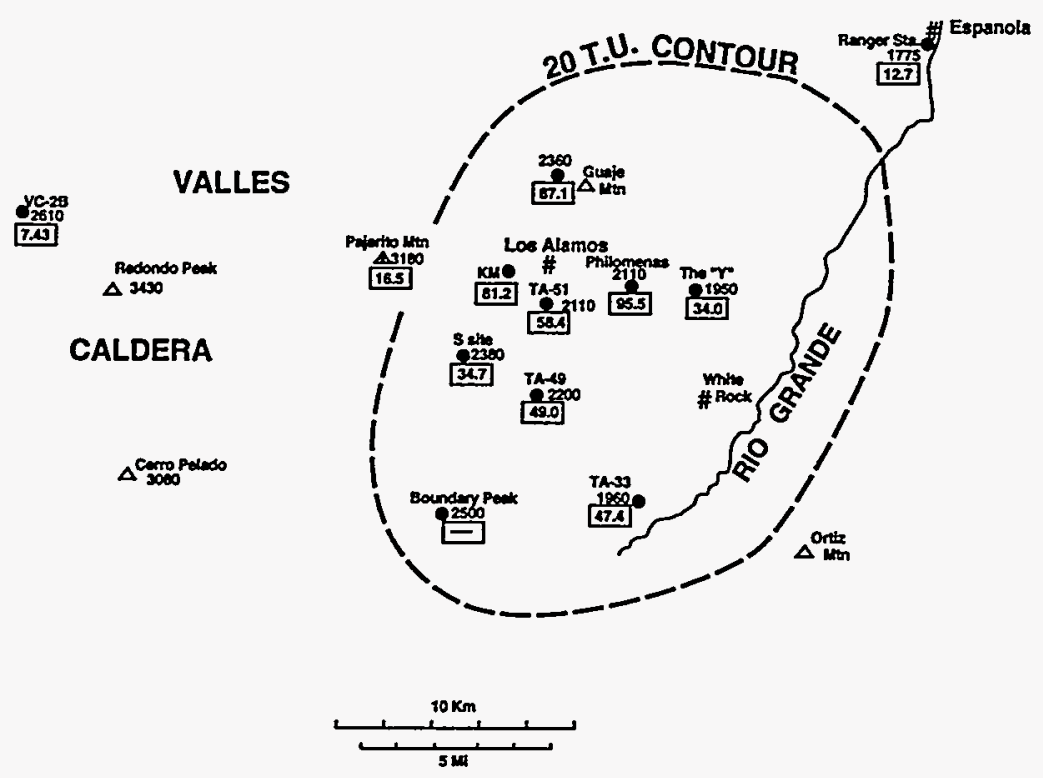

\# Nomon

10.08 Arpon

Figure 14: Map of sampling stations showing area of anomalous tritium for Dec.1991-April 1992.

TRITIUM IN RAIN, APR. - AUG. 1992

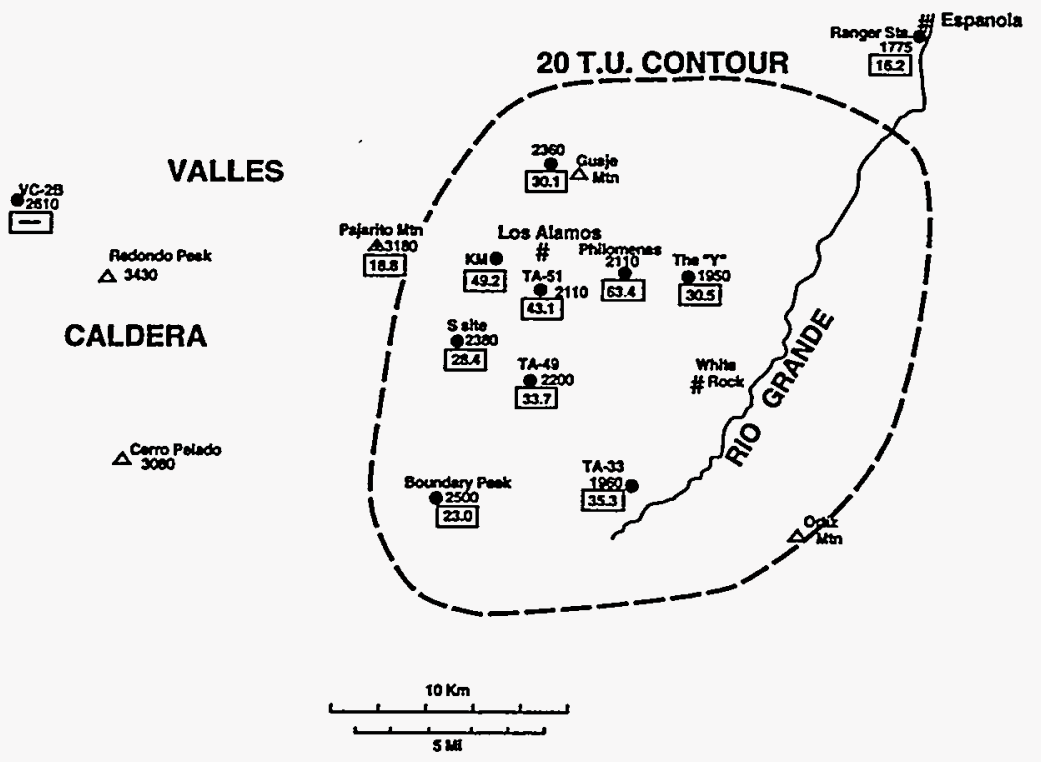

\#Nienbe

\#Senta Fo

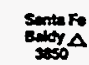

100

* Tomoure 
TRITIUM IN RAIN, AUG. 1992 - DEC. 1992
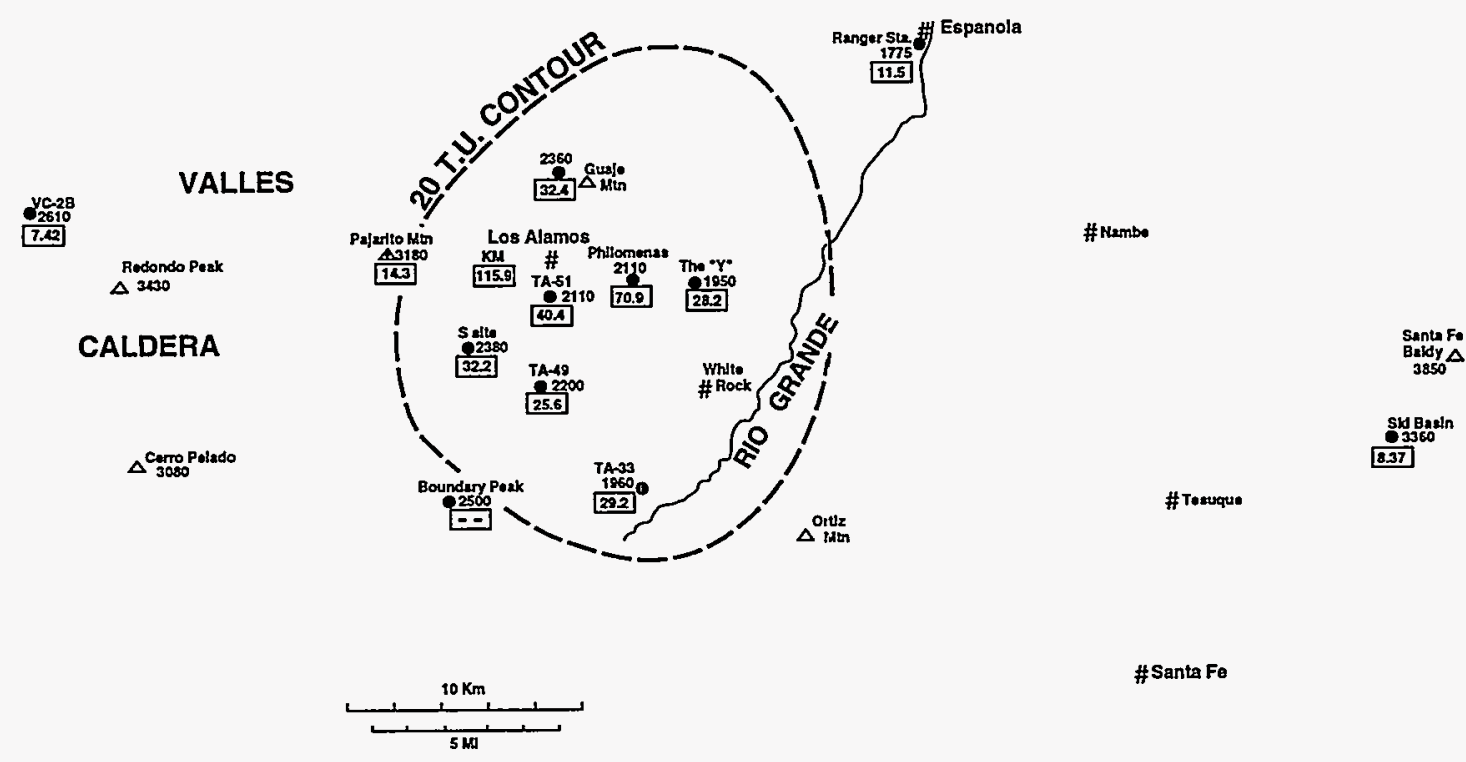

\#Santa Fo

8.51 Alport

Figure 16: Map of sampling stations showing area of anomalous tritium for Aug.-Dec. 1992.

\section{TRITIUM IN RAIN, DEC. 1992 - APR. 1993}
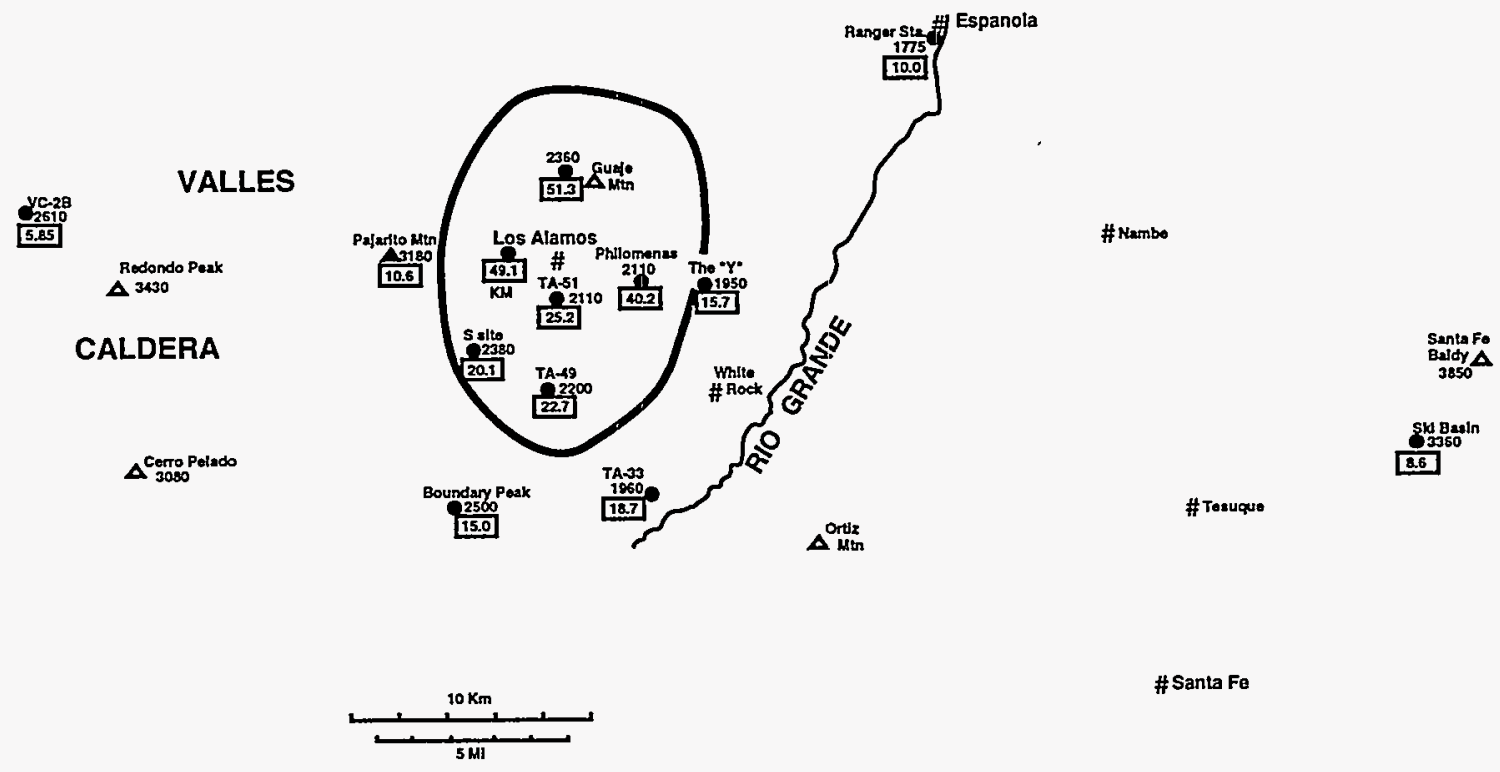

$$
\left[\begin{array}{c}
\text { Alpon } \\
1910
\end{array}\right.
$$

Figure 17: Map of sampling stations showing area of anomalous tritium for Dec. 1992 - April 1993. 
TRITIUM IN RAIN, APR.-AUG. 1993
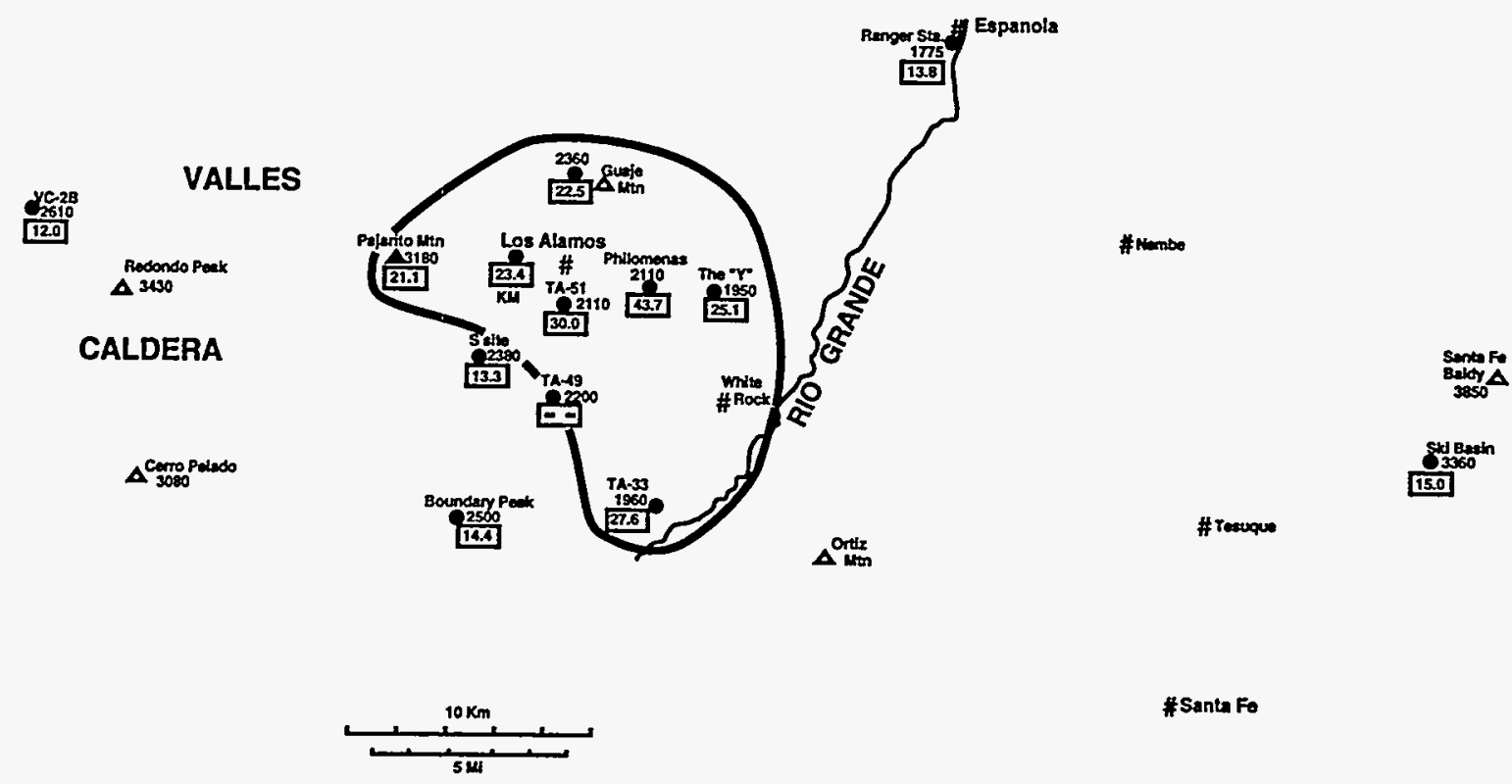

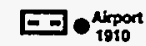

Figure 18: Map of sampling stations showing area of anomalous tritium for April -Aug. 1993.

TRITIUM IN RAIN, AUG.-NOV. 1993
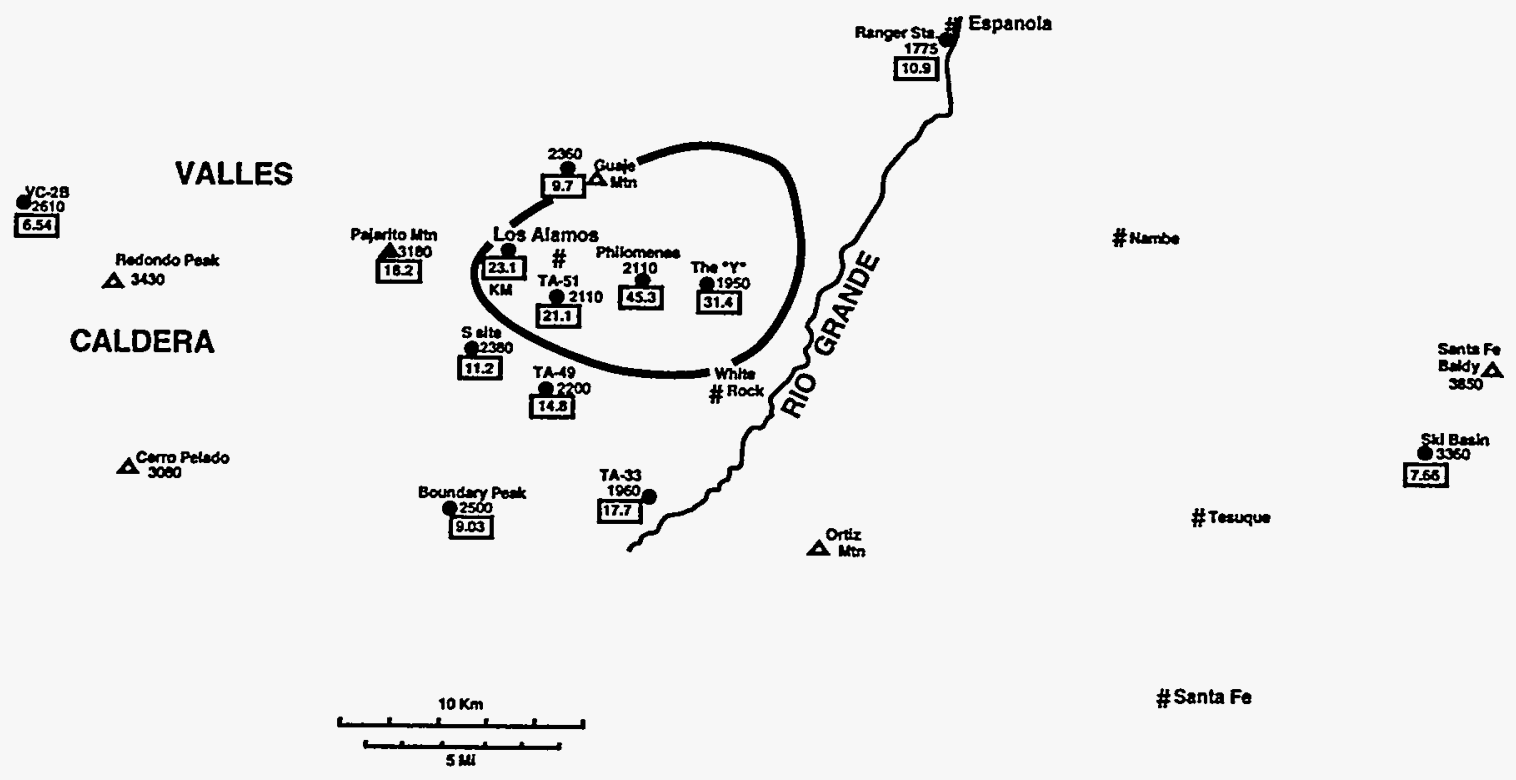

\#Santa Fe

$7007 \bullet_{1910}^{\text {Nhport }}$

Figure 19: Map of sampling stations showing area of anomalous tritium for Aug.-Dec. 1993. 


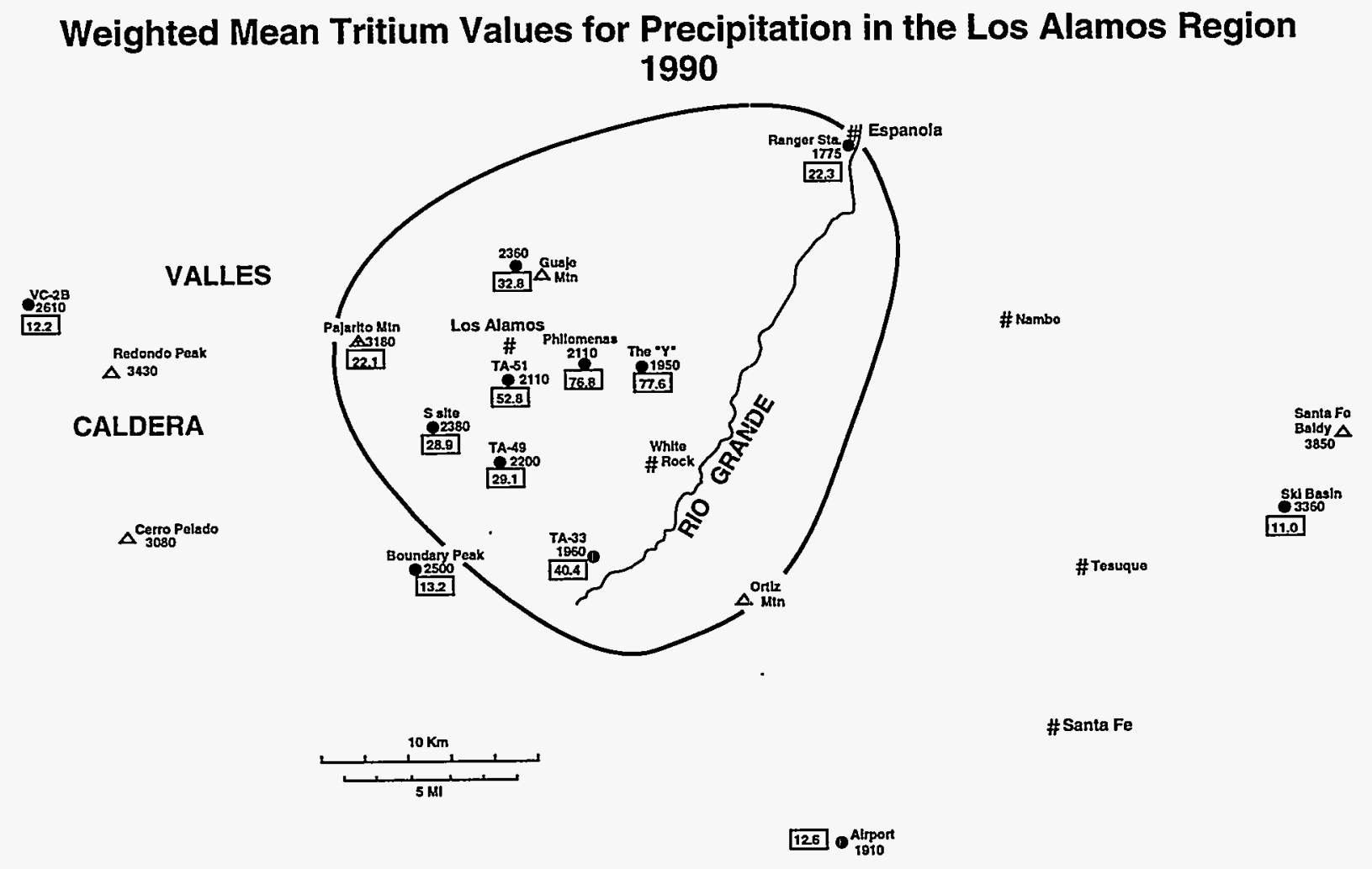

Figure 20: Map of sampling stations using weighted mean tritium values for 1990.
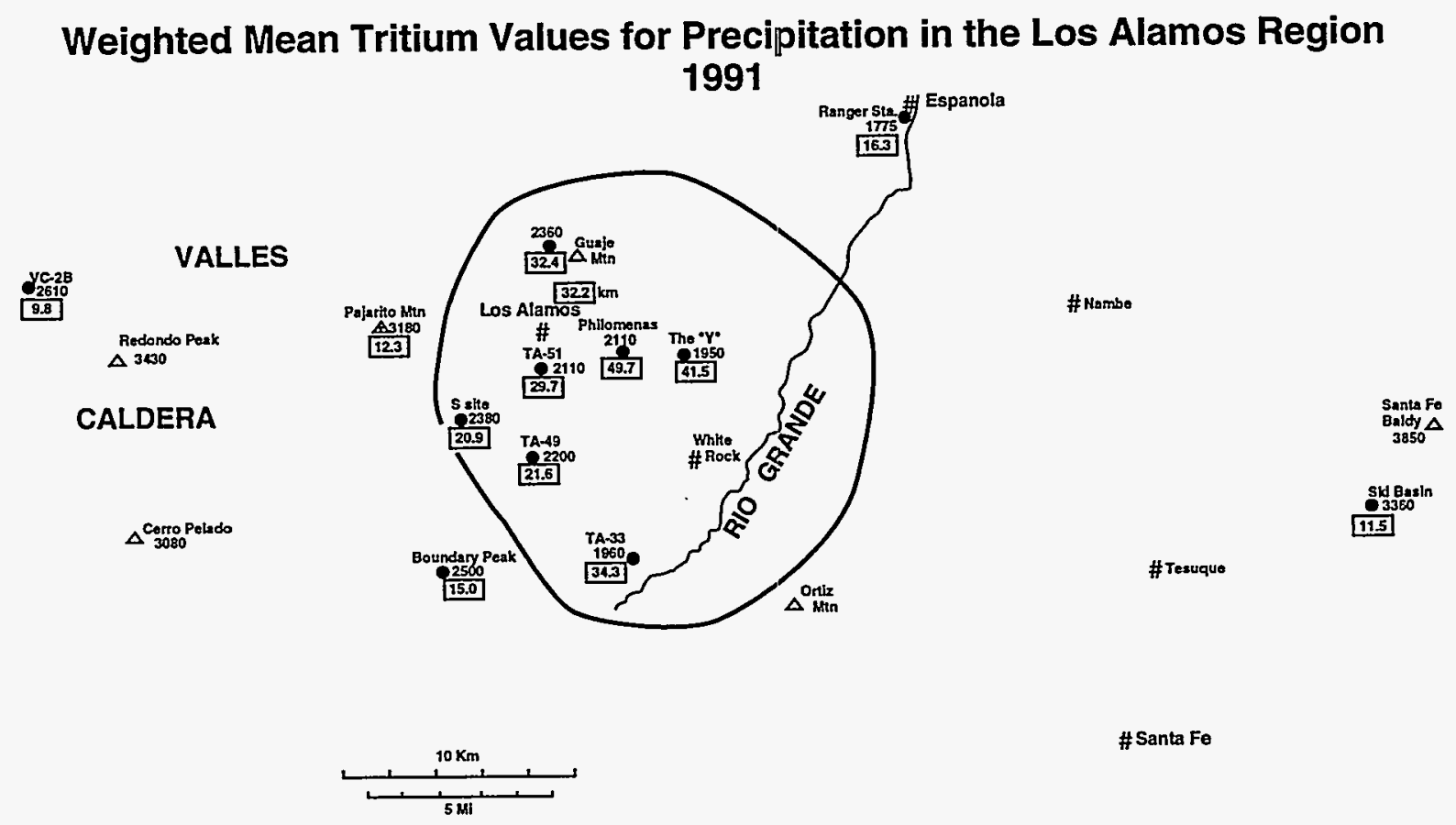

\#Santa $\mathrm{Fe}$

[13.1. A Alport

Figure 21: Map of sampling stations using weighted mean tritium values for 1991. 


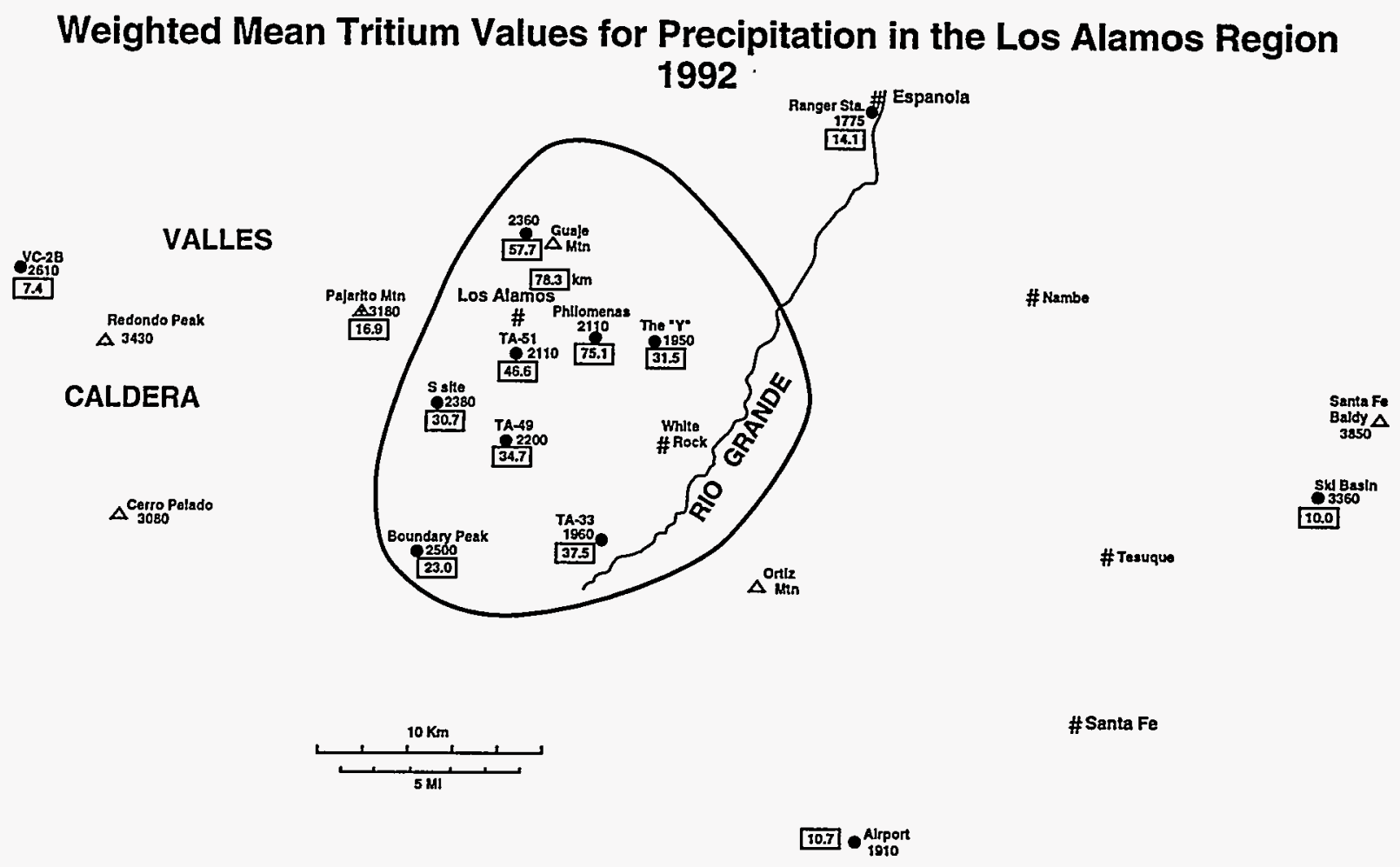

Figure 22: Map of sampling stations using weighted mean tritium values for 1992.

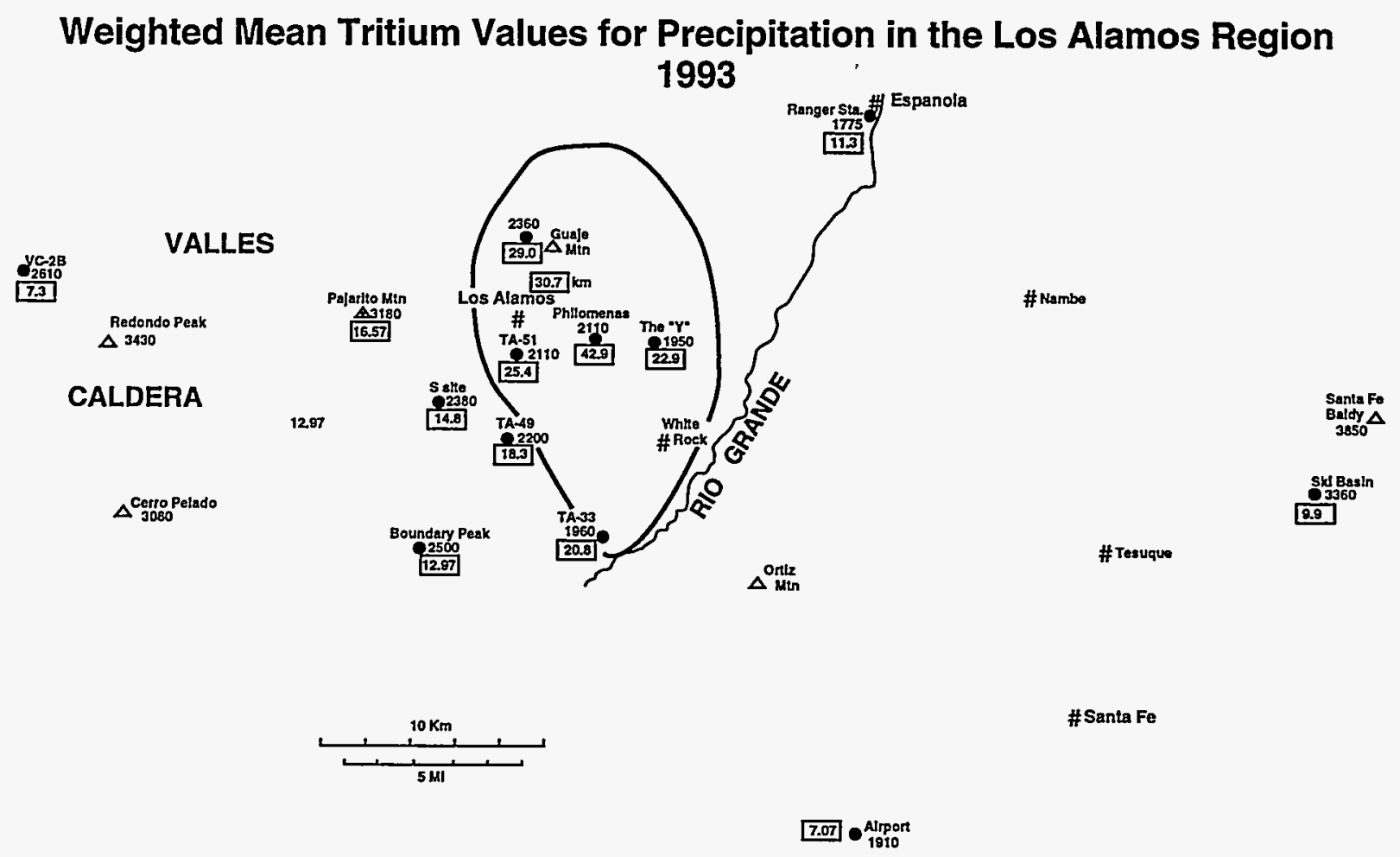

Figure 23: Map of sampling stations using weighted mean tritium values for 1993. 
Sulphur Springs in Valles caldera; Table 4 and Figs. 8-23) and are located outside the influence of the Laboratory. Although tritium values $>20 \mathrm{~T}$.U. are considered anomalous when compared to present background levels in precipitation for this region, all tritium levels measured in this study are less than the Environmental Protection Agency (EPA) threshhold value of 6200 T.U. $\left(20,000 \mathrm{pCi} / \mathrm{liter} \mathrm{H}_{2} \mathrm{O}\right)$ for drinking water.

Weighted Mean Isotope Values: The data presented in this report may be useful to other projects within the Environmental Restoration and Surveillance programs of the Laboratory or for research projects related to groundwater and precipitation. Isotope data for precipitation, particularly tritium data, is usually reported as yearly weighted mean values, $I_{w m}$, in which

$$
\mathrm{I}_{\mathrm{wm}}=\frac{\Sigma(\mathrm{l} \times \mathrm{V})}{\Sigma \mathrm{V}_{\text {tot }}}
$$

where $\mathrm{I}=$ an analysis determined for an isotope parameter at a given sampling point, $\mathrm{V}=$ the volume of water measured at the sampling point where the isotope analysis is determined, and Vtot $=$ the total volume of water measured for the year at the sampling point. The yearly weighted mean values for the deuterium, oxygen-18, and tritium determinations of this study are given in Table 5. Some values calculated for the data at some sampling stations are slightly biased to one collection period relative to another because of missing or contaminated samples as mentioned above.

The stable isotope values of precipitation are quite variable depending on climate, weather patterns, elevation, and latitude (Hoefs, 1973). To obtain a good value for the average composition of precipitation at any sampling point requires several years of continuous data collection; thus, the isotope data generated by this investigation provide a good start on determination of average compositions, reported as composite weighted means 1990 - 1993 (Table 5). When the composite values for the 14 precipitation stations are examined, they show that elevation difference is generally the most important long-term variable controlling stable isotope composition of precipitation in this region. For example, precipitation at the Santa Fe airport (1910 $\mathrm{m}$ ) has composite mean $\mathrm{dD} / \mathrm{d} 180$ values of $-60.7 \%$ and $-8.56 \%$, whereas Santa Fe ski basin $(3360 \mathrm{~m})$ has composite mean values of $-83.2 \%$ and $-12.4 \%$. Higher elevation results in more depleted isotope values if all other variables are held constant. We note, however, that the site with the lowest elevation in this study (Española ranger station) has composite mean stable isotope values of $-76.8 \%$ and $-10.6 \%$, suggesting that other variables have significant effects over relatively short time periods.

Regional tritium variations in groundwaters have been discussed previously by Vuataz and Goff (1986) and Shevenell and Goff (in press). The "pre-bomb" background value of tritium in precipitation for the Los Alamos region was about 6 T.U. At the height of atmospheric nuclear testing, the 1963 tritium level in precipitation reached an average value of 2800 T.U. for the southwestern United States. Because tritium has a short half-life (12.4 yr), the anthropogenic level of tritium in precipitation has decayed rapidly to the present regional value of about $10 \mathrm{~T} . \mathrm{U}$. observed in samples from the three stations located outside of Laboratory influence. For those three stations (and for most stations), there is a general decrease in yearly weighted mean tritium content and, all variables being equal, the weighted mean values should asymptotically approach 6 T.U. as time goes on. Local contamination of present atmospheric tritium by other anthropogenic sources, such as produced by Laboratory activities, will no doubt prolong the life of the semi-circular tritium anomaly observed in the Los Alamos region. Based on our data, the dimensions and magnitude of this anomaly are decreasing with time.

\section{CONCLUSIONS}

The chemistry and stable isotopes values measured in this investigation clearly show that precipitation in the Los Alamos region is relatively normal. However, elevated tritium levels measured in many samples (especially when correlated with the prevailing winds of the region) show that Los Alamos National Laboratory 
TABLE 5. Isotopes in Precipitation in the Los Alamos Region, New Mexico Weighted Mean Averages by Year

\begin{tabular}{|c|c|c|c|c|}
\hline & Del D & Del 180 & Tritium & Comments \\
\hline $\begin{array}{lc}\text { Santa Fe Airport } & 1990 \\
& 1991 \\
& 1992 \\
& 1993 \\
& \\
\text { Composite weighted means 1990-1993 }\end{array}$ & $\begin{array}{l}-44.2 \\
-59.5 \\
-74.4 \\
-59.9 \\
-60.7\end{array}$ & $\begin{array}{r}-4.41 \\
-9.07 \\
-10.59 \\
-9.17 \\
-8.56\end{array}$ & $\begin{array}{r}12.58 \\
13.10 \\
10.66 \\
7.07\end{array}$ & 1 collection in 1993 \\
\hline $\begin{array}{lc}\text { Tech Area } 33 & 1990 \\
& 1991 \\
1992 \\
1993 \\
\text { Composite weighted means 1990-1993 }\end{array}$ & $\begin{array}{l}-64.7 \\
-77.9 \\
-65.0 \\
-80.6 \\
-73.1\end{array}$ & $\begin{array}{r}-9.60 \\
-11.03 \\
-10.57 \\
-11.88 \\
-10.99\end{array}$ & $\begin{array}{l}40.35 \\
34.49 \\
37.64 \\
20.76\end{array}$ & \\
\hline $\begin{array}{l}\text { Philomena's } \\
\qquad \begin{array}{l}1990 \\
1991 \\
1992 \\
1993 \\
\text { Composite weighted means 1990-1993 }\end{array}\end{array}$ & $\begin{array}{l}-69.1 \\
-84.9 \\
-77.2 \\
-81.5 \\
-78.6\end{array}$ & $\begin{array}{r}-9.47 \\
-11.89 \\
-10.82 \\
-11.85 \\
-11.45\end{array}$ & $\begin{array}{l}78.67 \\
49.68 \\
75.11 \\
42.86\end{array}$ & \\
\hline $\begin{array}{l}\text { Pajarito Mtn. } \\
\qquad \begin{array}{l}1990 \\
1991 \\
1992 \\
1993\end{array} \\
\text { Composite weighted means 1990-1993 }\end{array}$ & $\begin{array}{l}-73.5 \\
-66.4 \\
-85.1 \\
-87.7 \\
-77.7\end{array}$ & $\begin{array}{r}-9.80 \\
-10.34 \\
-12.61 \\
-12.75 \\
-11.21\end{array}$ & $\begin{array}{l}22.09 \\
10.96 \\
16.88 \\
16.57\end{array}$ & \\
\hline $\begin{array}{l}\text { S Site } \\
\begin{array}{l}1990 \\
1991 \\
1992 \\
1993 \\
\text { Composite weighted means } 1990-1993\end{array}\end{array}$ & $\begin{array}{l}-75.3 \\
-76.7 \\
-70.1 \\
-82.7 \\
-76.9\end{array}$ & $\begin{array}{l}-11.24 \\
-11.12 \\
-10.26 \\
-12.11 \\
-11.26\end{array}$ & $\begin{array}{l}28.88 \\
20.87 \\
30.72 \\
14.85\end{array}$ & \\
\hline $\begin{array}{l}\text { White Rock } Y \\
\qquad \begin{array}{l}1990 \\
1991 \\
1992 \\
1993\end{array} \\
\text { Composite weighted means 1990-1993 }\end{array}$ & $\begin{array}{l}-64.3 \\
-80.6 \\
-80.2 \\
-81.7 \\
-74.5\end{array}$ & $\begin{array}{r}-7.81 \\
-11.07 \\
-11.48 \\
-11.73 \\
-10.46\end{array}$ & $\begin{array}{l}77.64 \\
25.93 \\
31.54 \\
22.86\end{array}$ & \\
\hline $\begin{array}{c}\text { Espanola Ranger Station } \\
1990 \\
1991 \\
1992 \\
1993 \\
\text { Composite weighted means 1990-1993 }\end{array}$ & $\begin{array}{l}-70.5 \\
-74.2 \\
-83.6 \\
-76.4 \\
-76.8\end{array}$ & $\begin{array}{r}-9.98 \\
-9.71 \\
-11.65 \\
-10.86 \\
-10.59\end{array}$ & $\begin{array}{l}22.30 \\
16.26 \\
14.09 \\
11.29\end{array}$ & \\
\hline $\begin{array}{r}\text { Boundary Peak Area } \\
1990 \\
1991 \\
1992 \\
1993 \\
\end{array}$ & $\begin{array}{l}-84.1 \\
-69.2 \\
-63.3 \\
-88.1 \\
-77.9\end{array}$ & $\begin{array}{r}-9.15 \\
-8.38 \\
-9.26 \\
-12.93 \\
-10.19\end{array}$ & $\begin{array}{l}13.61 \\
15.91 \\
23.00 \\
12.97\end{array}$ & $\begin{array}{l}2 \text { collections in } 1990 \\
1 \text { collection in } 1991 \\
1 \text { collection in } 1992 \\
\text { Last collection of } 93 \\
\text { was collected } 2 / 94\end{array}$ \\
\hline
\end{tabular}




\begin{tabular}{|c|c|c|c|c|}
\hline & Del D & Del 180 & Tritium & Comments \\
\hline $\begin{array}{ll}\text { West of Guaje Mtn. } & \\
1990 \\
1991 \\
1992 \\
1993 \\
\text { Composite weighted means 1990-1993 }\end{array}$ & $\begin{array}{l}-63.9 \\
-75.8 \\
-85.4 \\
-74.5 \\
-74.9\end{array}$ & $\begin{array}{r}-9.82 \\
-10.90 \\
-12.56 \\
-10.39 \\
-11.01\end{array}$ & $\begin{array}{l}33.23 \\
32.36 \\
57.72 \\
29.01\end{array}$ & 2 collections in 1990 \\
\hline $\begin{array}{ll}\text { Santa Fe Ski Basin } & \\
& 1990 \\
1991 \\
1992 \\
1993 \\
\text { Composite weighted means 1990-1993 }\end{array}$ & $\begin{array}{l}-64.1 \\
-87.1 \\
-85.6 \\
-84.6 \\
-83.2\end{array}$ & $\begin{array}{r}-9.69 \\
11.79 \\
-13.21 \\
-12.05 \\
-12.35\end{array}$ & $\begin{array}{r}11.82 \\
11.50 \\
10.82 \\
9.93\end{array}$ & 2 collections in 1990 \\
\hline $\begin{array}{l}\text { VC-2B Sulphur Springs } \\
1990 \\
1991 \\
1992 \\
1993 \\
\text { Composite weighted means 1990-1993 }\end{array}$ & $\begin{array}{r}-66.1 \\
-87.4 \\
-109.1 \\
-82.1 \\
-87.7\end{array}$ & $\begin{array}{r}-9.48 \\
-12.44 \\
-15.69 \\
-9.76 \\
-12.85\end{array}$ & $\begin{array}{l}7.05 \\
9.77 \\
7.67 \\
7.31\end{array}$ & 2 collections in 1992 \\
\hline $\begin{array}{ll}\text { Tech Area } 49 & 1990 \\
& 1991 \\
1992 \\
1993 \\
\text { Composite weighted means 1990-1993 }\end{array}$ & $\begin{array}{l}-55.9 \\
-77.1 \\
-67.1 \\
-86.9 \\
-74.0\end{array}$ & $\begin{array}{r}-7.62 \\
-10.85 \\
-9.84 \\
-12.74 \\
-10.59\end{array}$ & $\begin{array}{l}29.46 \\
21.57 \\
34.66 \\
19.38\end{array}$ & $\begin{array}{l}2 \text { collections in } 1990 \\
2 \text { collections in } 1993\end{array}$ \\
\hline $\begin{array}{l}1990 \\
1991 \\
1992 \\
1993\end{array}$ & $\begin{array}{l}-61.9 \\
-78.9 \\
-68.8 \\
-82.2\end{array}$ & $\begin{array}{r}-9.42 \\
-11.02 \\
-10.22 \\
-11.87\end{array}$ & $\begin{array}{l}53.51 \\
29.69 \\
46.56\end{array}$ & 2 collections in 1990 \\
\hline Composite weighted means 1990-1993 & -75.3 & -11.03 & & \\
\hline $\begin{array}{lc}\text { K.M. House } & 1991 \\
1992 \\
1993\end{array}$ & $\begin{array}{l}-72.9 \\
-76.4 \\
-75.5 \\
-74.8\end{array}$ & $\begin{array}{l}-10.81 \\
-10.85 \\
-10.91 \\
-10.82\end{array}$ & $\begin{array}{l}32.60 \\
78.30 \\
30.70\end{array}$ & 2 collections in 1991 \\
\hline
\end{tabular}

is releasing tritium to the atmosphere. This anomalous tritium is being added to background levels of tritium present in the atmosphere and the combined tritium is precipitated locally. The dimensions and magnitude of the tritium anomaly are apparently decreasing with time. Fortunately, the amount of tritium in regional precipitation is very low when compared to the EPA threshhold value of drinking water (about 6200 T.U.).

\section{ACKNOWLEDGMENTS}

The authors gratefully appreciate the encouragement and support of Micheline Devaurs, David Broxton, and Jamie Gardner (Los Alamos National Laboratory) who helped get this project off the ground and kept it alive. Many thanks to Kim Manley (Los Alamos), John Corbin (Sulphur Springs), and authorities at Santa Fe ski basin, Santa Fe airport, Pajarito Mountain ski hill, and Española ranger station for protecting our precipitation collectors during the investigation. The draft manuscript was reviewed by James A. Stimac (Los Alamos National Laboratory). Financial support came primarily from the Environmental Restoration Program of Los Alamos National Laboratory. 


\section{REFERENCES}

Adams, A.I., and Goff, F., 1991, "A Regional Study of Stable Isotopes and Tritium in Precipitation in the Los Alamos Region of New Mexico," Geol. Soc. Amer., Abs. w/Programs, v. 23(5), p. 115.

Adams, A.l., and Goff, F., 1994, "Environmental Surveillance at Los Alamos during 1992," Los Alamos National Laboratory Rept. LA-12764-ENV, p. IV83 - IV86.

Blake, W.D., Goff, F., Adams, A.I., and Counce D., in press, "Environmental Geochemistry for Surface and Subsurface Waters in the Pajarito Plateau and Outlying Areas, New Mexico," Los Alamos National Laboratory Rept., MS-series.

Craig, H., 1961, "Isotopic Variations in Meteoric Waters," Science, v. 133, p. 1702-1703.

Drever, J.I., 1982, "The Geochemistry of Natural Waters," Prentice Hall, Inc., N.J., 388 pp.

Goff, F, and Sayer, S., 1980, "A Geothermal Investigation of Spring and Well Waters of the Los Alamos Region, New Mexico," Los Alamos Scientific Laboratory Rept. LA-8326-MS, 21 pp.

Goff, F., Gardner, J.N., Vidale, R., and Charles, R., 1985, “Geochemistry and Isotopes of Fluids from Sulphur Springs, Valles Caldera, New Mexico," Jour. Volcanol. Geotherm. Res., v. 23, p. 273-297.

Hoefs, J., 1973, "Stable Isotope Geochemistry," Springer-Verlag, N.Y., 140 pp.

Junge, C.E., and Werby, R.T., 1958, "The Concentration of Chloride, Sodium, Potassium, Calcium, and Sulfate in Rain Water over the United States," Jour. Meteorol., v. 15, p. 417-425.

Shevenell, L., Goff, F., Vuataz, F., Trujillo, P.E., Counce, D., Janik, C.J., and Evans, W., 1987, "Hydrogeochemical Data for Thermal and Nonthermal Waters and Gases of the Valles CalderaSouthern Jemez Mountains Region, New Mexico," Los Alamos National Laboratory Rept. LA-10923OBES, $100 \mathrm{pp}$.

Shevenell, L., and Goff, F., in press, "The Use of Tritium in Groundwater to Determine Fluid Mean Residence Times of Valles Caldera Hydrothermal Fluids, New Mexico, USA," Jour. Volcanol. Geotherm. Res.

Trainer, F.W., 1974, "Ground-water in the Southern Part of the Jemez Mountains Volcanic Region, New Mexico," New Mexico Geol. Soc. Guidebook, 25th Field Conf., p. 337-345.

Trujillo, P.E., Counce, D., Grigsby, C.O., Goff, F., and Shevenell, L., 1987, "Chemical Analysis and Sampling Techniques for Geothermal Fluids and Gases at the Fenton Hill Laboratory," Los Alamos National Laboratory Rept. LA-11006-MS, 84 pp.

White, A.F., Peterson, M.L., Wollenberg, H., and Flexser, S., 1990, "Sources and Fractionation Processes Influencing the Isotopic Distribution of $\mathrm{H}, \mathrm{O}$, and $\mathrm{C}$ in the Long Valley Hydrothermal System, California, U.S.A.," Applied Geochem., v. 5, p. 571-585.

Vuataz, F.-D., and Goff, F., 1986, "Isotope Geochemistry of Thermal and Nonthermal Waters in the Valles Caldera, Jemez Mountains, Northern New Mexico," Jour. Geophys. Res., v. 91(B2), p. 1835-1853. 